ANL-99/20

ARGONNE NATIONAL LABORATORY
9700 South Cass Avenue
Argonne, Illinois 60439

\title{
PRACTICAL SUPERCONDUCTOR DEVELOPMENT FOR ELECTRICAL POWER APPLICATIONS \\ ANNUAL REPORT FOR FY 1999
}

U. Balachandran

Contributors:

T. R. Askew

Y. S. Cha

K. C. Goretta

K. E. Gray ${ }^{\dagger}$

J. R. Hull

S. E. Dorris

J. T. Dusek

J. E. Emerson

M. T. Lanagan

M. Lelovic

V. A. Maroni ${ }^{\dagger \dagger}$

R. L. McDaniel

N. Merchant ${ }^{\dagger \dagger}$

D. J. Miller ${ }^{\dagger}$

J.-H. Park

J. J. Picciolo

B. L. Fisher

J. P. Singh

\section{Energy Technology Division}

October 1999

Work supported by

U.S. DEPARTMENT OF ENERGY

Office of Energy Efficiency and Renewable Energy 


\title{
PRACTICAL SUPERCONDUCTOR DEVELOPMENT FOR ELECTRICAL POWER APPLICATIONS
}

\author{
ANNUAL REPORT FOR FY 1999
}

\begin{abstract}
Development of useful high- $\mathrm{T}_{\mathrm{c}}$ superconductors requires synthesis of superconducting compounds; fabrication of wires, tapes, and films from these compounds; production of composite structures that incorporate stabilizers or insulators; and design and testing of efficient components. This report describes FY 1998 technical progress of research and development efforts aimed at producing superconducting components in the $\mathrm{Bi}-(\mathrm{Pb})-\mathrm{Sr}-\mathrm{Ca}-\mathrm{Cu}$ and $\mathrm{Y}-\mathrm{Ba}-\mathrm{Cu}$ oxide systems. Topics discussed are synthesis and heat treatment of high- $\mathrm{T}_{\mathrm{C}}$ superconductors, formation of monolithic and composite conductors, characterization of structures and superconducting and mechanical properties, and fabrication and testing of prototype components. Collaborations with industry and academia are documented.
\end{abstract}

\section{Introduction}

The superconductor program at Argonne National Laboratory (ANL) includes bulk and film processing directed toward improving the properties of high- $\mathrm{T}_{\mathrm{c}}$ superconductors (HTSs), developing processing methods for production of commercial conductors, and fabricating and testing prototype conductors. Cooperative relationships with industrial and academic partners are integral to this program. The principal objective of the ANL program is to develop methods to fabricate and use structurally reliable HTSs for generating, transmitting, and storing electrical energy. Work is now focused on superconductors based on bismuth-lead-strontium-calcium-copper oxide and yttrium-barium-copper oxide.

There are several requirements for composite and monolithic conductors in the form of wires, tapes, films, or other shapes. For most applications, the conductors must be capable of carrying large currents in the presence of large magnetic fields and must be strong, flexible, and chemically and cryogenically stable. Potential applications for such conductors include transmission lines, motors, generators, transformers, magnetic energy storage devices, and electronics. The principal impediments to the use of bulk HTSs are low critical current $\left(\mathrm{I}_{\mathrm{C}}\right)$ and critical current density $\left(\mathrm{J}_{\mathrm{C}}\right)$ in large applied magnetic fields, relatively poor mechanical properties, and difficulty in fabricating very long lengths with uniform properties. Processing methods for the improvement of all have been and continue to be developed. The goals of fabrication include promoting high conductivity by obtaining phase-pure final materials with favorable grain alignment, maximizing flux pinning through microstructural control, and increasing flexibility and reliability by minimizing microstructural flaws and optimizing the strength and toughness of each material. 
This report reviews the technical progress and status of (a) $\mathrm{Bi}$-based high- $\mathrm{T}_{\mathrm{c}}$ wires and tapes, especially those that contain $(\mathrm{Bi}, \mathrm{Pb})_{2} \mathrm{Sr}_{2} \mathrm{Ca}_{2} \mathrm{Cu}_{3} \mathrm{O}_{\mathrm{x}}(\mathrm{Bi}-2223)$; (b) $\mathrm{YBa}_{2} \mathrm{Cu}_{3} \mathrm{O}_{\mathrm{x}}$ (Y-123) superconductors in tape, film, and bulk forms; (c) study of microstructures and fundamental properties; and (d) fabrication and testing of prototype superconducting devices.

\section{Technical Progress in 1998-1999}

\section{1 $\mathrm{Bi}-\mathrm{Pb}-\mathrm{Sr}-\mathrm{Ca}-\mathrm{Cu}-\mathrm{O}$ Superconductors}

\subsubsection{Phase Development}

Bi-2223 Phase Stability. As part of our continuing effort to analyze and elucidate how process parameters, such as temperature, oxygen pressure $\left(\mathrm{pO}_{2}\right)$, and composition, influence Bi-2223 phase formation and microstructure in the context of powder-in-tube processing, we have conducted a thorough assessment of existing data that define the phase stability of Bi-2223. Phase stability data for the Bi-2223 system derived from equilibration and electromotive-force studies carried out by numerous groups of investigators [1-8] were analyzed. The results of this assessment, coupled with additional more recent data from our laboratory, provide a means for estimating the stability of $\mathrm{Bi}-2223$ at $650-870^{\circ} \mathrm{C}$ for $\mathrm{pO}_{2}$ of $10^{-5}-1.0 \mathrm{~atm}$. Table 1 contains a list of the stoichiometries of the $\mathrm{Bi}-2223$ precursors employed in the studies cited in this report. The values in Table 1 show that the compositional variations from study to study are generally small, thus validating the comparisons made.

The plots in Figs. 1 and 2 contain results from the eight studies listed. Figure 1 emphasizes how relevant data from selected equilibration measurements of Kusano et al. [1], Moon et al. [2], and Majewski et al. [3] compare to the more comprehensive equilibration study by Zhu and Nicholsen [4], and the electromotive force (EMF) experiments of Tetenbaum et al. [5] and MacManus-Driscoll et al. [6]. These comparisons manifest themselves in the $\mathrm{pO}_{2}$ range of 0.05-0.21 atm, with a corresponding temperature range of $800-860^{\circ} \mathrm{C}-\mathrm{a}$ domain of $\mathrm{pO}_{2}$ and temperature that encompasses the commercially pertinent production zone for processing of tapes and wires. This zone is indicated in Figs. 1 and 2 by the triangular sliver between the horizontal lines marking the $7.5 \% \mathrm{O}_{2}$ and $21 \% \mathrm{O}_{2}$ pressure values. Figure 2 focuses attention on the equilibration data of Daumling et al. [7] in comparison with the emf data of Tetenbaum et al. [5] and MacManus-Driscoll et al. [6], particularly at $\mathrm{pO}_{2}<2 \% \quad(<0.02 \mathrm{~atm})$, noting the better agreement with the Tetenbaum et al. Data that indicate that the low $\mathrm{pO}_{2}$ decomposition boundary of $\mathrm{Bi}-2223$ are nearly asymptotic to the $\mathrm{CuO} / \mathrm{Cu}_{2} \mathrm{O}$ stability curve.

Another important observation from the work of Daumling et al. [7] and recent work in our laboratory (Murphy and Dorris [8]) addressing the stability of silver-sheathed Bi-2223, is that the presence of silver shifts the location of the high-temperature boundary to lower temperatures, particularly at $\mathrm{pO}_{2}$ values in the production zone. We are continuing this study to determine the extent to which filament dimensions (insofar as they affect the average proximity of $\mathrm{Bi}-2223$ to silver) influence the stability of Bi-2223. 
Table 1. Stoichiometries of $\mathrm{Bi}-2223$ used in stability studies

\begin{tabular}{ll}
\hline \multicolumn{1}{c}{ Reference } & \multicolumn{1}{c}{$\mathrm{Bi}-2223$ Stoichiometry } \\
\hline 1. Kusano et al. & $\mathrm{Bi}_{1.8} \mathrm{~Pb}_{0.2} \mathrm{Sr}_{2.0} \mathrm{Ca}_{2.0} \mathrm{Cu}_{3.2} \mathrm{O}_{\mathrm{x}}$ \\
2. Moon et al. & $\mathrm{Bi}_{1.8} \mathrm{~Pb}_{0.3} \mathrm{Sr}_{1.9} \mathrm{Ca}_{2.0} \mathrm{Cu}_{3.0} \mathrm{O}_{\mathrm{x}}$ \\
3. Majewski et al. & $\mathrm{Pb}^{\circ} 0.3$ \\
4. Zhu and Nicholsen & $\mathrm{Bi}_{1.8} \mathrm{~Pb}_{0.3} \mathrm{Sr}_{1.9} \mathrm{Ca}_{2.0} \mathrm{Cu}_{3.1} \mathrm{O}_{\mathrm{x}}$ \\
5. Tetenbaum et al. & $\mathrm{Bi}_{1.7} \mathrm{~Pb}_{0.3} \mathrm{Sr}_{2.0} \mathrm{Ca}_{2.2} \mathrm{Cu}_{3.0} \mathrm{O}_{\mathrm{x}}$ \\
6. MacManus-Driscoll et al. & $\mathrm{Bi}_{1.8} \mathrm{~Pb}_{0.3} \mathrm{Sr}_{2.0} \mathrm{Ca}_{2.2} \mathrm{Cu}_{3.0} \mathrm{O}_{\mathrm{x}}$ \\
7. Daumling et al. & $\mathrm{Bi}_{1.7} \mathrm{~Pb}_{0.3} \mathrm{Sr}_{1.8} \mathrm{Ca}_{2.0} \mathrm{Cu}_{3.1} \mathrm{O}_{\mathrm{x}}$ \\
8. Murphy and Dorris & $\mathrm{Bi}_{1.7} \mathrm{~Pb}_{0.3} \mathrm{Sr}_{2.0} \mathrm{Ca}_{2.2} \mathrm{Cu}_{3.0} \mathrm{O}_{\mathrm{x}}$ \\
\hline
\end{tabular}

Key findings of our assessment are that (a) the Bi-2223 phase stability boundaries are reasonably well known based on data several sources for bulk material [the data for $\mathrm{Ag} / \mathrm{Bi}-2223$ are less comprehensive]; (b) generally good agreement exists for $\mathrm{pO}_{2}=0.075-0.021 \mathrm{~atm}$ at $810-850^{\circ} \mathrm{C}$; (c) proximity to $\mathrm{Ag}$ (as mentioned above) lowers the upper-temperature stability boundary; and (d) discrepancies in the data for bulk Bi-2223 exist at low $\mathrm{pO}_{2}\left(<10^{-2} \mathrm{~atm}\right)$ and low temperature $\left(<700^{\circ} \mathrm{C}\right)$. The extent to which oxygen and/or cation diffusion limits the measurement capability at temperatures below $800^{\circ} \mathrm{C}$ is a topic of consideration in our ongoing effort to maximize the performance of $\mathrm{Ag} / \mathrm{Bi}-2223$ composite conductors.

Decomposition/Re-formation of $\mathrm{Bi}-2223$. Working in conjunction with American Superconductor Corporation (ASC) and the ASC-led Wire Development Group, we have completed an investigation of the phase and microstructural stability of Bi-2223 in a Ag-sheathed monofilament composite tape fabricated from fine-grained, fully transformed Bi-2223 powder. The purpose of this study was to explore the prospects of growing textured, large-grain-size $\mathrm{Bi}-2223$ from fine-grained preconverted precursors. Prior studies of $\mathrm{Bi}-2223$ decomposition/re-formation have shown that treatments such as re-formation can strongly influence microstructure, phase chemistry, and performance of $\mathrm{Ag} / \mathrm{Bi}-2223$ composites. However, the starting-phase assemblage in the previous tapes was Bi-2212 plus secondary phases (with overall Bi-2223 stoichiometry), and formation of Bi-2223 was carried out in the Ag sheath, whereas in this work, the starting-phase assemblage was $\geq 95 \%$ fine-grained $\mathrm{Bi}-2223$.

The monofilamentary composite tapes employed in this research were made by standard drawing and rolling methods. The precursor powder had a nominal composition of $\mathrm{Bi}_{1.7} \mathrm{~Pb}_{0.3} \mathrm{Sr}_{1.9} \mathrm{Ca}_{2.0} \mathrm{Cu}_{3.0} \mathrm{O}_{\mathrm{x}}$ and was composed of fine-grained $\mathrm{Bi}-2223$ mixed with small amounts of residual $\mathrm{Bi}_{2} \mathrm{Sr}_{2} \mathrm{CaCu}_{2} \mathrm{O}_{\mathrm{x}}(\mathrm{Bi}-2212)$ and alkaline earth cuprates. Samples were either heated at $10^{\circ} \mathrm{C} / \mathrm{min}$ to $800^{\circ} \mathrm{C}$ and $2^{\circ} \mathrm{C} / \mathrm{min}$ to $825^{\circ} \mathrm{C}$ or immersion-heated at $100^{\circ} \mathrm{C} / \mathrm{min}$ to an initial temperature of $825^{\circ} \mathrm{C}$ under a $\mathrm{pO}_{2}$ of either 0.075 or 0.046 atm. After an initial $3000-\mathrm{min}$ hold at $825^{\circ} \mathrm{C}$ in 0.075 atm $\mathrm{O}_{2}$, temperature excursions, either downward to $780^{\circ} \mathrm{C}$ for $30 \mathrm{~min}$ or upward to $855^{\circ} \mathrm{C}$ for $20 \mathrm{~min}$, were programmed into the annealing cycle. Oxygen pressure variations to $0.002,0.02$, and $0.046 \mathrm{~atm}$ during 


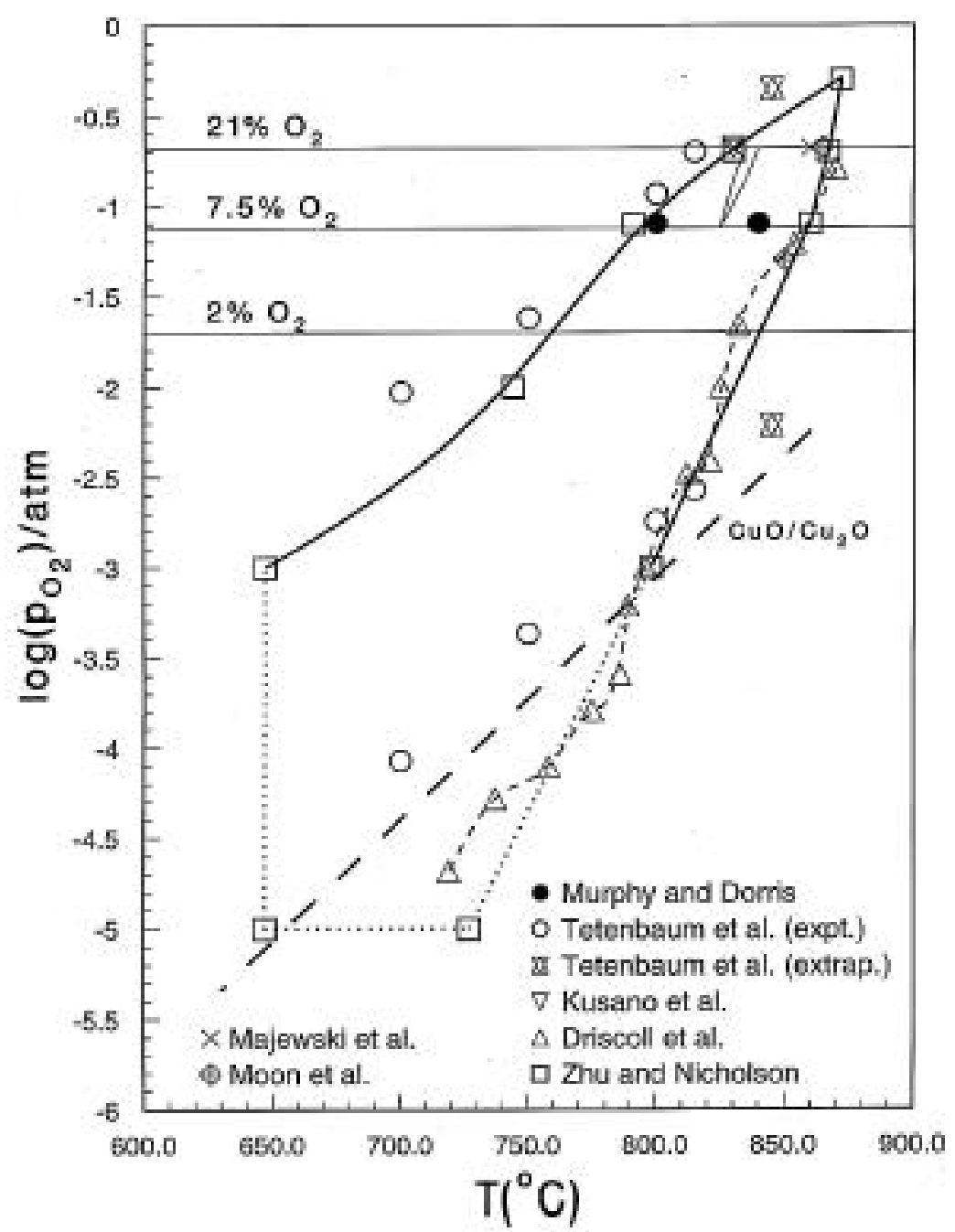

Fig. 1. Phase stability data for Bi-2223 from various studies [1-6,8], emphasizing results that fall near the commercial "production zone" for silver-sheathed $\mathrm{Bi}-2223$ composite conductor (i.e., triangular sliver between $7.5 \% \mathrm{O}_{2}$ and $21 \% \mathrm{O}_{2}$ ).

annealing at $825^{\circ} \mathrm{C}$ were also studied. Samples were either quenched or furnace-cooled at the end of each heat treatment. They were characterized by scanning electron microscopy (SEM), energy-dispersive X-ray analysis (EDS), and X-ray diffraction (XRD).

Two of the heat treatment cycles used in the decomposition/re-formation studies reported here are shown in Fig. 3. XRD measurements on samples annealed under the cycle depicted in Fig. 3a, but quenched immediately on reaching $825^{\circ} \mathrm{C}$ in $0.075 \mathrm{~atm} \mathrm{O}_{2}$, revealed that as much as $50 \%$ of the $\mathrm{Bi}-2223$ phase decomposed to $\mathrm{Bi}-2212$ and secondary phases during heating to $825^{\circ} \mathrm{C}$. Similar decomposition of the $\mathrm{Bi}-2223$ phase was also observed when the samples were immersion-heated. However, in subsequent studies, we 


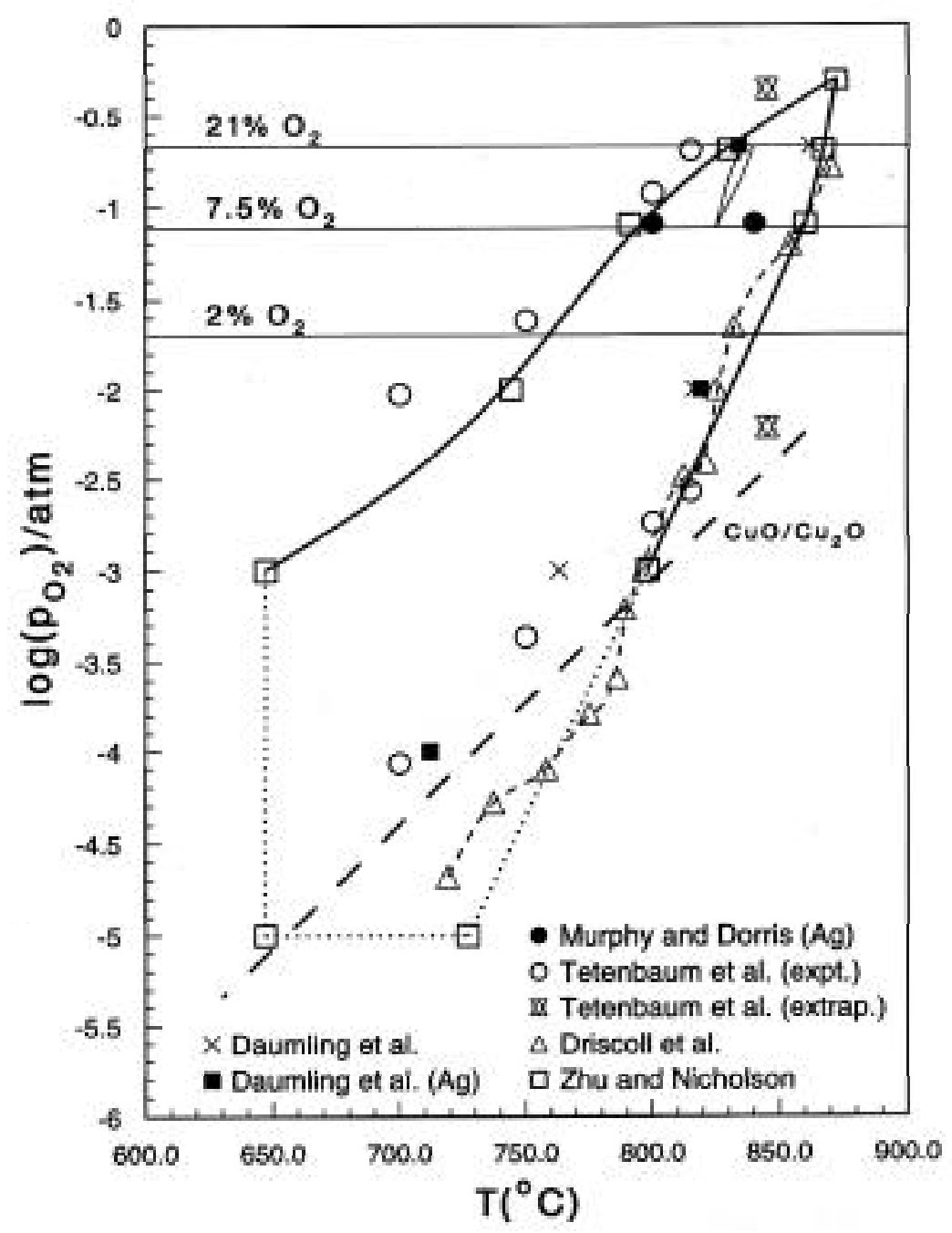

Fig. 2. Phase stability data for $\mathrm{Bi}-2223$ from various studies [4-8], emphasizing results that fall below $2 \% \mathrm{O}_{2}(<0.02 \mathrm{~atm})$.

found that this decomposition could be fully arrested by ramp-heating the sample to $825^{\circ} \mathrm{C}$ under a $\mathrm{pO}_{2}$ of $0.002 \mathrm{~atm}$. This result occurs mainly because the phase stability boundaries of $\mathrm{Bi}-2223$ are crossed during heating in 0.075 atm $\mathrm{O}_{2}$, but are not crossed in the lower $\mathrm{pO}_{2}$ of $0.002 \mathrm{~atm}$.

Figure 4 is an SEM photomicrograph of the transverse cross section of a sample annealed for $6000 \mathrm{~min}$ in $0.075 \mathrm{~atm} \mathrm{O}_{2}$ at $825^{\circ} \mathrm{C}$ and furnace-cooled. Layered-phase grain growth is essentially nonexistent and transverse cracks appear with regularity. This, we believe, is because during normal processing of a tape with $\mathrm{Bi}-2223$ as the initial major phase, little or no liquid phase is formed during heat treatment to facilitate grain growth. Also, cracks that may have formed during the deformation processing before the annealing cannot be healed because of the persistent absence of liquid phase. 

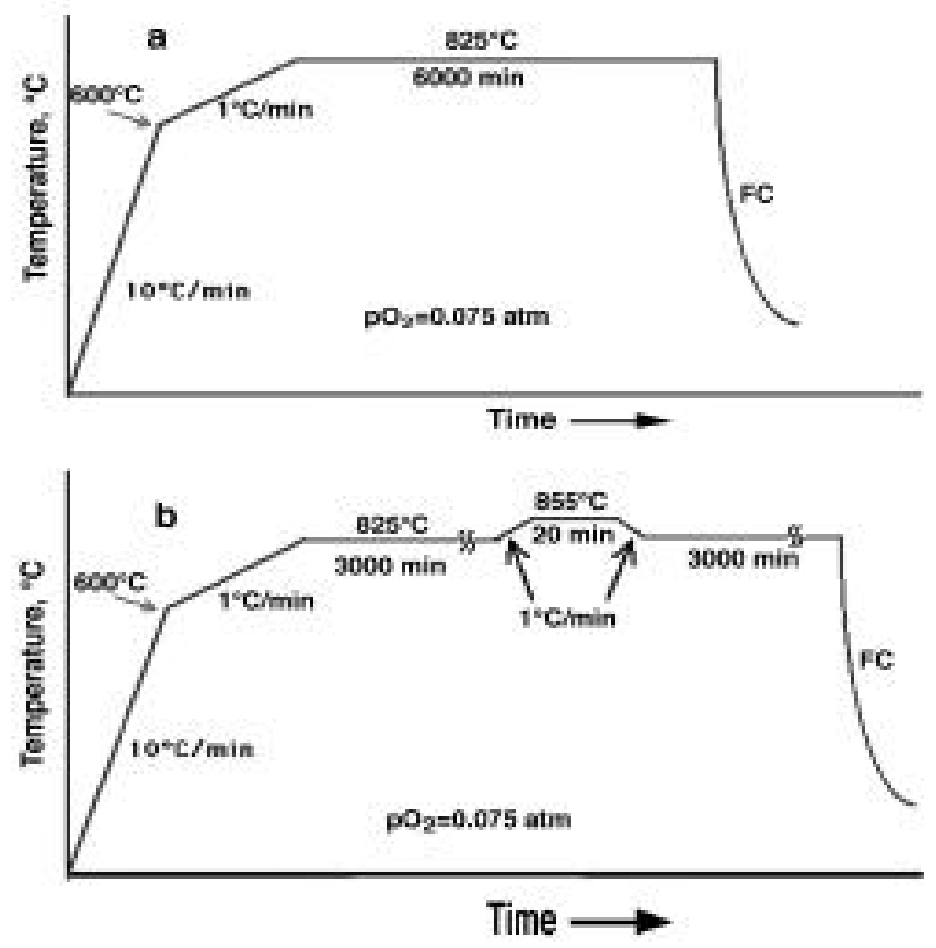

Fig. 3. Heat treatment cycles for $\mathrm{Ag} / \mathrm{Bi}-2223$ decomposition/ re-formation tests: (a) blank run and (b) partial-melt simulation. $\mathrm{FC}=$ furnace cooled.

An SEM photomicrograph of a sample annealed by the cycle shown in Fig. 3b, which features a $20-$ min excursion to $855^{\circ} \mathrm{C}$ followed by a $3000-$ min recovery treatment at $825^{\circ} \mathrm{C}$, is shown in Fig. 5. Layered-phase grain growth occurred under these conditions. No cracks are visible in the sample, indicating that cracks that formed during deformation processing were healed, most probably because of the presence of a liquid phase. XRD data showed that the sample is $\approx 80 \% \mathrm{Bi}-2223,16 \% \mathrm{Bi}-2212$, and $4 \% \mathrm{Bi}_{2} \mathrm{Sr}_{2} \mathrm{CuO}_{\mathrm{x}}(\mathrm{Bi}-2201)$. It is not clear that annealing the sample for longer than $3000 \mathrm{~min}$ after the high-temperature excursion will necessarily produce complete re-formation of the Bi-2223. Alternatively, the excursion temperature and/or time may have to be shortened to limit the decomposition of $\mathrm{Bi}-2223$. It is noteworthy that the nonsuperconducting secondary phases are quite small after this decomposition/re-formation treatment.

The transmission electron microscopy (TEM) photomicrograph shown in Fig. 6 was obtained from a sample that was subjected to the cycle shown in Fig. $3 \mathrm{~b}$. This photomicrograph highlights a unique $z$ effect of the decomposition/re-formation treatment, i.e., the occurrence near the $\mathrm{Ag} /$ superconductor interface of large adjacent Bi-2223/Bi-2212 grain colonies that exhibit (001) orientation.

Our efforts during this study to investigate reduced-temperature dips in the annealing cycle, e.g., $825^{\circ} \mathrm{C}$ for $3000 \mathrm{~min}, 780^{\circ} \mathrm{C}$ for $30 \mathrm{~min}, 825^{\circ} \mathrm{C}$ for $3000 \mathrm{~min}$ in $0.075 \mathrm{~atm} \mathrm{O}_{2}$, produced no discernible changes in the $\mathrm{Bi}-2223$ microstructure. Attempts to use longer 


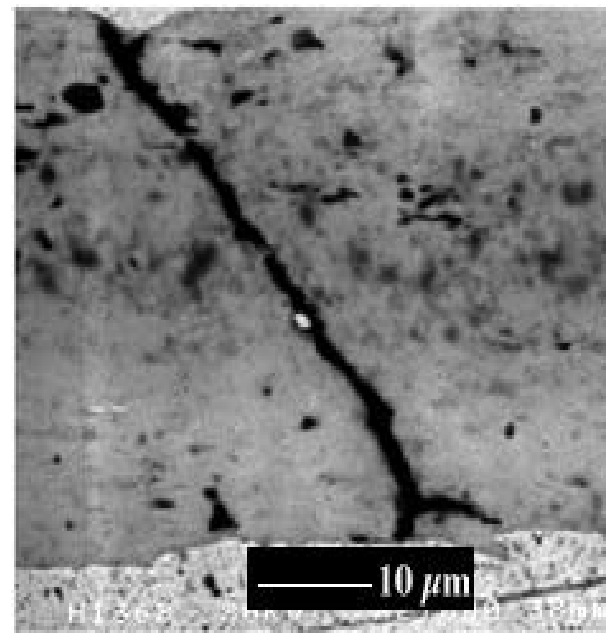

Fig. 4.

SEM photomicrograph in backscatter mode of transverse section of monofilament sample annealed for $6000 \mathrm{~min}$ at $825^{\circ} \mathrm{C}$ in $0.075 \mathrm{~atm} \mathrm{O}_{2}$.

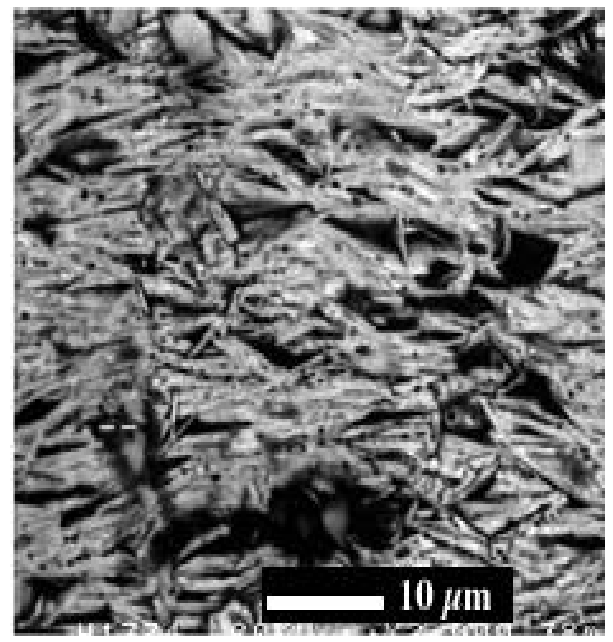

Fig. 5.

SEM photomicrograph in backscatter mode of transverse section of sample annealed by cycle shown in Fig. 3b.

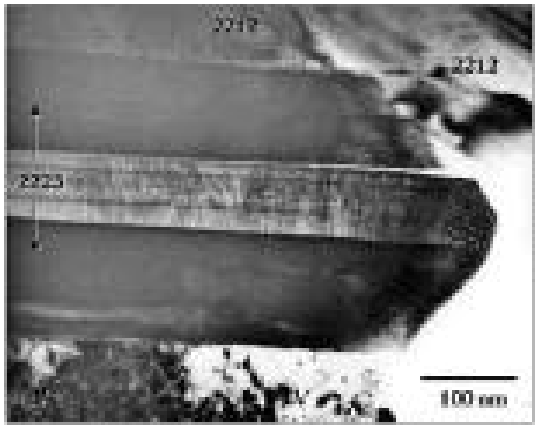

Fig. 6. TEM photomicrograph of sample subjected to $855^{\circ} \mathrm{C}$ excursion, showing large colonies of $\mathrm{Bi}-2223$ and Bi-2212 grains. 
excursion periods at a higher temperature (e.g., $855^{\circ} \mathrm{C}$ for up to $920 \mathrm{~min}$ ) resulted in substantial decomposition of the $\mathrm{Bi}-2223$ layered phase and concomitant formation of large secondary phases. Some efforts were also made to manipulate the microstructure via low- $\mathrm{pO}_{2}$ annealing (e.g., $0.02 \mathrm{~atm}$ or 0.002 atm $\mathrm{O}_{2}$ at $825^{\circ} \mathrm{C}$ ); however, this led to results similar to the extended high-temperature excursions, i.e., substantial decomposition of the Bi-2223 phase $(>80 \%)$ that could not be recovered to any significant extent by postannealing.

In summary, we find that preconverted $\mathrm{Bi}-2223$ in an $\mathrm{Ag}$ sheath back-reacts to $\mathrm{Bi}-2212$ plus secondary phases during ramp or immersion heating in $0.075 \mathrm{~atm} \mathrm{O}_{2}$. This decomposition can be arrested by use of 0.002 atm $\mathrm{O}_{2}$ during heating. Extended heat treatment at $825^{\circ} \mathrm{C}$ in 0.075 atm $\mathrm{O}_{2}$ re-forms nearly all of the $\mathrm{Bi}-2223$ phase, but does not produce any significant grain growth. Intermittent temperature dipping to $780^{\circ} \mathrm{C}$ does not visibly affect the microstructure of the layered phase. Extended excursions at elevated temperature (to $855^{\circ} \mathrm{C}$ in $\left.0.075 \mathrm{~atm} \mathrm{O}_{2}\right)$ and reduced- $\mathrm{pO}_{2}$ annealing $\left(\leq 0.02 \mathrm{~atm} \mathrm{O}_{2}\right.$ at $825^{\circ} \mathrm{C}$ ) led to $\mathrm{Bi}-2223$-phase melting/decomposition, which can be only partially reversed by annealing at $825^{\circ} \mathrm{C}$. Brief temperature excursions to $855^{\circ} \mathrm{C}(0-20 \mathrm{~min})$ followed by extended annealing at $825^{\circ} \mathrm{C}$ in 0.075 atm $\mathrm{O}_{2}$ led to layered-phase grain growth, elimination of cracks, and minimal secondary-phase growth. This general type of treatment (decomposition/re-formation) offers possibilities for improving $J_{\mathrm{c}}$ and flux pinning in Ag-clad Bi-2223 composite conductors.

\subsubsection{Synthesis of $\mathrm{Bi}-\mathrm{Based} 1212$}

Several researchers have prepared $\mathrm{Bi}, \mathrm{Pb}$-based 1212 material, nominally $(\mathrm{Bi}, \mathrm{Pb})_{1} \mathrm{Sr}_{2}(\mathrm{Ca}, \mathrm{Y})_{1} \mathrm{Cu}_{2} \mathrm{O}_{\mathrm{x}}(\mathrm{Bi}-1212)$, in which the $\mathrm{Cu}-\mathrm{O}$ planes are separated by a single $\mathrm{Bi} / \mathrm{Pb}-\mathrm{O}$ layer rather than two such layers as in $\mathrm{Bi} / \mathrm{Pb}-2212$ and $\mathrm{Bi} / \mathrm{Pb}-2223$. Because the separation between its $\mathrm{Cu}-\mathrm{O}$ layers is reduced, the in-field performance of $\mathrm{Bi}-1212$ is expected to improve relative to $\mathrm{Bi} / \mathrm{Pb}-2212$ and $\mathrm{Bi} / \mathrm{Pb}-2223$. $\mathrm{T}_{\mathrm{c}}$ for these materials has been reported in the range of 18-102 $\mathrm{K}$, but some believe that the material is nonsuperconducting and attribute the superconducting transition for these materials to small concentrations of $\mathrm{Bi} / \mathrm{Pb}-2212$ in the samples.

Because of its potential for improved in-field performance, $\mathrm{Bi}-1212$ with nominal composition $\mathrm{Bi}_{0.30} \mathrm{~Pb}_{0.50} \mathrm{Sr}_{2.1} \mathrm{Ca}_{0.15} \mathrm{~A}_{0.85} \mathrm{Cu}_{2.2} \mathrm{O}_{\mathrm{x}}(\mathrm{A}=\mathrm{Y}$ or Dy) was prepared. Powders with appropriate amounts of $\mathrm{Bi}_{2} \mathrm{O}_{3}, \mathrm{PbO}, \mathrm{SrCO}_{3}, \mathrm{CaCO}_{3}, \mathrm{Y}_{2} \mathrm{O}_{3}$ (or $\mathrm{Dy}_{2} \mathrm{O}_{3}$ ) and $\mathrm{CuO}$ were calcined at $750^{\circ} \mathrm{C}$ at reduced total pressure $\left(\approx 3\right.$ torr $\left.\mathrm{O}_{2}\right)$ to facilitate decomposition of the carbonates, then were calcined at $900^{\circ} \mathrm{C}$ at ambient pressure $\left(24 \mathrm{~h}\right.$ in $\mathrm{CO}_{2}$-free air). Pellets $(\approx 1 \mathrm{~mm}$ thick) were pressed from the powders and then sintered for $24 \mathrm{~h}$ in $\mathrm{CO}_{2}$-free air at temperatures of $960-1000^{\circ} \mathrm{C}$. After sintering, the pellets were annealed for $48 \mathrm{~h}$ at $600^{\circ} \mathrm{C}$ in either flowing $\mathrm{O}_{2}$ or $\mathrm{N}_{2}$. $\mathrm{T}_{\mathrm{c}}$ was measured by magnetization, and the microstructures were examined by SEM and TEM. Because the processing temperature was relatively high for $\mathrm{Pb}$-bearing material, the rate of $\mathrm{Pb}$ loss from the powders was measured by thermogravimetric analysis (TGA).

SEM on two samples (heated for $24 \mathrm{~h}$ at $980^{\circ} \mathrm{C}$ in air, then annealed for $48 \mathrm{~h}$ at $600^{\circ} \mathrm{C}$, one sample in $\mathrm{N}_{2}$, the other in $\mathrm{O}_{2}$ ) showed them to be very similar in their overall compositions and in the composition, morphology, and fraction of their second phases. Figure 7 shows a backscattered-electron image of the sample that was annealed in $\mathrm{O}_{2}$. It consisted of a majority matrix phase $(\approx 85 \%$ by area analysis; dark gray in Fig. 7$)$ that 


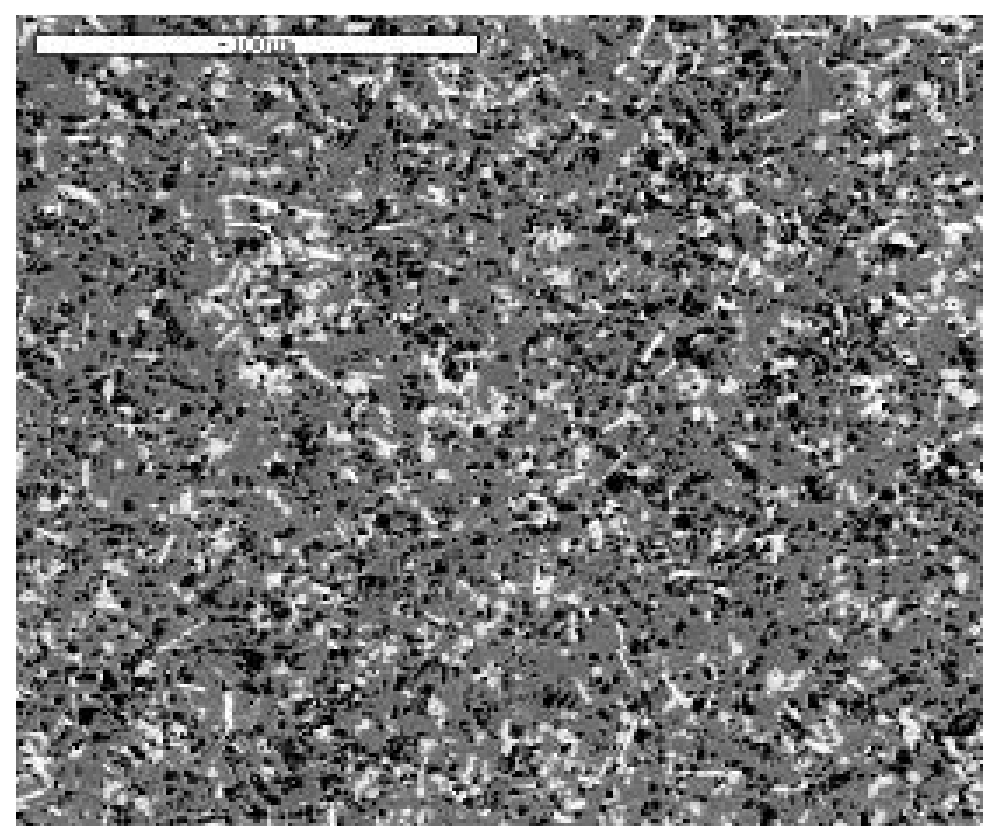

Fig. 7. Backscattered-electron image of $\mathrm{Bi}-1212$ sample heated for $24 \mathrm{~h}$ at $980^{\circ} \mathrm{C}$ in air and then annealed for $48 \mathrm{~h}$ at $600^{\circ} \mathrm{C}$ in $\mathrm{O}_{2}$.

appeared to be the desired $\mathrm{Bi} / \mathrm{Pb}-1212$ phase. In addition, there were two types of second phases that appeared bright in backscattered-electron images: roundish particles that were very rich in $\mathrm{Bi} / \mathrm{Pb}$ and $\mathrm{Sr} / \mathrm{Y}$, but contained very little $\mathrm{Ca}$ or $\mathrm{Cu}$; and needle-like particles that appeared to be $\mathrm{Bi} / \mathrm{Pb}-2212$. The area fraction for the needle-like phase was estimated to be $<10 \%$. Because the SEM was operated at $20 \mathrm{keV}$, neither the $\mathrm{Pb} / \mathrm{Bi}$ nor the $\mathrm{Sr} / \mathrm{Y}$ peaks could be resolved. In addition, the $\mathrm{Bi} / \mathrm{Pb}-2212$ needles were thin (several micrometers), so the measured composition probably reflects the composition of the surrounding matrix to some degree. It is clear, however, that the needles contain about twice as much $\mathrm{Pb}+\mathrm{Bi}$ as the matrix phase, which is consistent with the needles being $\mathrm{Bi} / \mathrm{Pb}-2212$.

The magnetization curve for $\mathrm{Bi}_{0.30} \mathrm{~Pb}_{0.50} \mathrm{Sr}_{2.1} \mathrm{Ca}_{0.15} \mathrm{Y}_{0.85} \mathrm{Cu}_{2.2} \mathrm{O}_{\mathrm{x}}$, after it was heated for $24 \mathrm{~h}$ at $960^{\circ} \mathrm{C}$ in air and annealed in $\mathrm{N}_{2}$ for $48 \mathrm{~h}$ at $600^{\circ} \mathrm{C}$, showed transitions at 22 and $85 \mathrm{~K}$. A similarly treated sample annealed in $\mathrm{O}_{2}\left(48 \mathrm{~h}\right.$ at $\left.600^{\circ} \mathrm{C}\right)$ rather than in $\mathrm{N}_{2}$, showed a single transition at $25 \mathrm{~K}$. All other samples that were prepared exhibited $\mathrm{T}_{\mathrm{C}}$ values in the range of 25-37 $\mathrm{K}$ (Table 2). The high-temperature transition $(85 \mathrm{~K})$ is typical for $\mathrm{Bi} / \mathrm{Pb}-2212$ and is consistent with photomicrographs showing small concentrations of $\mathrm{Bi} / \mathrm{Pb}-2212$. The low-temperature transitions $(22-37 \mathrm{~K})$ are near the $\mathrm{T}_{\mathrm{c}}$ of $\mathrm{Bi} / \mathrm{Pb}-2201$, which could be present if $\mathrm{Bi} / \mathrm{Pb}-2212$ is present. However, $\mathrm{Bi} / \mathrm{Pb}-2201$ was not seen in SEM or TEM examinations of the sample. The low-temperature transitions may be attributed to the majority $\mathrm{Bi} / \mathrm{Pb}-1212$ phase, but this is not clear at present. Transport measurements are being made to help resolve this issue.

TGA measurements on $\mathrm{Bi} / \mathrm{Pb}-1212$ powder showed that the rate of weight loss was nearly constant while holding for $12 \mathrm{~h}$ at $960^{\circ} \mathrm{C}$ in flowing air. Silver foil downstream from the sample collected a deposit, which XRD showed was PbO. Analysis of the deposit 
Table 2. $\mathrm{T}_{\mathrm{C}}$ of $\mathrm{Bi}-1212$ samples sintered and annealed under various conditions

\begin{tabular}{|c|c|}
\hline $\mathrm{Bi}_{0.30} \mathrm{~Pb}_{0.50} \mathrm{Sr}_{2.1} \mathrm{Ca}_{0.15} \mathrm{Y}_{0.85} \mathrm{Cu}_{2.2} \mathrm{O}_{\mathrm{x}}$ & $\mathrm{T}_{\mathrm{c}}(\mathrm{K})$ \\
\hline $24 \mathrm{~h}$ at $960^{\circ} \mathrm{C}$ (air) $/ 48 \mathrm{~h}$ at $600^{\circ} \mathrm{C}\left(\mathrm{N}_{2}\right)$ & 85,20 \\
\hline $24 \mathrm{~h}$ at $960^{\circ} \mathrm{C}$ (air) $/ 48 \mathrm{~h}$ at $600^{\circ} \mathrm{C} \mathrm{O}_{2}$ ) & 25 \\
\hline $24 \mathrm{~h}$ at $980^{\circ} \mathrm{C}$ (air) $/ 48 \mathrm{~h}$ at $600^{\circ} \mathrm{C}\left(\mathrm{N}_{2}\right)$ & 34 \\
\hline $24 \mathrm{~h}$ at $980^{\circ} \mathrm{C}$ (air) $/ 48 \mathrm{~h}$ at $600^{\circ} \mathrm{C}\left(\mathrm{O}_{2}\right)$ & 32 \\
\hline $24 \mathrm{~h}$ at $1000^{\circ} \mathrm{C}$ (air) $/ 48 \mathrm{~h}$ at $600^{\circ} \mathrm{C}\left(\mathrm{N}_{2}\right)$ & 26 \\
\hline $24 \mathrm{~h}$ at $1000^{\circ} \mathrm{C}$ (air) $/ 48 \mathrm{~h}$ at $600^{\circ} \mathrm{C}\left(\mathrm{O}_{2}\right)$ & 37 \\
\hline \multicolumn{2}{|l|}{$\mathrm{Bi}_{0.30} \mathrm{~Pb}_{0.50} \mathrm{Sr}_{2.1} \mathrm{Ca}_{0.15} \mathrm{Dy}_{0.85} \mathrm{Cu}_{2.2} \mathrm{O}_{\mathrm{x}}$} \\
\hline $24 \mathrm{~h}$ at $960^{\circ} \mathrm{C}$ (air) $/ 48 \mathrm{~h}$ at $600^{\circ} \mathrm{C}\left(\mathrm{N}_{2}\right)$ & 30 \\
\hline $24 \mathrm{~h}$ at $960^{\circ} \mathrm{C}$ (air) $/ 48 \mathrm{~h}$ at $600^{\circ} \mathrm{C}\left(\mathrm{O}_{2}\right)$ & 20 \\
\hline
\end{tabular}

showed that it contained only $\mathrm{Pb}$. The total weight loss during the isothermal hold was $\approx 3 \%$; during cooling, sample weight increased $\approx 0.5 \%$ (relative to the end of the hold at $960^{\circ} \mathrm{C}$ ). The weight gain of $0.5 \%$ was reproduced during a subsequent cycle in which the sample was heated to $960^{\circ} \mathrm{C}$ and then cooled to room temperature. The small weight gain during cooling is attributed to absorption of $\mathrm{O}_{2}$, while the weight loss at high temperature resulted from evaporation of $\mathrm{Pb}$. $\mathrm{Pb}$ loss during the isothermal hold was significant $(\approx 20 \%$ of the original lead content was lost). It is not known at this point how this affects the phase assemblage and/or doping state of the material.

In summary, large volume fractions of $\mathrm{Bi} / \mathrm{Pb}-1212$ can be readily synthesized under a wide range of conditions. These materials all exhibit superconducting transitions in the range of 22-37 K, but it remains unclear whether these transitions can be attributed to the $\mathrm{Bi} / \mathrm{Pb}-1212$ phase or an impurity phase such as $\mathrm{Bi} / \mathrm{Pb}-2212$. Transport measurements are being made to address this question. TGA measurements show that $\mathrm{Bi} / \mathrm{Pb}-1212$ loses a significant amount of $\mathrm{Pb}$ during processing. To control the loss of $\mathrm{Pb}$, which may alter the final phase assemblage and/or the doping of the material, future samples will be processed in a silver sheath.

\subsubsection{Oxygen Chemical Diffusion in Bi-2223}

The properties and microstructural development of Bi-2223 are strongly influenced by its oxygen content. As with the other high-temperature superconductors, Bi-2223 loses oxygen during heating and takes up oxygen during cooling. Although authoritative oxygen diffusion data have been published for most of the commercially important high-temperature superconductors, few data are available for $\mathrm{Bi}-2223$. This absence of 
data is due primarily to difficulty in preparing high-quality bulk specimens or sufficiently large single crystals.

Data from mechanical after-effect measurements in Bi-2223 have been ascribed to oxygen diffusion. An activation energy of $0.89 \mathrm{eV}$ was calculated from an attempt frequency of $10^{13} \mathrm{~s}^{-1}$. Oxygen tracer-diffusion studies in Bi-2212 single crystals have indicated strong anisotropy with respect to kinetics and mechanism. The diffusion coefficient within the $\mathrm{a}-\mathrm{b}$ plane could be expressed as $\mathrm{D}_{\mathrm{ab}}=8 \times 10^{-5} \exp (-1.01 \mathrm{eV} / \mathrm{kT}) \mathrm{cm}^{2} \mathrm{~s}^{-1}$; the diffusion coefficient in the $c$ direction could be expressed as $D_{c}=0.06 \exp (-2.11$ $\mathrm{eV} / \mathrm{kT}) \mathrm{cm}^{2} \mathrm{~s}^{-1}$. It was concluded that interstitial diffusion dominated within the a-b plane and that vacancy diffusion dominated in the c direction.

Dense, phase-pure, highly textured, polycrystalline specimens are ideal for many studies because properties can often be measured along the c direction and within the $a-b$ plane. Such specimens can be fabricated by sinter forging. Recently produced Bi-2223 bars have achieved high densities and excellent microstructures and properties. We report on direct measurement of the oxygen chemical diffusion coefficient (D).

Chemical diffusion was measured in pure $\mathrm{O}_{2}$ at atmospheric pressure and $700-800^{\circ} \mathrm{C}$. It was determined by measuring the weight change as a function of time, $t$, through thermogravimetric analysis. The density of the final $\mathrm{Bi}-2223$ forging was $6.105 \mathrm{~g} / \mathrm{cm}^{3}$, which is $\approx 97 \%$ of the theoretical density. The phase purity and extent of c-axis alignment were excellent (Fig. 8).

In Fig. 9, $\log \mathrm{D}$ is plotted as a function of $1000 / \mathrm{T}$. For $\mathrm{a}-\mathrm{b}$ plane diffusion, $\mathrm{D}_{\mathrm{o}}=$ $1.78 \times 10^{-3}$ and $\mathrm{E}_{\mathrm{a}}=0.96 \pm 0.17 \mathrm{eV}$. The trends of these data were consistent with those of Bi-2212: Chemical diffusion of oxygen was much faster in the $\mathrm{a}-\mathrm{b}$ plane than in the $\mathrm{c}$ direction. The activation energy in the $\mathrm{a}-\mathrm{b}$ plane was $\approx 1 \mathrm{eV}$ and the preexponential term for $\mathrm{a}-\mathrm{b}$ diffusion of oxygen was $<<1$, which is consistent with diffusion by interstitial species.

\subsubsection{Conductor Development}

Collaboration with Intermagnetics General Corporation and Southwire. For a cooperative transmission cable project, 13 spools of $\mathrm{Bi}-2223 / \mathrm{Ag}$ tape fabricated by Intermagnetics General Corporation (IGC) were received at ANL. Three of the spools (A3-1 through A3-3) were $5 \mathrm{~m}$ long and 10 spools (A10-1 through A10-10) were $10 \mathrm{~m}$ long. End-to-end, zero-field $\mathrm{I}_{\mathrm{C}}$ values at $77 \mathrm{~K}$ were $23.2-33.6 \mathrm{~A}$.

$\mathrm{J}_{\mathrm{c}}(\mathrm{H})$ measurements: Figure 10 shows representative current voltage traces at different applied fields for $\approx 4-\mathrm{cm}$-long tapes. In zero applied field, short samples A3-2 and A3-3 yielded nearly 7-8 A more than the end-to-end $I_{c}$ values measured at IGC. Sample A3-1 yielded an $\mathrm{I}_{\mathrm{c}}$ value 4 A lower than the long-length value. Measurements made in magnetic field showed an exponential decline in $I_{c}$ values from $35 \mathrm{~A}$ in zero applied field to $5 \mathrm{~A}$ in $0.2 \mathrm{~T}$ applied field for wires $\mathrm{A} 3-2$ and $\mathrm{A} 3-3$. For $\mathrm{A} 3-1$, the value decreased from 23 A to $3 \mathrm{~A}$. 


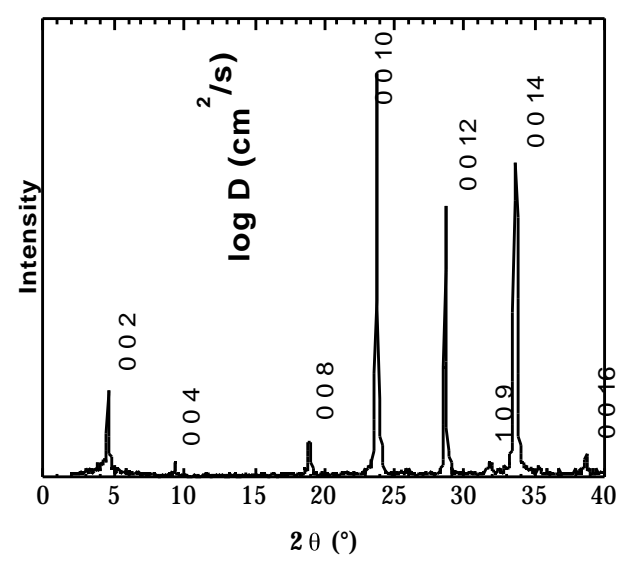

Fig. 8.

XRD spectrum for sinter-forged $\mathrm{Bi}-2223$ bar; very strong c-axis texture is evident.

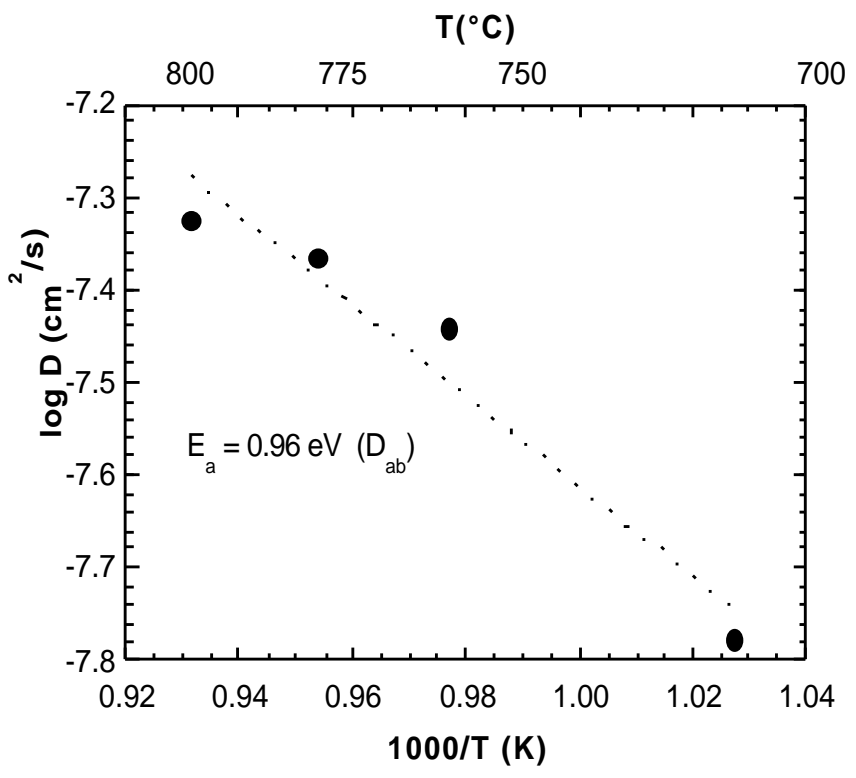

Fig. 9. Log of diffusivities of $\mathrm{Bi}-2223$ as a function of inverse temperature.

Electromechanical measurements: Short samples $\approx 6 \mathrm{~cm}$ long were cut from each spool and used for mechanical testing. Figure 11 shows the experimental arrangement for mechanical testing.

Because of the large number of plots generated from each spool of tape, those for tape A365M11A will be shown and be considered to be representative of the other tapes. Several samples from each spool were tested under tensile load until fracture occurred. Figure 12 shows a typical load-vs.-displacement curve.

To correlate the effects of mechanical deformation on transport properties, tapes were subjected to various loads $(8.9,16.0$, and $20.9 \mathrm{~N})$. Figure 13 shows transport current measurements under applied field for tape A3-3 with each increment of loading. Significant degradation of $\mathrm{I}_{\mathrm{c}}$ began to occur between 16.0 and $20.9 \mathrm{~N}$ of loading. 


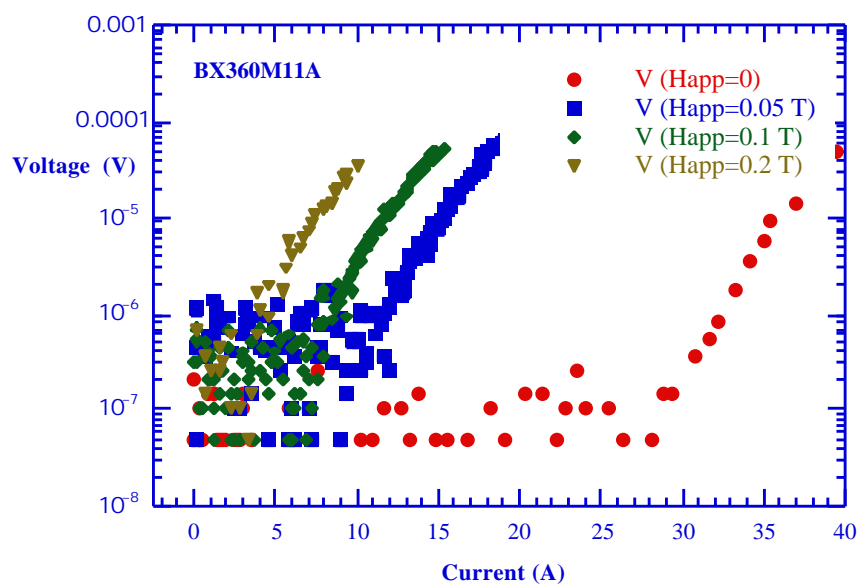

Fig. 10. Current voltage traces for Tape A3-2.

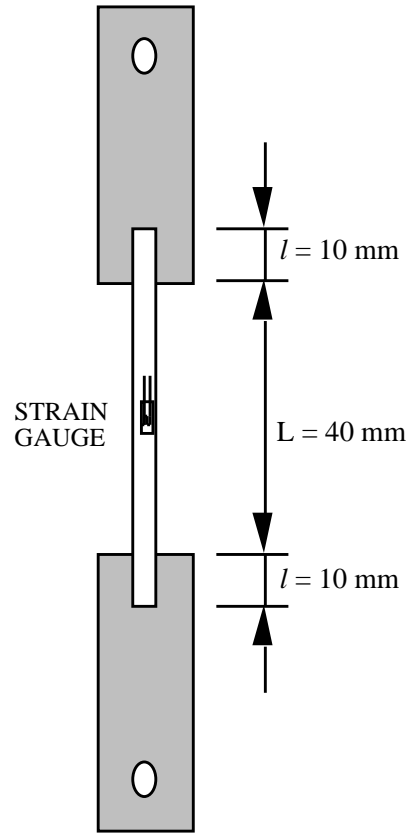

Fig. 11. Schematic arrangement of $\mathrm{Bi}-2223$ tape used for tensile testing.

Strain gauges were mounted on several short samples from each spool in order to obtain stress-vs.-strain curves. Figure 14 shows the curve obtained for tape A3-1 tape. The level of strain at different loads is indicated on the curve. It can be seen that an 8.9-N tensile load causes $\approx 0.1 \%$ strain and an $16.0-\mathrm{N}$ tensile load causes $\approx 0.2 \%$ strain. Figure 15 shows the load-vs.-strain and $\mathrm{I}_{\mathrm{c}}-\mathrm{vs}$.--strain data. 


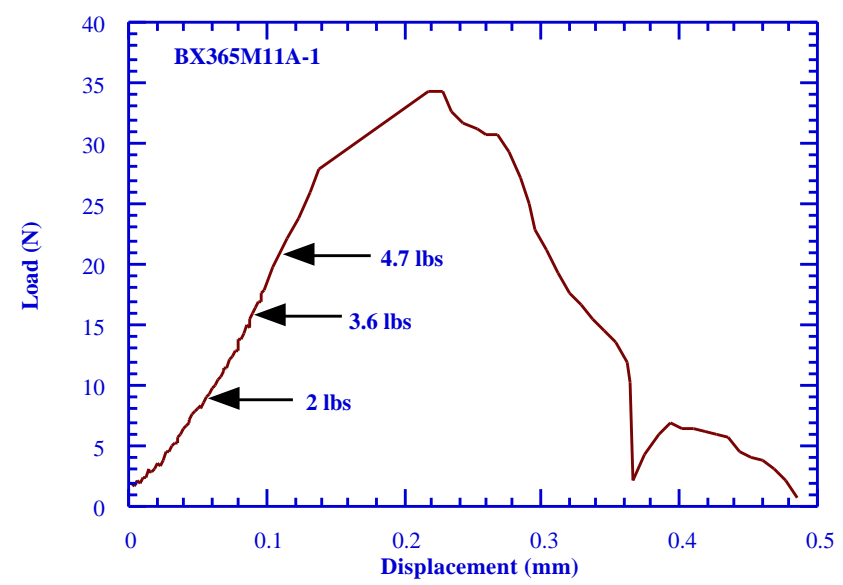

Fig. 12. Load vs. displacement for Bi-2223 Tape A3-3.

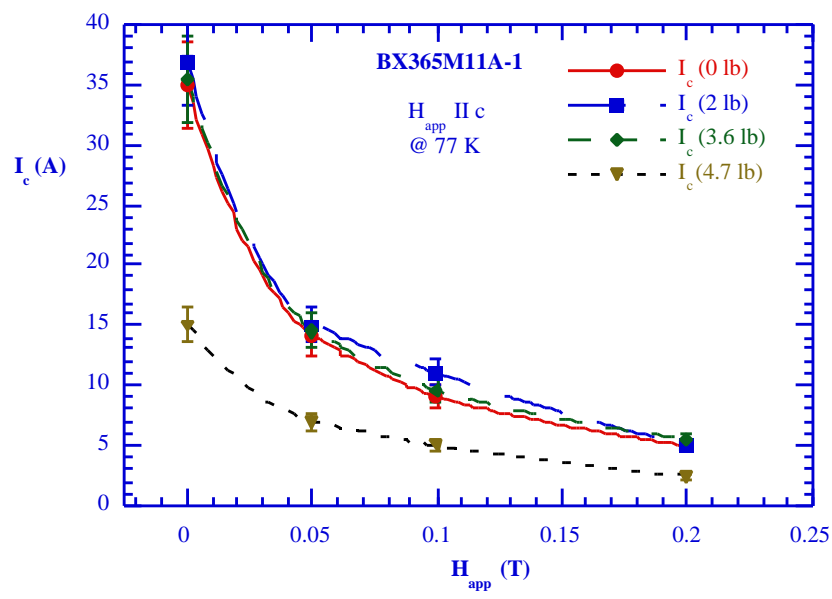

Fig. 13. Transport critical current vs. applied magnetic field for Tape A3-3 at no load, 8.9, 16.0, and $20.9 \mathrm{~N}$ loads.

Longer lengths of tapes were used to test transport current properties after being wound on a G-10 mandrel of 3.8- $\mathrm{cm}$ diameter at $30^{\circ}$ angle. A3 tapes were measured at 1-pitch length and A10 tapes were measured at 2-pitch length. Measurements were done at the first half, the second half, and over the entire length. Figure 16 shows representative $I_{c}$ data for A3 tapes over 1-pitch length.

In summary, mechanical testing indicated that tapes can withstand up to $16 \mathrm{~N}$ of tensile loading without detriment. Transport current measurements on tapes after 8.9, 16.0 , and $20.9 \mathrm{~N}$ loading showed that $20.9 \mathrm{~N}$ loading reduces $\mathrm{I}_{\mathrm{c}}$ value from $35 \mathrm{~A}$ to $15 \mathrm{~A}$ in zero field. 


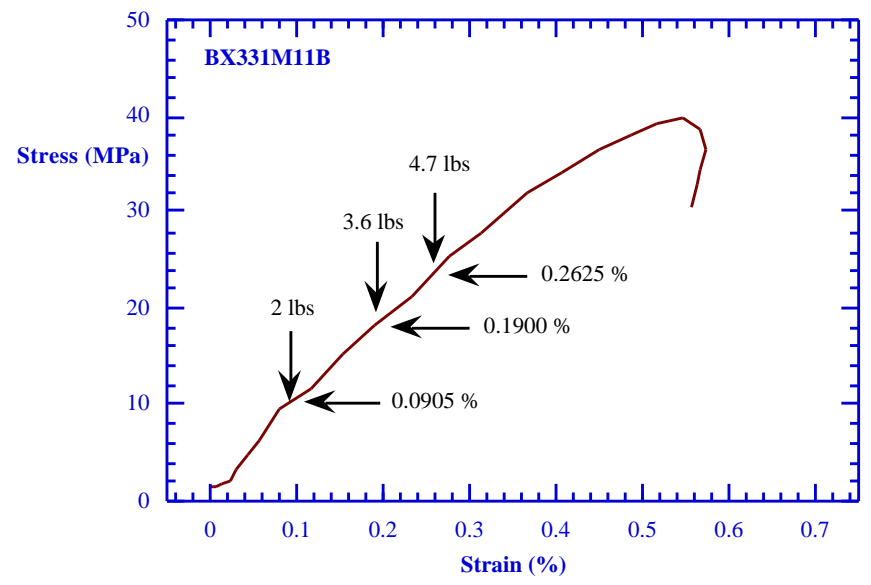

Fig. 14. Stress vs. strain for Tape A3-1.

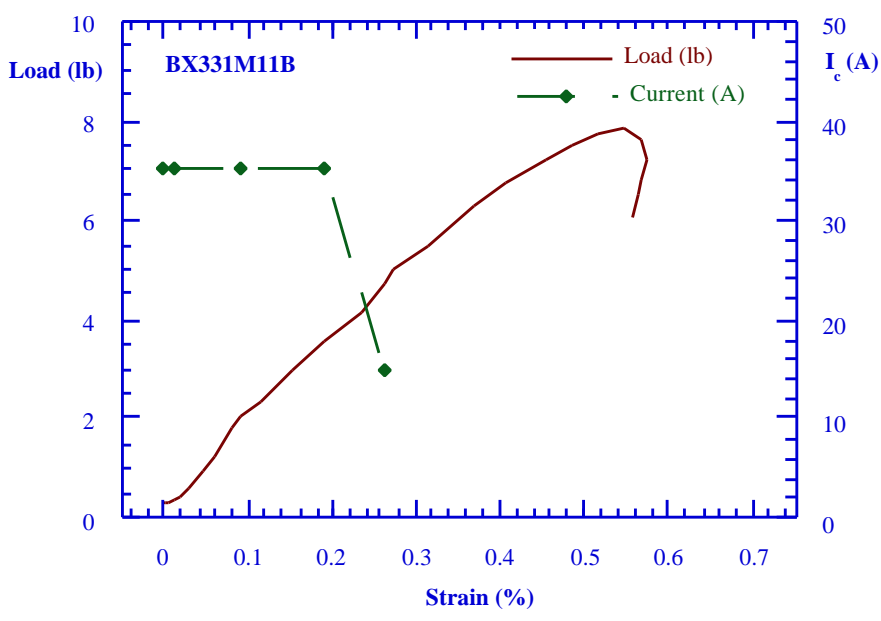

Fig. 15. Load and critical current as function of strain.

Stress-strain measurements indicated that a $16-\mathrm{N}$ load causes $\approx 0.2 \%$ strain and a $20.9-\mathrm{N}$ load causes $0.26 \%$ strain. Significant reduction in transport $\mathrm{I}_{\mathrm{c}}$ began to occur at $\approx 0.2 \%$ deformation.

AC Losses in Bi-2223 Tapes. Much of this work was conducted in collaboration with the All-Russian Electrical Engineering Institute in Moscow. We present results of study of a 5-m-long AC transmission line with $1 \mathrm{kA}$ current capability at $77 \mathrm{~K}$. Primary attention was paid to the current characteristics and AC losses in individual tapes and in the core of the cable. The losses were measured as a function of $\mathrm{AC}$ magnetic field amplitude in 


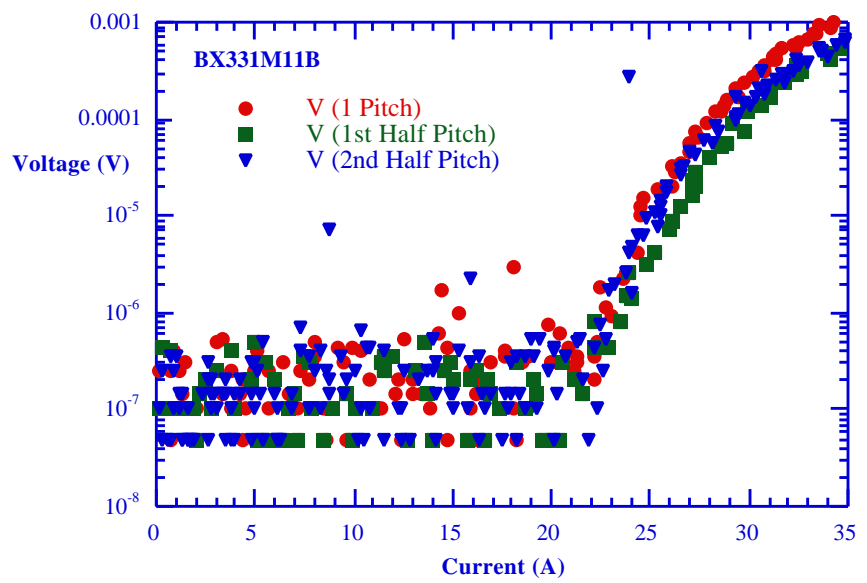

Fig. 16. Current vs. voltage for Tape A3-1 wound on 3.8-cm-diameter mandrel.

various orientations of magnetic field with respect to the plane of the tape and filaments. Hysteresis losses were close to the losses in the AC regime, which indicates that eddy-current losses in tapes may be neglected when compared with hysteresis losses. We designed and constructed from multifilamentary Bi-2223 tapes a 5-m-long AC transmission line. The core contained 120 tapes for the forward line and 120 tapes for the backward line.

The Bi-2223/Ag tapes were fabricated by IGC. The tapes were $4.4 \mathrm{~mm}$ wide and $0.19 \mathrm{~mm}$ thick and contained 37 filaments. Transport $\mathrm{J}_{\mathrm{c}}$ of an elementary tape was measured by a four-point-probe method. V-I characteristics of several tapes, each $\approx 50 \mathrm{~cm}$ long, were measured with a nanovoltmeter (Keithley-181). To obtain a field configuration close to that of the transmission line, we prepared the sample as two strips (like direct and back-line wires) with a thin insulating layer between them. On the other end, two current leads were soldered. To check the homogeneity of the current capability, we mounted four voltage contacts. The distance between contacts was $36.5 \mathrm{~cm}$ for each pair. An increase in voltage with an increase in current at $\mathrm{I} \approx 15$ and $20 \mathrm{~A}$ for a pair of strips was observed. At higher currents, the voltage increased sharply as a power function of I. The exponents were $\approx 25$ and 15 for the two strips. At I $>30$ A, the exponents decreased. With a $1 \mu \mathrm{V} / \mathrm{cm}$ criterion, the critical currents were 26 and $22 \mathrm{~A}$.

Measurement of losses by magnetization methods: Samples for DC magnetization measurements were cut from a Bi-2223/Ag tape that contained 37 filaments. Each sample consisted of 10 pieces of tape, each measuring $0.5 \times 0.44 \times 0.019 \mathrm{~cm}$. The pieces were joined with tape, so that they were insulated from each other, and the resulting sample shape was approximately a cube. A vibrating-sample magnetometer was used to measure the magnetization loops $\mathrm{M}(\mathrm{H})$. Measurements were performed at $77 \mathrm{~K}$ in magnetic fields to $1 \mathrm{kOe}$. Three DC magnetic field orientations were considered: (a) field parallel to the tape surface but perpendicular to the filaments, (b) field directed along the filaments, and (c) field perpendicular to the plane of the tape. 
Magnetization loops for the three orientations show that the areas defined by field orientations $\mathrm{a}$ and $\mathrm{b}$ are similar to each other, whereas the area defined by $\mathrm{c}$ is much greater. This difference may be related to the orientation of our experiment, in particular, to the demagnetization factors caused by the flat form of the filaments. Hysteresis losses were determined from the area of the magnetization loops for orientation a in a DC regime. The dependence of hysteresis losses $\mathrm{P}_{\mathrm{H}}$ on the maximum $\mathrm{H}$ per cycle is presented in Fig. 17. This dependence is typical for hard superconductors. At low fields of $\mathrm{H}<50$ Oe, losses are proportional to $\mathrm{H}^{3}$ (dashed line in the figure). At higher fields, the exponent tends to be unity.

To define the total losses in the tape, we measured the dependence of the imaginary part $\chi$ " of the AC susceptibility as a function of the magnitude $\mathrm{h}_{\mathrm{o}}$ of the AC magnetic field for two directions of $h$ with respect to the filaments. The losses $\mathrm{Q}$ (in watts) are related to $\chi$ " by the simple equation $\mathrm{Q}=10^{-7} \chi^{\prime \prime} \mathrm{h}_{\mathrm{o}}^{2} \mathrm{Vf} / 4$, where $\mathrm{V}$ is the sample volume in $\mathrm{cm}^{3}$. Dependence on AC field amplitude of the total losses per $1 \mathrm{~m}$ of tape length for two directions of applied magnetic field is shown in Fig. 18. Curve 1 in the figure corresponds to $\mathrm{h}$ parallel to the filaments, and Curve 2 was obtained for $\mathrm{h}$ perpendicular to the filaments.

Measurement of losses by AC current methods: To model conditions similar to those of a transmission line, we studied the AC transport properties of the double-strip sample. The V-I characteristics of the sample were measured for the AC current: $I=I_{O} \cos (\omega t)$, where $\omega / 2 \pi=\mathrm{f}$, at frequencies $\mathrm{f}$ of 13 and $60 \mathrm{~Hz}$. A lock-in amplifier PAR 124A was used in differential mode to detect the voltage that was in phase with the current and shifted on $\pi / 2$ with respect to it. The reference was a signal taken from a noninductive resistor in series with the superconducting tape. A drop in AC voltage is defined by the transport resistivity (at high currents), remagnetization losses, and eddy current losses in a normal metal. The losses were calculated by using the in-phase voltage drop $\mathrm{V}$ as follows: $\mathrm{Q}=\mathrm{V} \times \mathrm{I}$ (Fig. 19).

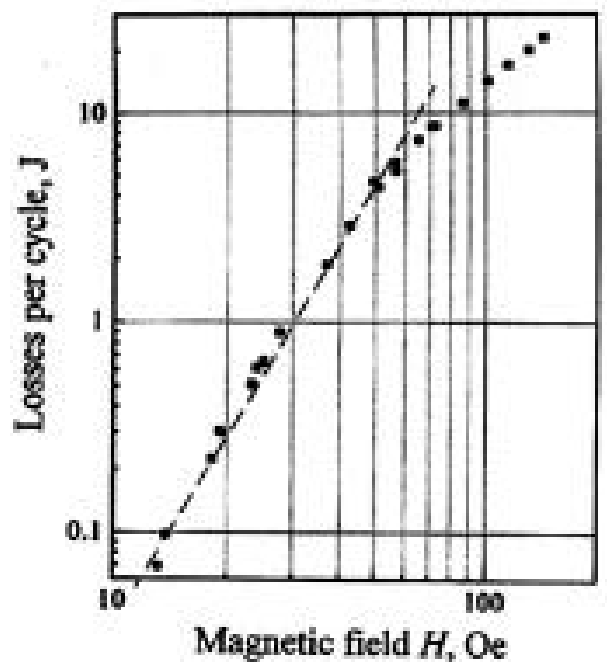

Fig. 17.

Hysteresis losses per cycle per cubic meter as a function of magnetic field $\mathrm{H}$. Dashed line corresponds to cubic relationship. 


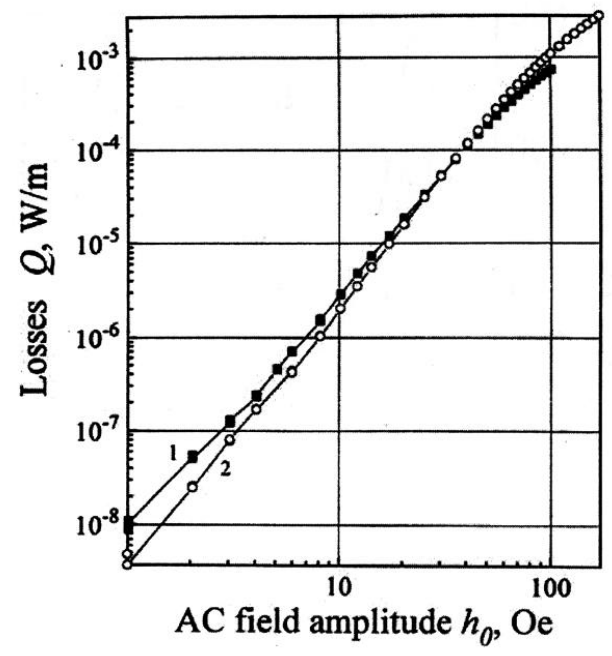

Fig. 18. AC losses for orientations where AC field amplitude $h_{0}$ is parallel to filaments (Curve 1 ) and perpendicular to them (Curve 2).

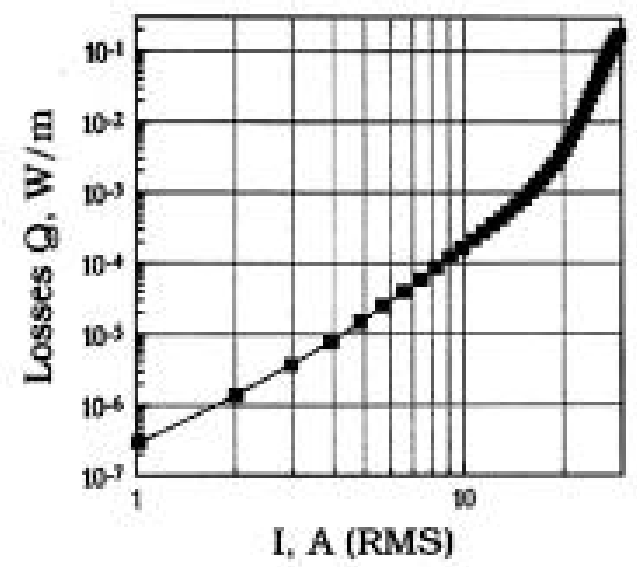

Fig. 19. AC losses of tape at $60 \mathrm{~Hz}$ as a function of RMS AC current.

We have considered the data for losses obtained by three methods. All of the data are in good agreement over the entire field range presented. The field range limit corresponds to $\approx 1 / 2$ of the critical current value, i.e., the typical operating range of the transmission line current. The results from DC and AC magnetization methods correlate well. Differences are more pronounced at high currents. Indeed, if some portions of the measured tape contained bad clusters, the losses should be higher in these regions of the tape than in other homogeneous regions and thus lead to an increase in losses, which was observed in our experiment. 
Testing the model conductor and defining its characteristics: We divided the material into forward and backward lines according to material volume. The lines contained 120 5-m-long tapes. The current capability of each strand of the cable was almost the same $(\approx 160 \mathrm{~A})$. We used 50-mm-wide Mylar tape as the base of each strand. Each tape in the strand was inserted into 120-mm-wide slits cut across the Mylar tape. The strands exhibited good rigidity, and each superconducting tape in the strand was well spaced and electrically isolated from the other tapes.

Wire transposition in the current core does not significantly alter the performance of transmission cables. Each pair of strands was put on a thin Mylar tape in an X-form, wound on a stainless steel former. Such construction enabled the transposition of the tapes in two strands, but the adjacent three pairs of strands were not transposed. The outer diameter of the current core after winding was $\approx 48 \mathrm{~mm}$. In accordance with the superconducting current capability, we expected to obtain a total line critical current of $\approx 160 \times 6=960$ A.

To test the transmission line, we put the current core into a cryogenic envelope. We used the short-circuit testing regime by connecting all of the flexible copper wires from one end of the current core. The current and potential wires from the other end were inserted through a cap on the cryogenic envelope into the testing circuits. All flexible current wires were electrically isolated.

We used a transformer current input to supply current to the core. To measure AC losses in the transmission line, the potential probes were connected to the input of an amplifier. The measurements were performed at $77 \mathrm{~K}$.

In the first stage of testing, we determined the DC V-I characteristics of individual strands. DC current was supplied to neighboring strands in series, and the voltage drop on each strand was measured by a Keithley 181 nanovoltmeter. Figure 20 shows the V-I characteristics of all strands. Despite matching the equal current capability of strands, dispersion is noticeable. Contact resistivity changes from one strand to another over the range of 110 to $120 \mathrm{~mW}$.

We tested the current core of the transmission line in the $\mathrm{AC}$ regime at $60 \mathrm{~Hz}$. The control voltage, which fed the primary winding of the transformer, was approximately sinusoidal. Inductance of the current core with the copper current leads was $\approx 0.3 \mathrm{mH}$. The voltage drop on the copper current leads (the component of the control voltage that was in phase with the current) was linear with the current. To obtain the active voltage that was related to the superconducting current core, we subtracted the linear part of the voltage drop. The dependence $\mathrm{V}(\mathrm{I})$ is close to a power function with the exponent $\mathrm{n}=3.12$. Figure 21 shows the dependence of $\mathrm{AC}$ losses $\mathrm{W}$ in the current core as a function of the transport current I. The experimental results are indicated by closed circles. The losses W increased with I proportional to $\mathrm{I}^{\mathrm{n}}$, with $\mathrm{n}=3.8$ at currents $\mathrm{I}<700 \mathrm{~A}$. At higher currents, losses increased faster. Such behavior is related to the transition to the resistive state.

The AC transmission line model carried an AC transport current of up to $1000 \mathrm{~A}$ at $77 \mathrm{~K}$. An increase in current from zero to $1100 \mathrm{~A}$ did not lead to serious disturbances in loading characteristics of the superconducting current core. 


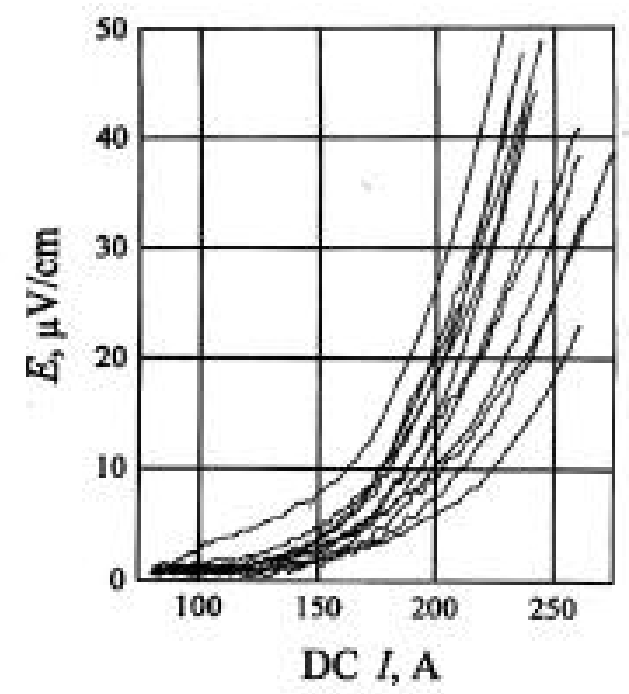

Fig. 20. V-I characteristics of tapes.

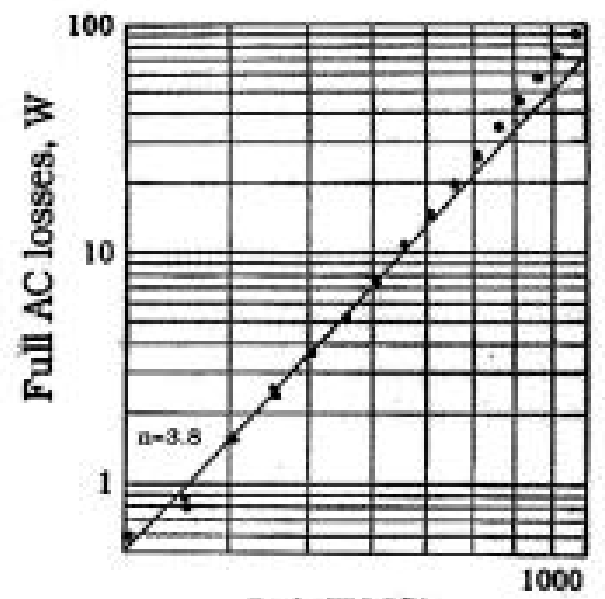

Fig. 21.

Full AC losses in line model (points) and fit function that corresponds to data in a low-current region (solid line).

\section{A (RMS)}

Shielded $\mathrm{Bi}-2223$ Superconducting Tapes. We fabricated a new composite tape in which the primary function of the central Ag-sheathed $\mathrm{Bi}-2223$ filaments was to conduct transport current. A Y-123 thin film was deposited on the top of the Ag-sheathed Bi-2223 tape to shield the applied magnetic field and protect the Bi-2223 filaments. A 100-nm-thick layer of $\mathrm{SrTiO}_{3}$ (STO) was deposited as a buffer. The critical current densities of the Y-123-coated, Ag-sheathed Bi-2223 tapes were higher than those of an uncoated tape. However, the $\mathrm{Y}-123$ thin film exhibited $\mathrm{a} \mathrm{T}_{\mathrm{c}} \approx 72 \mathrm{~K}$ and a broad transition region that shifted the effect to lower temperatures. Furthermore, pole figures showed widely spread $a-b$ planes along the rolling direction, indicating high-angle grain boundaries that diminished the magnitude of the effect. Microstructural observations revealed platelike grains of $\mathrm{Y}-123$ with fine growth ledges in the thin film that was heat treated, in contrast with the microstructure of an as-coated thin film that showed large twinned grains. From the processing point of view, the results showed that heat treating 
Y-123 thin film according to the Bi-2223 tape schedule was compatible with and beneficial for Y-123. These preliminary results may provide a basis for further improvements in processing long-length $\mathrm{Bi}-2223$ tapes for high-field applications.

Figure 22 shows the composite conductor. It is possible that an oxygen deficiency in the as-coated state lowered the $\mathrm{T}_{\mathrm{c}}$. Figure 23 shows $\mathrm{X}$-ray diffraction (XRD) scans taken from the top surfaces of as-coated and heat-treated tapes. In the as-coated state, only peaks for Y-123 and Ag are present. However, the presence of another peak in the heat-treated tape is characteristic of $\mathrm{BaY}_{2} \mathrm{O}_{4}$ phase. These results were confirmed by EDS analysis.

Figure 24 shows the $\mathrm{J}_{\mathrm{c}}$ dependence of a heat-treated $\mathrm{Bi}-2223$ tape coated with a Y-123 thin film on applied magnetic field $\left(\mathrm{H}_{\mathrm{app}}\right)$ and $\mathrm{T}$; the reference tape in the figure was uncoated but heat treated under identical conditions. The magnetic field was applied parallel to the c-axis. $J_{\mathrm{c}}$ was determined from the width of the hysteresis loop. The increase in $J_{\mathrm{C}}$ is attributed to the magnetic contribution of the $\mathrm{Y}-123$ thin film to the $\mathrm{Bi}-2223$ grains. The increase is more pronounced at lower temperatures and at higher fields. In our case, the effect is shifted to lower temperatures because of the critical temperature of Y-123 thin film. Also, the magnitude of the effect is affected by the misorientation of the Y-123 grains. However, the results of our work show that this approach can be used to enhance $\mathrm{J}_{\mathrm{c}}$ of $\mathrm{Bi}-2223$ at higher temperatures and in higher magnetic fields. Moreover, from the processing point of view, the results show that heat treating Y-123 thin films according to the Bi-2223 tape schedule is compatible with $\mathrm{Y}-123$. We believe that the effect will be more pronounced when substrates with better textures, such as Ag alloys, are used.

Internal Oxidation of Ag-alloy Sheaths. Increasing the strength and stiffness of Ag-alloy sheaths of high-temperature superconductor wires and tapes has been identified as a critical technological need. Strengthening is accomplished by internally oxidizing the solute species of the alloy. To examine the fundamental kinetics of the internal-oxidation

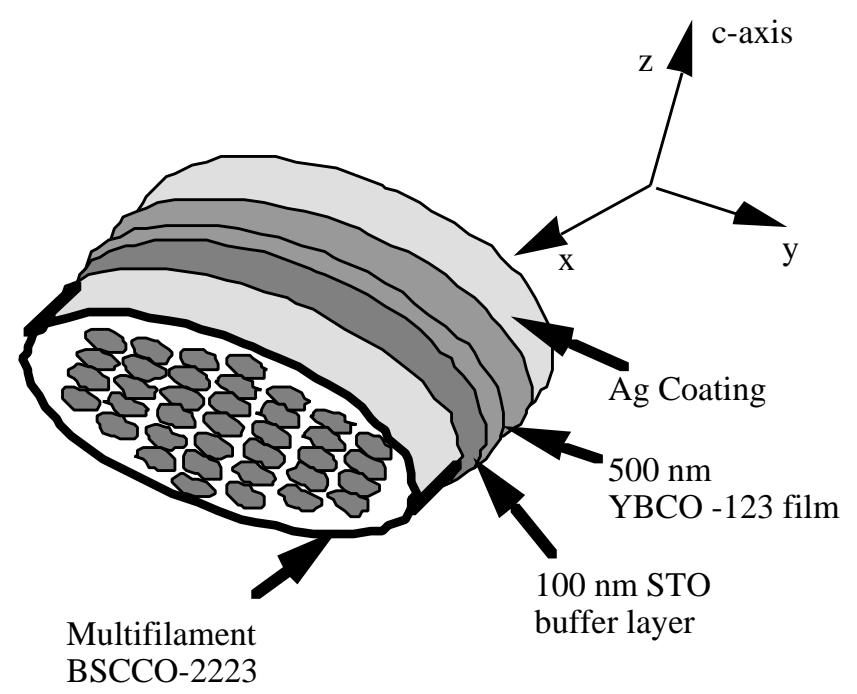

Fig. 22. Schematic diagram of $\mathrm{Y}$-123-shielded Bi-2223 multifilament tape. 


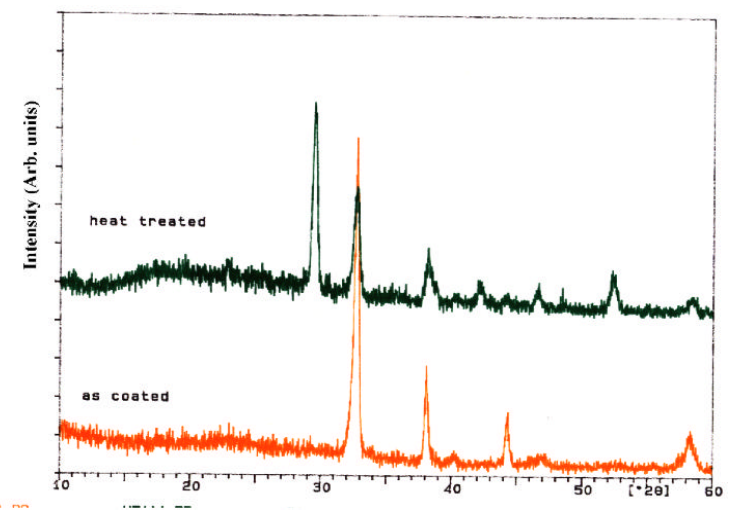

Fig. 23. XRD scans taken from top surfaces of as-coated and heat-treated tapes, showing a peak in heat-treated tape characteristic of $\mathrm{BaY}_{2} \mathrm{O}_{4}$.

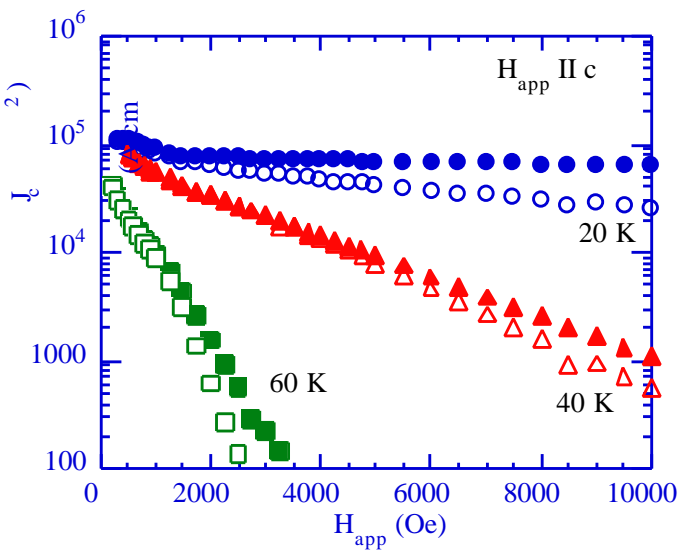

Fig. 24.

$\mathrm{Jc}$ at 20,40 , and $60 \mathrm{~K}$ as a function of magnetic field applied parallel to c-axis: solid triangles represent $\mathrm{Bi}-2223$ tape coated with Y-123 thin film and heat treated; open squares represent uncoated and heat-treated reference tape.

process, we are working with Prof. Michael McNallan of the University of Illinois at Chicago. In this study, square coupon samples (thickness $=0.5 \mathrm{~mm}$ and mass $\leq 0.5 \mathrm{~g}$ ) of Ag, $\mathrm{Ag} / 1.2$ at.\% $\mathrm{Mg},(\mathrm{AgMg})$ and $\mathrm{Ag} / 0.25$ at.\% Mg-0.25 at.\% (AgMgNi) Ni were prepared from cold-rolled and polished sheets. TGA was performed at $450-825^{\circ} \mathrm{C}$, and hardness profiles were determined by Knoop indentations. Microstructures were examined by optical microscopy and SEM.

The internal oxidation of both alloys exhibited nearly identical activation energies (0.810 eV for $\mathrm{Ag}-\mathrm{Mg}$ and $0.828 \mathrm{eV}$ for $\mathrm{Ag}-\mathrm{Mg}-\mathrm{Ni}$ ) and rate constants (Fig. 25). The maximum oxygen contents of both alloys were found to be superstoichiometric (i.e., $\mathrm{R}=\mathrm{O} / \mathrm{Mg}>1.0$ ). The maximum values at lower temperatures (e.g., $\mathrm{R}=1.25$ at $500^{\circ} \mathrm{C}$ ) were higher than those at higher temperatures (e.g., $\mathrm{R}=1.05$ at $800^{\circ} \mathrm{C}$ ). We define this phenomena as a cluster size effect: Smaller cluster sizes have higher $\mathrm{R}$ values and are attributed at lower temperatures from magnesium's inability to diffuse to common areas; larger clusters have lower $\mathrm{R}$ values and more closely resemble the MgO structure. 


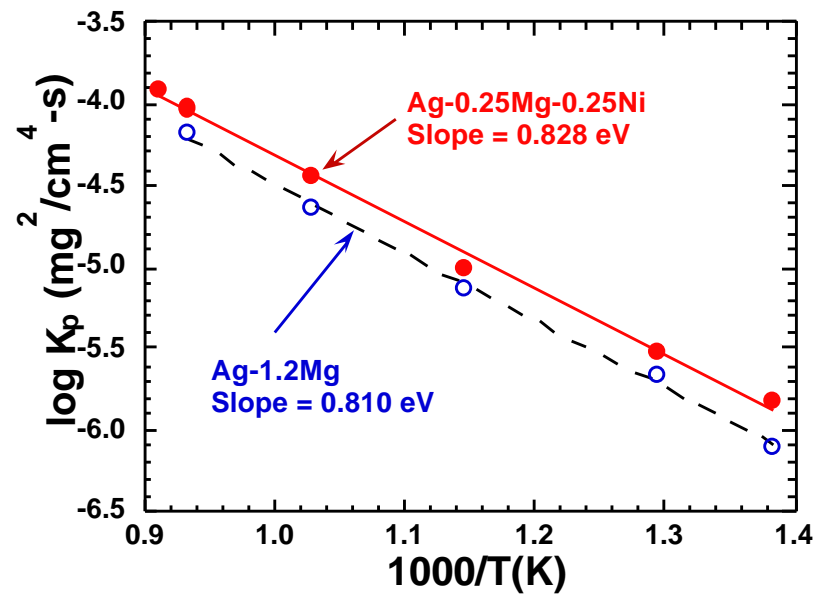

Fig. 25. Activation energy for internal oxidation of Ag alloys.

A comparison of oxygen diffusion in pure Ag with the kinetics of internal oxidation of the Ag alloys is shown in Fig. 26. Oxygen diffusion in Ag was tentatively found to be $\approx 60$ times faster at $825^{\circ} \mathrm{C}$ and nearly 400 times higher at $500^{\circ} \mathrm{C}$. More analysis is required to compare diffusion and internal oxidation directly.

Microhardness and strength profiles of the AgMg alloy were found to change considerably, depending on the treatment temperature and time. Lower processing temperatures $\left(450-500^{\circ} \mathrm{C}\right)$ yielded significantly smaller grain sizes and more uniform microstructures as compared to higher temperatures, where smaller grains were found near the surface and larger ones in the interior. Knoop hardness profiles for AgMg, 99.9\% $\mathrm{Ag}$, and $99.999 \% \mathrm{Ag}$ treated at $825^{\circ} \mathrm{C}$ in $8 \% \mathrm{O}_{2}$ for $17 \mathrm{~h}$ are given in Table 3 . The AgMgNi alloy yielded fairly uniform microhardness profiles and microstructures in this temperature regime, with slightly larger grain sizes at higher temperatures. In both alloys, $\mathrm{Mg}$ was found to migrate to the grain boundaries at low temperatures, $450-500^{\circ} \mathrm{C}$, where it formed an $\mathrm{Ag}_{3} \mathrm{Mg}$ intermetallic phase. At higher temperatures, SEM observations did not reveal the presence of $\mathrm{Mg}$ in the grain boundaries. It is believed to exist as very small $\mathrm{MgO}$ precipitates at the grain boundaries or as $\mathrm{Mg}-\mathrm{O}$ clusters within the $\mathrm{Ag}$ matrix. In the AgMgNi, Ni was found to precipitate in the form of well-dispersed small spherical particles that increased in size with annealing time and temperature.

\section{$2.2 \mathrm{Y}-\mathrm{Ba}-\mathrm{Cu}-\mathrm{O}$ Superconductors}

\subsubsection{Coated-Conductor Development}

Ion-beam-assisted deposition (IBAD) is a promising method for fabricating long lengths of coated conductors. It enables deposition of highly oriented template layers on randomly oriented metallic substrates. These layers are also buffers that act as diffusion barriers between the metal substrate and $\mathrm{YBa}_{2} \mathrm{Cu}_{3} \mathrm{O}_{\mathrm{x}}(\mathrm{Y}-123)$ conductor film. We have used IBAD techniques to deposit highly textured buffer layers of yttria-stabilized $\mathrm{ZrO}_{2}$ (YSZ) on polycrystalline metals using an assisting off-normal low-energy ion beam incident on the substrate during deposition. We have also deposited $\mathrm{CeO}_{2}$ (ceria) buffers on YSZ. 


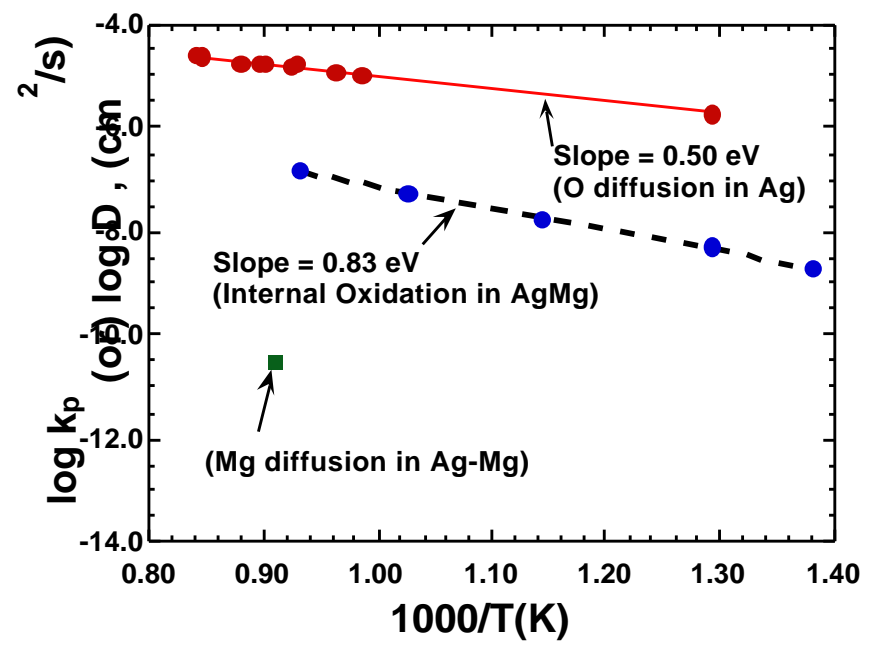

Fig. 26. Comparison of internal oxidation and $\mathrm{Mg}$ diffusion in AgMg and oxygen diffusion in Ag.

Table 3. Room-temperature microhardness values vs. cross-sectional position for internally oxidized $\mathrm{Ag}-1.12$ at.\% $\mathrm{Mg}$ and pure $\mathrm{Ag}$, in $8 \% \mathrm{O}_{2}$ at $825^{\circ} \mathrm{C}$ for $17 \mathrm{~h}$

\begin{tabular}{cccc}
\hline & \multicolumn{3}{c}{ Average Knoop hardness $\left(\mathrm{kg} / \mathrm{mm}^{2}\right)$} \\
\cline { 2 - 4 } Position from & & & \\
surface (mm) & $\mathrm{Ag} / 1.12 \% \mathrm{Mg}$ & $99.9 \% \mathrm{Ag}$ & $99.999 \% \mathrm{Ag}$ \\
\hline $0-60$ & 534.0 & 42.2 & 53.9 \\
(near surface) & 551.0 & - & 56.5 \\
$60-120$ & 155.0 & - & - \\
\multirow{2}{*}{$120-180$} & 141.0 & - & - \\
& 127.0 & 35.7 & 45.0 \\
250 & 117.0 & - & 57.7 \\
& 91.6 & - & - \\
\hline
\end{tabular}

YSZ Layers. YSZ films were deposited by electron beam evaporation. During deposition, the substrates were bombarded by an assisting 300-eV ion beam of $\mathrm{Ar} / 10 \%$ oxygen ions at varying angles by using an $8-\mathrm{cm}$ Kaufman-type DC ion source with molybdenum grids. The ion beam was neutralized with a tungsten filament at $110 \%$ of the ion beam current to ensure complete neutralization. A calibrated quartz-crystal monitor $(\mathrm{BCM})$ was used to determine the gross deposition rate. A schematic diagram of the deposition configuration is shown in Fig. 27. 


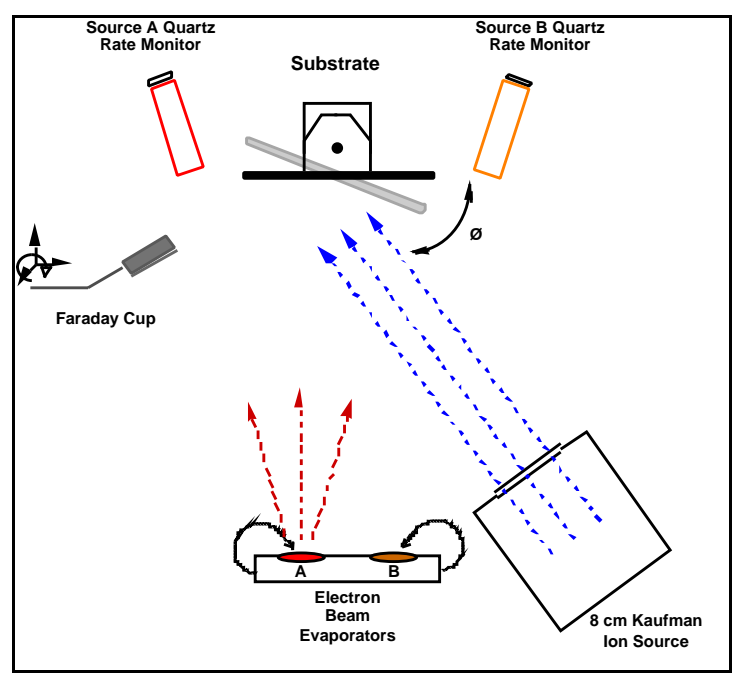

Fig. 27. Schematic representation of IBAD electron beam evaporator chamber.

The ion flux was measured with a Faraday cup at normal incidence to the ion beam. An electron-screening grid on the Faraday cup was negatively biased to $20 \mathrm{~V}$ to repel the neutralizer electrons and thus accurately measure the ion flux. IBAD YSZ films were deposited under ion bombardment at angles of $29-48^{\circ}$ with respect to the substrate parallel to a gross thickness of $\approx 1 \mu \mathrm{m}$. As-received and polished Hastelloy $\mathrm{C}$ and as-received 304 stainless steel sheets were used as substrates.

The base pressure of the IBAD chamber before evaporation and ion bombardment was $10^{-7}$ torr, which rises to an operating pressure of $9 \times 10^{-5}$ torr during deposition. All depositions were initiated at room temperature; during IBAD, the substrate temperature rose from 25 to $\approx 200^{\circ} \mathrm{C}$. For most films, a constant ion-to-atom flux ratio ( $r$ value) of 2.8 was maintained for various bombardment angles.

Biaxial texture was characterized with a four-circle X-ray diffractometer. Out-of-plane texture was determined by the full-width-at-half-maximum (FWHM) of $\Omega$ scans of the YSZ (200) reflection, and in-plane texture was measured by the FWHM of $\phi$ scans of the YSZ (111) reflection.

All films were (200) biaxially oriented with the (111) pole reflection in the direction of the ion beam. The out-of-plane FWHM showed no clear angular dependence; values ranged from 5 to $10^{\circ}$. The out-of-plane texture of the polished substrates was $\approx 2^{\circ}$ better than that of the unpolished substrates. No clear difference was noted between as-received substrates. Figure 28 shows a typical $\Omega$ scan for an IBAD YSZ film deposited on stainless steel.

The in-plane texture of IBAD YSZ in this study showed a reproducible dependence on the angle of ion bombardment. Minimization of the in-plane FWHM $\phi$ occurred at $35^{\circ}$, which corresponds to the (111) channeling angle for cubic YSZ, thus lending support to the selective resputtering theory. The in-plane texture of IBAD YSZ on as-received SS substrates equaled that of the polished Hastelloy substrates. Figure 29 shows a $\phi$ scan for an IBAD YSZ film deposited on polished Hastelloy at $35^{\circ}$, with a FWHM of $17^{\circ}$. This is an 


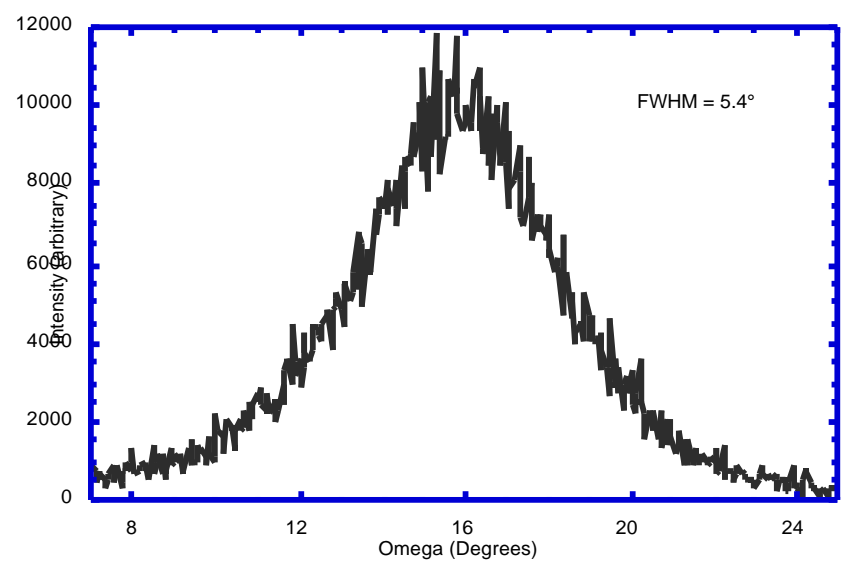

Fig. 28. (200) $\Omega$ scan for IBAD YSZ film on as-received stainless steel.

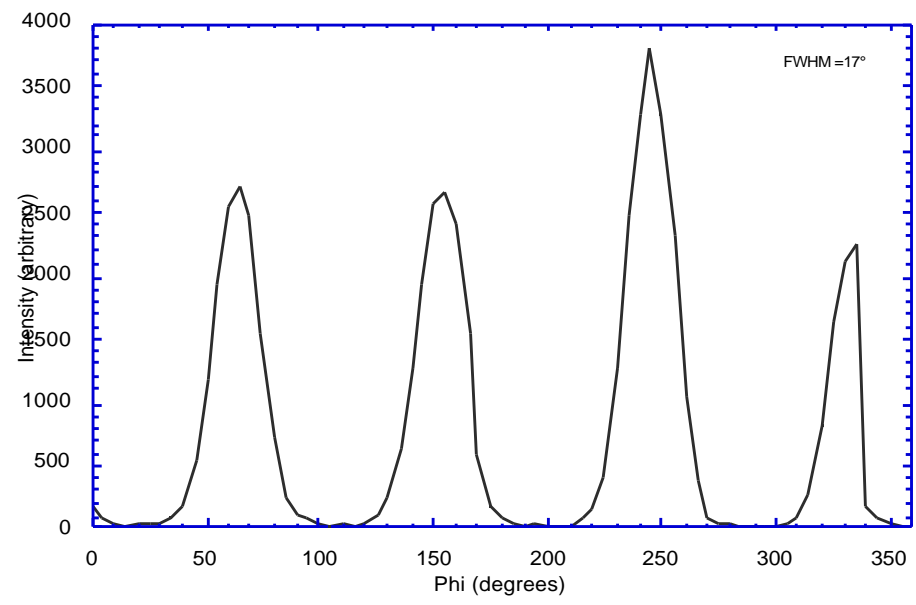

Fig. 29. X-ray $\phi$ scan for IBAD YSZ film on polished Hastelloy at $35^{\circ}$.

important result because scale-up production of IBAD YSZ tapes for coated conductor applications would benefit from fewer processing steps. The reason for this effect is not completely understood, and more studies are in progress to determine a possible mechanism.

In-plane texture was studied for ion/atom flux ratios ( $r$ value) between 0 and 3.8 by varying the ion flux and the atomic flux for a bombardment angle of $35^{\circ}$. YSZ films deposited without ion assistance were largely amorphous, with broad low-intensity (111) and (200) peaks. IBAD with a $300-\mathrm{eV}$ ion beam and flux ratios between 1 and 0.5 produced mixed orientations, dominated by (111) grains. The ions presumably provided enough surface mobility to the adatoms of YSZ to preferentially grow with a $<111>$ normal to the surface. Films with a (200) biaxial orientation were observed when the $r$ value exceeded 1 .

It has been shown that the degree of orientation of thin films under IBAD increases asymptotically to a critical value of $r\left(r_{c}\right)$, at which more material is sputtered than deposited. It is, therefore, not surprising that the degree of in-plane texture should also 
increase to a critical value of $r$. Figure 30 shows a series of (111) pole figures for IBAD YSZ films deposited when the $r$ values were $1.3,2.8$, and 3.5. In all cases, the (111) pole is oriented in the direction of the bombarding beam.

The in-plane texture of IBAD YSZ films improves linearly with film thickness in the range between 0.3 and $1 \mu \mathrm{m}$ gross film thickness. This effect was observed in IBAD YSZ films when $r$ values were 1.3 and 2.8 and confirms that the texture improves with film thickness, presumably as grains oriented with the ion beam overgrow the misoriented grains.

In summary, the texture of YSZ films deposited by the IBAD technique was investigated and found to depend on ion-bombardment angle, film thickness, ion-to-atom flux ratio, and substrate material. An ion-bombardment angle of $35^{\circ}$ with respect to the substrate parallel produced a minimum in-plane texture, which was as low as $17^{\circ}$ in a $1-\mu \mathrm{m}$-thick film. Higher ion/atomic flux ratios improved the in-plane texture. Additional work is needed to find a value of $r_{c}$ at which texture no longer improves due to complete resputtering. This will require an optimized IBAD system capable of ion flux values greater than $350 \mu \mathrm{A} / \mathrm{cm}^{2}$ at the substrate.

Ceria Layers. $\mathrm{CeO}_{2}$ layers have proved to be beneficial to IBAD-fabricated coated conductors. A reduction in the lattice mismatch between the IBAD YSZ buffer layer and the overlying Y-123 in coated conductors (4\% lattice mismatch) can be obtained through

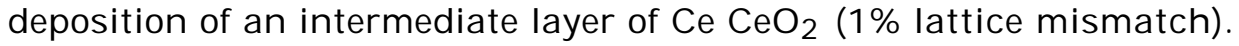

$\mathrm{CeO}_{2}(99.5 \%$ pure) and $\mathrm{Ce}(99.9 \%$ pure) were used as source materials in a high-vacuum dual-electron-beam evaporator with a base pressure of $1 \times 10^{-7}$ torr. YSZ substrates, $0.8 \mu \mathrm{m}$ thick, grown on polished Hastelloy $\mathrm{C}$ by IBAD were used as substrates. All $\mathrm{CeO}_{2}$ films were grown to a thickness of $\approx 500 \AA$ at a deposition rate of $30 \AA / \mathrm{min}$, as measured by a $\mathrm{QCM}$. The oxygen partial pressure was measured by a residual gas analyzer and controlled with a calibrated mass-flow controller.

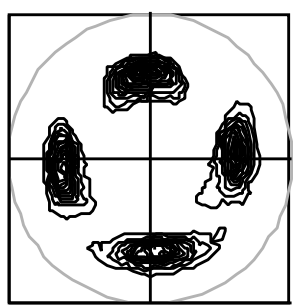

(a)

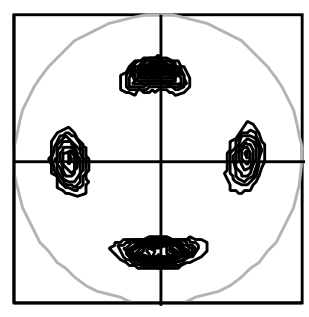

(b)

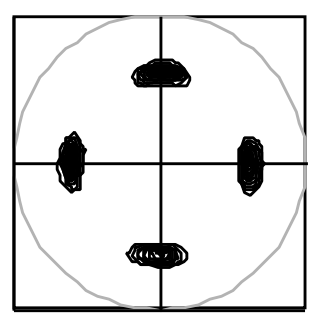

(c)

Fig. 30. (111) X-ray pole figures for IBAD YSZ films deposited to gross thickness of $1 \mu \mathrm{m}$ with $r$ values of (a) 1.3 , (b) 2.5 , and (c) 3.8 . 
Epitaxial growth of $\mathrm{CeO}_{2}$ films, from the oxide source, on (200) IBAD YSZ tapes required a substrate temperature $>600^{\circ} \mathrm{C}$ with a $\mathrm{pO}_{2}>10^{-5}$ torr. $\mathrm{CeO}_{2}$ from the metallic Ce source were epitaxially grown on IBAD YSZ tapes as low as $100^{\circ} \mathrm{C}$, without the addition of oxygen. A residual $\mathrm{pO}_{2}$ of $2 \times 10^{-8}$ torr was sufficient to oxidize the Ce to cubic $\mathrm{CeOx}$ $(\mathrm{x}>1.72)$. Figure 31 shows an XRD spectrum for $\mathrm{CeO}_{2}(200) / \operatorname{IBAD} \mathrm{YSZ}(200)$.

The crystallinity of $\mathrm{CeO}_{2}$ films grown on IBAD YSZ tapes improved with an increase in the substrate temperature (Figs. 32 and 33). The upward trend at higher substrate temperatures in Fig. 33 of IBAD YSZ intensity with respect to the overlying $\mathrm{CeO}_{2}$ is due either to interlayer diffusion or an increase in intensity for the underlying IBAD YSZ tape as it anneals at the elevated temperatures.

In summary, $\mathrm{CeO}_{2}$ cap layers were grown epitaxially on IBAD YSZ tapes at substrate temperatures as low as $100^{\circ} \mathrm{C}$, without the addition of oxygen by use of reactive evaporation of metallic Ce. Epitaxial films from the oxide source required higher substrate temperatures and oxygen partial pressures.

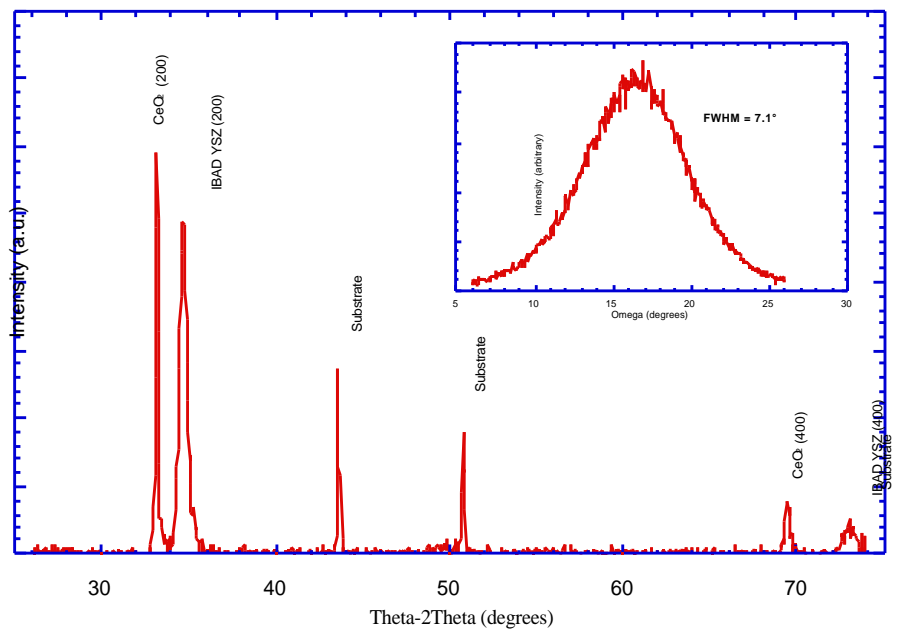

Fig. 31. $\theta-2 \theta$ XRD spectrum for $\mathrm{CeO}_{2}$ grown on an IBAD YSZ tape at $300^{\circ} \mathrm{C}$, with inset $(200)$ rocking curve.

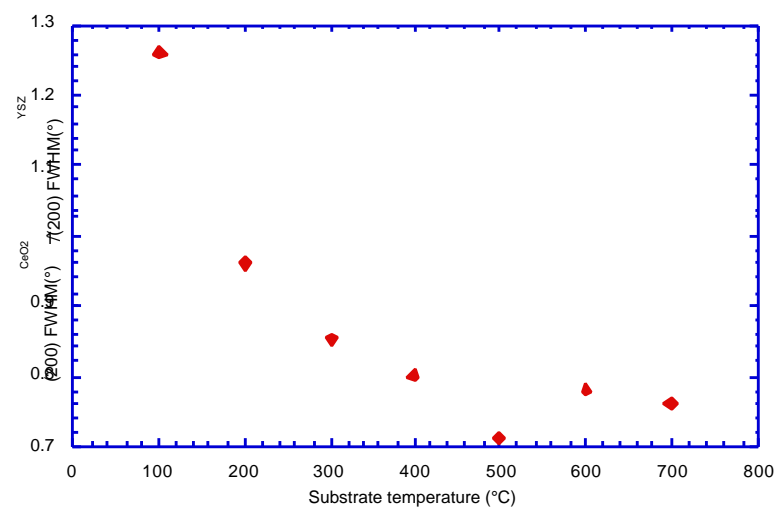

Fig. 32. FWHM of (200) rocking curve of $\mathrm{CeO}_{2}$ normalized to FWHM (200) of YSZ substrate. 


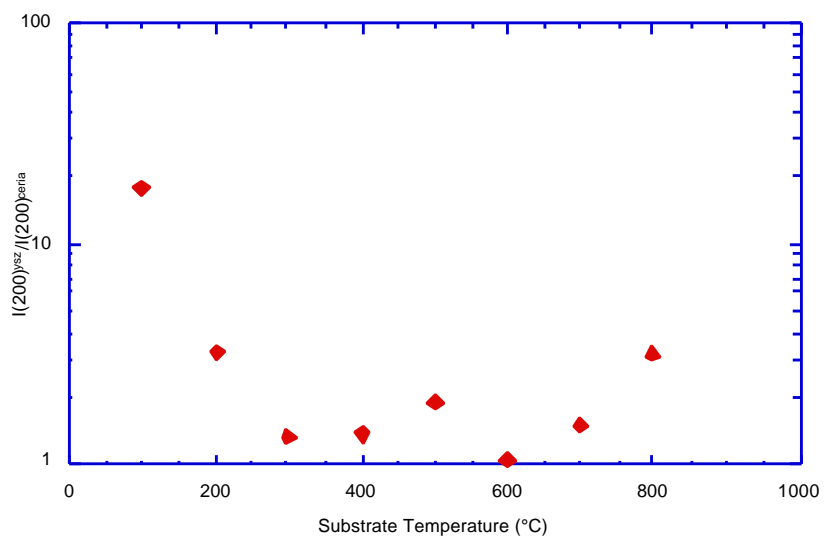

Fig. 33. Intensity ratio of YSZ (200)/ $\mathrm{CeO}_{2}(200)$ peaks vs. substrate temperature.

\subsubsection{Noise Properties of $\mathrm{Y}-123$ Films}

While the critical current, $I_{c}$, and current-voltage characteristics, I(V), give us useful information on flux-creep dissipation, measurements of noise voltage can reveal more subtle features of the vortex dynamics. These can provide clues to the important properties and characteristics associated with flux-creep dissipation. The ultimate goal is to connect them to the microstructure and thus propose fabrication schemes to improve $\mathrm{I}_{\mathrm{C}}$.

Our recent analysis of the vortex-motion noise in high-temperature superconductors showed that the moving vortices consist of vortex line segments rather than well-connected vortex lines throughout the sample thickness. The vortex lines can break up into segments near the transition temperature $\mathrm{T}_{\mathrm{c}}$ because the coupling energy between $\mathrm{CuO}$ bilayers is comparable to the thermal energy. This emphasizes the importance of strong Josephson inter-bilayer coupling to enhance $\mathrm{I}_{\mathrm{c}}$. One might expect that pinning defects that span the entire sample thickness could alter this picture. The present studies use columnar defects in order to illuminate the effect of correlated pinning defects on the breakup of vortex line segments. Noise was measured in epitaxial Y-123 films as a function of temperature, magnetic field, and bias current. Results were compared before and after introducing columnar defects by ion irradiation. Vortex motion always exhibits a noise peak, which occurs at a field-dependent temperature. With use of a simple model to analyze the noise, the average size of the moving vortex bundle is proportional to the ratio of the noise power to the average rate of vortex flow, the time-averaged (DC) voltage, $V_{d c}$. We found an increased noise peak magnitude after irradiation and interpreted it as an increased bundle size. This could be due to an increased length of vortex segments due to the interaction with, e.g., pinned vortices in columnar defects.

Magnetic field and current dependencies of the noise can provide additional insights into the effects of columnar defects. After irradiation, the noise peak was almost independent of magnetic field, B, when it is less than about half of the matching field (i.e., equivalent density of columnar pinning defects), $\mathrm{B}_{\mathrm{f}}$, but dropped by a factor of 3 for $\mathrm{B} \approx 2 \mathrm{~B}_{\mathrm{f}}$. However, for $\mathrm{B}<0.5 \mathrm{~B}_{\mathrm{f}}$, the bundle size is significantly enhanced in the irradiated sample. This enhancement disappears at higher currents or fields and is absent in the unirradiated sample (Fig. 34). 


\subsubsection{Grain-Boundary Transport and Microstructure}

Grain Boundaries. Improvements in the performance and economics of coated conductors must consider the dissipation mechanism in grain boundaries. The flat, bulk grain boundaries that we made provide a basis for understanding the role of the meandering grain boundaries found in deposited films and the intrinsic properties unrelated to the meander. We are now patterning the low-angle 001 tilt boundaries found in coated conductors. An example of what can be learned is found in our recent results on the temperature and field dependence of the critical current in $90^{\circ}$-tilt bulk grain boundaries. Although this geometry of these grain boundaries is rather specific, we observed an interesting behavior (described below) that may be a general property of grain boundaries.

The inset of Fig. 35 shows the geometry used with the magnetic field parallel to the grain-boundary plane, as well as to the $\mathrm{CuO}$ bilayers of the grains. The field dependence of $\mathrm{I}_{\mathrm{C}}$ at $84 \mathrm{~K}$ is shown in Fig. 35; for the lower curve, the applied field was turned on after cooling in zero field (ZFC), while for the upper curve the sample was cooled from above $T_{C}$ in the field applied (FC). Below $1 \mathrm{~T}, \mathrm{I}_{\mathrm{C}}$ for $\mathrm{FC}$ is much higher than that of ZFC and the ZFC $I_{C}$ shows a peak near $0.1 \mathrm{~T}$. This hysteretic behavior of $I_{C}$ can be understood in terms of Josephson-like vortices in the grain boundary being pinned by an array of pinned Abrikosov vortices in the grains. According to this model, the higher $\mathrm{I}_{\mathrm{C}}$ for $\mathrm{FC}$ would be the result of a higher Abrikosov vortex density (essentially the applied field) in the banks of the grain boundary. For $\mathrm{ZFC}$, such a complete saturation of Abrikosov vortices in the banks would occur only in high fields, due, e.g., to surface barriers and bulk pinning. Further work is needed to fully justify this preliminary conclusion.

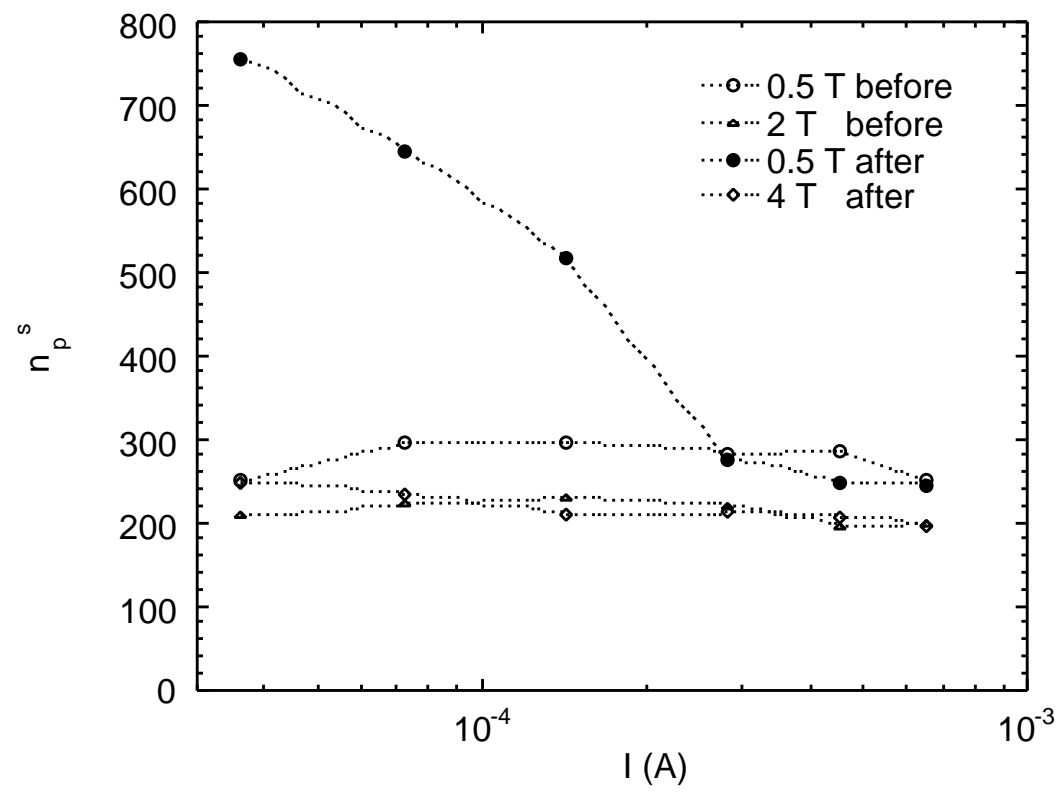

Fig. 34. Noise peak in $\mathrm{Y}-123$ films vs. current for conditions shown in legend. 


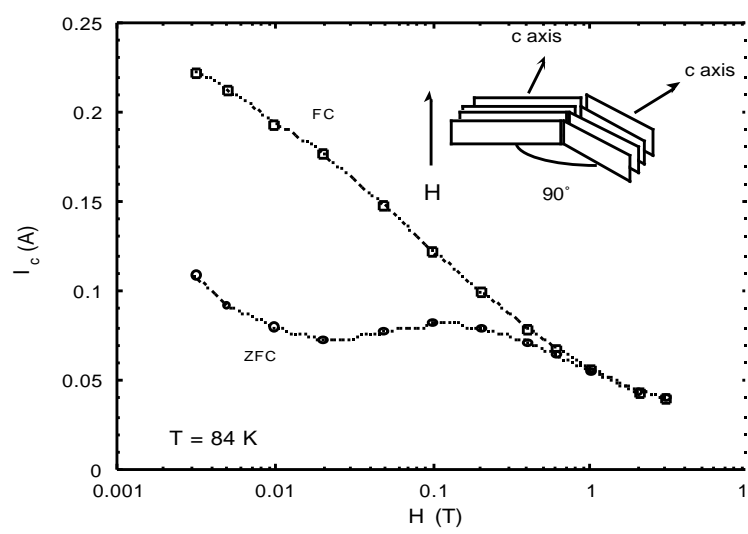

Fig. 35.

Critical current vs. applied magnetic field for grain boundary shown in inset.

Bicrystal Grain Boundaries in Y-123. Grain-boundary studies in Y-123 have focused mainly on [001] tilt misorientations, because these are the easiest nongeneral GB misorientations to fabricate. Real-world conductor applications, however, require GBs with several other types of misorientations; these can range from [001] twist to [100] tilt to general misorientations that cannot be simply described about a low-index rotation axis or pure twist or tilt grain-boundary plane. It is of keen interest to determine if certain types of misorientation axes or grain-boundary planes are more favorable to current transport than others. This information can be used to better focus the key alignment parameters for conductor fabrication. In this report, we describe a fabrication technique that allows us to explore the role of the rotation axis on superconducting properties. Specifically, we create samples that contain not only a [001] tilt grain boundary of some preselected misorientation angle $\theta$, but a [100] tilt grain boundary of that same $\theta$, and an additional [100] tilt grain boundary of $90^{\circ}-\theta$. Because of symmetry, the maximum misorientation that has been generally discussed for [001] tilt type GBs is $45^{\circ}$, because the samples are twinned and $\mathrm{Y}-123$ is approximated as tetragonal $(\mathrm{a} \approx \mathrm{b})$.

It has been demonstrated previously that cubic Nd-123 (actual composition at surface: $\mathrm{Nd}_{1.25} \mathrm{Ba}_{1.75} \mathrm{Cu}_{2.6} \mathrm{Al}_{0.4} \mathrm{O}_{\mathrm{x}}$ ) seeds can be used to create five-domain $\mathrm{Y}-123$ samples, wherein the five domains are misoriented $90^{\circ}$ from each other. In an ideal five-domain sample, as in Fig. 36, the center domain (in this paper labeled "A") grows with the c-axis parallel to the vertical direction of the cylindrical pellet, while the other four domains grow with the c-axis parallel to the radius of the pellet (labeled " 1 " or " 2 ", and the other two domains misoriented $180^{\circ}$ are degenerate). By combining this concept with dual-seeding concepts of creating [001] tilt bicrystals, we can form a variety of grain boundaries. From Fig. 37a, we see that with dual cubic seeds and perfectly straight grain boundaries, the only [100] tilt GBs that can be formed are of angle $\theta$ that is equal to the misorientation of domains $\mathrm{A}$ and $\mathrm{A}^{\prime}$ (dictated by the placement of the seeds). However, if the GB macroscopically meanders (Fig. 37b), we may find that regions intersect, forming 90 $-\theta$ [100] tilt grain boundaries. As the center c-axis normal domains (A and $\mathrm{A}^{\prime}$ ) grow in a pyramidal shape, they will eventually touch at some distance vertically from the top of the pellet, forming the [001] tilt GBs, like those formed by flat Nd-123 seeds. 


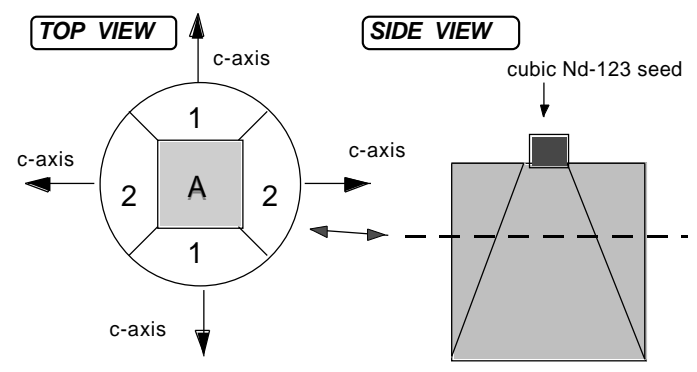

Fig. 36.

A single cubic $\mathrm{Nd}-123$ seed generates five domains in the Y-123 pellet, with three symmetrically unique orientations misoriented $90^{\circ}$ from each other.

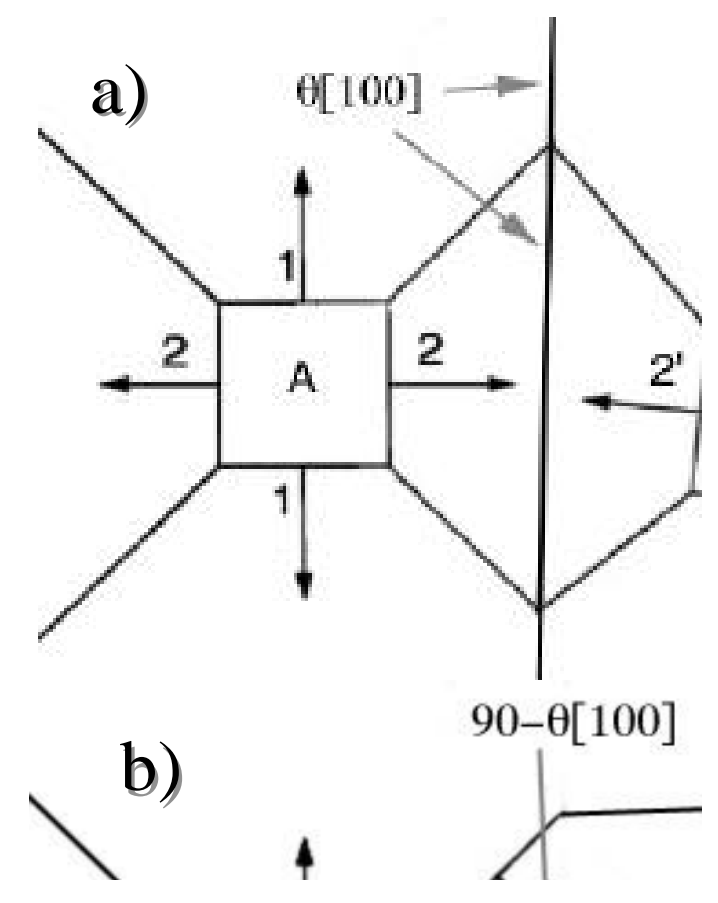

Fig. 37. Regions $A$ and $B$ represent c-axis-normal regions of two five-domain samples; numbered regions all have c-axis in the plane of the paper in the direction of their arrows. When $A$ and $B$ regions are misoriented by $\theta$ and with straight grain boundaries, as in (a), they form $\theta[100]$ GBs at intersections of regions $1-1^{\prime}$ or $2-2^{\prime}$. If the grain boundaries macroscopically meander so that they separate regions 1-2' or 2-1', as in (b), a $90^{\circ}-\theta[100]$ grain boundary is formed. When regions $A$ and $B$ grow together vertically lower in the pellet, a $\theta[001]$ tilt grain boundary is formed.

Several pellets of composition $75 \% \mathrm{Y}-123$ and $25 \% \mathrm{Y}_{2} \mathrm{BaCuO}_{5}$ (211), with a small amount of Pt, were prepared with $\mathrm{Nd}-123$ seeds. The angle $\theta$ was measured with of a light microscope (Fig. 38a). Photographs were taken of the sample surface to determine the post-melt-texture misorientation of the seeds (Fig. 38b). Samples were then cut with a diamond saw. To verify the misorientation angles, the samples were etched in a $1 \%$ solution of $\mathrm{HBr}$ in ethanol for $\approx 45 \mathrm{~s}$ in preparation for obtaining electron backscatter patterns (EBSPs). Software analysis was used to index the EBSPs based on the known crystal structure to obtain the misorientation between the two regions. 

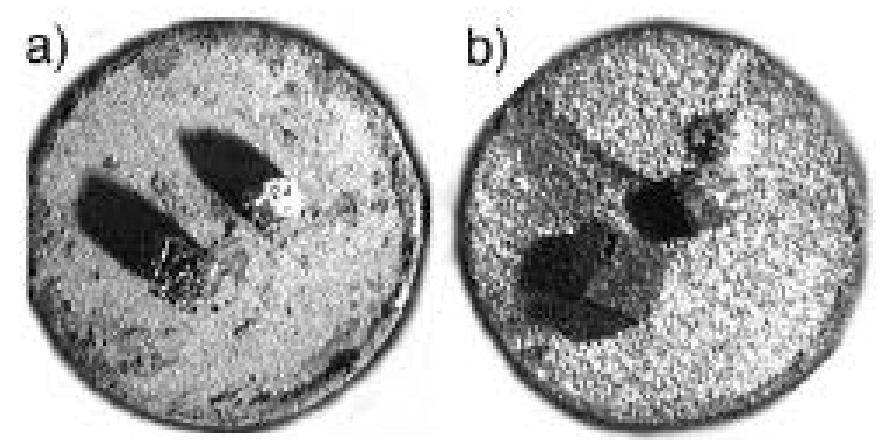

Fig. 38. (a) Two cubic seeds are placed at a misorientation angle $\theta$ on the surface of a $\mathrm{Y}-123$ pellet before melt-texturing. (b) After meltexturing, the angle between the seeds is re-recorded.

Figure 39 shows the Kikuchi patterns from representative areas in grains A and A'. Because the difference in patterns can be quite subtle, high-quality patterns were required in order to obtain a positive identification of the orientation. Subtle differences in the patterns, such as the distance between parallel bands and the number of bands intersecting at a location, help to define the sample orientation. Patterns were taken as close to the $\theta[100]$ and $90^{\circ}-\theta[100]$ GBs as possible. Table 4 summarizes the range of experimental data; an approximated value for the angle and axis pair was obtained by taking a median value for the rotation angle and noting the low index axis that the data had in common. The $\theta[001], \theta[100]$, and $90^{\circ}-\theta[100]$ values are roughly obtained as predicted. A slightly lower misorientation angle for the $90^{\circ}-\theta$ might be expected, but besides an experimental error of $\approx 1^{\circ}$, the slight mosaic spread in the melt-textured material can change the value by a few degrees from that predicted.

The GB misorientation angles have matched the predicted values to within a few degrees, which is reasonable considering the mosaic nature of melt-textured Y-123. We have found that imperfect cubic Nd-123 seeds and growth development actually can help extend the range of boundaries that can be grown. Slight mosaic spreads of a few degrees are beneficial as well, because small and essentially homogeneous regions can be isolated for transport via laser cutting, but the larger boundary region as a whole allows a small spread of misorientations to be investigated within one sample. These samples and others have been used to study effects of grain-boundary structure on superconducting properties.

The $I_{c}$ and $J_{c}$ of a grain boundary are macroscopic quantities that reflect not only the intrinsic coupling across the boundary (which would determine electron conduction) but also external parameters, such as flux motion along the boundary (which contributes to measured dissipation). Both factors are important in determining the transport properties of a grain boundary.

An illustration of the difficulty in isolating the key factors that result in reduced critical currents across grain boundaries in high-temperature superconductors is shown in Fig. 40. In this figure, previously reported results of $\mathrm{J}_{\mathrm{c}}$ measured across single grain boundaries in thin film bicrystals and in bulk bicrystals are plotted as a function of the misorientation angle $\theta$ between the grains. Qualitatively, the data for the thin film and bulk bicrystal grain boundaries are similar. However, significant differences are noted in the 


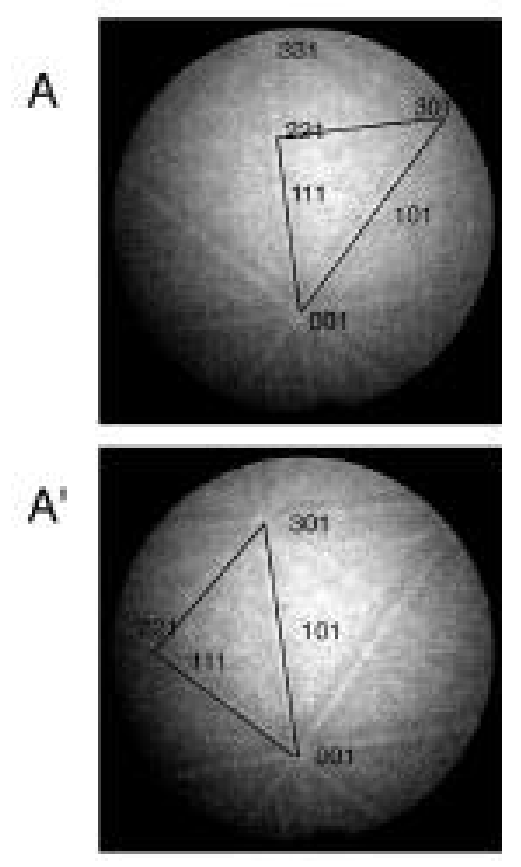

Fig. 39.

Kikuchi backscatter patterns from regions in grain $A$ and grain $A^{\prime}$. The 001 is easily recognizable by three intersecting bands of high contrast. Rotation is noted by highlighting the triangle between the [001], [221] and [301] directions; the relationship between $A-A^{\prime}$ is $\approx 43^{\circ}$ [001].

Table 4. Bicrystal grain boundaries created

\begin{tabular}{ccc}
\hline Regions & \multicolumn{1}{c}{ Actual $\theta$ /axis range } & Approximate $\theta$ and axis \\
\hline A-A' & $41^{\circ}\left[\begin{array}{llll}1 & 1 & 9\end{array}\right]-44^{\circ}\left[\begin{array}{lll}1 & 0 & 9\end{array}\right]$ & $42.5^{\circ}[001]$ \\
$2-1^{\prime}$ & $41.4^{\circ}\left[\begin{array}{lll}\overline{12} & \overline{1} & 1\end{array}\right]-42.6^{\circ}\left[\begin{array}{lll}7 & \overline{81} & 5\end{array}\right]$ & $42.0^{\circ}\left[\begin{array}{ll}100\end{array}\right]$ \\
$2-2^{\prime}$ & $47.5^{\circ}\left[\begin{array}{lllll}\overline{3} & \overline{84} & 1\end{array}\right]-49.5^{\circ}\left[\begin{array}{lll}\overline{25} & \overline{1} & 1\end{array}\right]$ & $48.5^{\circ}\left[\begin{array}{ll}100\end{array}\right]$ \\
\hline
\end{tabular}

data when viewed quantitatively. First, the absolute magnitude of $J_{c}$ measured for grain boundaries in the bulk samples is significantly lower than that measured for a thin film counterpart. Second, the functional dependence of $J_{c}$ on $\theta$ is weaker in the case of the bulk samples when compared with the thin films; the slope of $J_{c}(\theta)$ for the bulk samples is approximately half of that for the thin films. We have proposed a model wherein the pinning of vortices along the grain boundary explains the discrepancies in the data of Fig. 40. We examine here several key consequences of this model and compare predictions with experimental data and find that pinning of vortices along a grain boundary can substantially affect the $J_{c}$ measured across the boundary. This finding suggests that a complete picture of current transport across grain boundaries must consider vortex dynamics and the influence of vortex motion on measured dissipation.

Bicrystals of Y-123 were prepared for this work by our dual-seeded melt-textured growth process described previously. Transport measurements were carried out in a fouror six-terminal configuration at $77 \mathrm{~K}$. Magnetic fields $(\mathrm{H})$ up to $0.1 \mathrm{~T}$ were applied. In most cases, the field was applied with $\mathrm{H}$ parallel to the c-axes of the grains. The dependence of $\mathrm{J}_{\mathrm{c}}$ on field orientation was measured for one $90^{\circ}$ [100] tilt boundary by rotating the field in the plane of the boundary. 


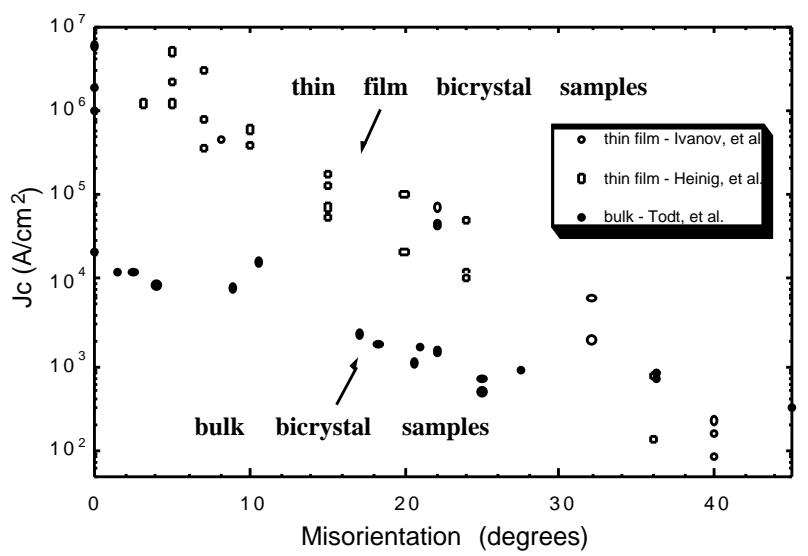

Fig. 40. Critical current density at $77 \mathrm{~K}, \mathrm{~B}=0$, as a function of $\theta$ for single-grain boundaries in thin film and bulk bicrystals

In the very-low-angle regime of Fig. 40, the differences in $J_{c}$ may be ascribed to the significantly lower $J_{c}$ in the grains on each side of the boundary in the bulk samples when compared with the relatively high $\mathrm{J}_{\mathrm{c}}$ values that are typical of high-quality thin films. Because each transport measurement of a grain boundary includes some contribution from the grains on each side owing to the placement of voltage contacts, any dissipation in the grains contributes to the measured voltage. The result is that the values measured for the grain boundary $J_{c}$ in the bulk samples are influenced by the $J_{c}$ of the grains and should, in fact, be somewhat lower if the boundary also contributes to the dissipation. However, in the higher-angle regime, $J_{c}$ values clearly reflect dissipation from the grain boundary in both the thin films and bulk bicrystals. For example, looking at the values for $\theta \approx 20^{\circ}$, we see that in both cases, the measured $J_{c}$ values are nearly one order of magnitude lower than the values for low- $\theta$, suggesting the strong influence of the boundary. In this situation, it is difficult to explain the difference in $\mathrm{I}_{\mathrm{C}}$ between the two types of boundaries.

All data in Fig. 40 are for boundaries that are nominally [001] tilt boundaries. It has been shown that thin-film boundaries are not well defined; they meander significantly, leading to a grain boundary plane that changes as a function of position. In contrast, the grain boundaries in the bulk bicrystals are highly planar. Considering this structural difference, we proposed a model based on the pinning of Josephson vortices along the boundary to explain the discrepancy in $J_{c}$ values. We assumed that the presence of Josephson vortices is a consequence of weak coupling across the boundaries, and that the Josephson critical current $J_{c J}$ follows an exponential decay with $\theta$. Under these circumstances, $J_{c}$ values measured for the thin-film boundaries should reflect the degree of pinning and scale linearly with $J_{c J}$. For bulk bicrystals, $J_{c}$ occurs once a vortex enters the boundary because it may then be driven along the boundary rather freely, leading to dissipation. In this situation, $\mathrm{J}_{\mathrm{c}}$ is expected to scale as $\mathrm{J}_{\mathrm{cJ}}{ }^{1 / 2}$.

In evaluating this model, one must consider whether the assumption that pinning of Josephson vortices based on the meandering configuration of the boundary is reasonable. The dominant contribution to the energy of a Josephson vortex is associated with the circulating screening currents. Intuitively, any redistribution of the currents required to move the vortex would be resisted. Thus, geometrical deviations of the boundary are expected to promote pinning. In addition, the grain boundary plane also changes as a function of position, and it has been speculated that the strength of coupling may be a 
function of the grain boundary. Recently, we have been able to measure the effect of the boundary plane on $\mathrm{J}_{\mathrm{c}}$ for $90^{\circ}$ [100] grain boundaries. While performing these measurements, we found a substantial difference in $J_{c}$ based on the boundary plane, with $90^{\circ}[100]$ twist boundaries supporting a $J_{\mathrm{c}}$ nearly an order of magnitude higher than their $90^{\circ}[100]$ symmetric-tilt counterparts. Thus, the idea that pinning of Josephson vortices in thin film boundaries can be strong is reasonable, given that a boundary that consists of a series of differing planes.

In contrast to the thin films, microstructural characterization of the bulk boundaries reveals no features that would be effective in pinning vortices. Under these circumstances, $\mathrm{J}_{\mathrm{c}}$ is determined once the magnetic field reaches $\mathrm{H}_{\mathrm{c} 1}$ and vortices can penetrate the boundary. Therefore, $\mathrm{I}_{\mathrm{c}}$ should scale inversely with applied magnetic field inasmuch as the total field is equal to the applied field plus the self-field generated as a result of the current passing through the sample. Figure 41 shows $\mathrm{I}_{\mathrm{c}}$ data as a function of applied field for two bulk grain boundaries. In each case, the initial decrease obeys the expected relationship. Note that $\mathrm{I}_{\mathrm{c}}(0)$ for the $25^{\circ}$ boundary is higher than that for the $18^{\circ}$ boundary and that they cross over at $\approx 5$ Gauss. This behavior is based on the sample-generated self field, which is dependent on the sample dimensions. As a further test of consistency, we can use the slope of $I_{c}(B)$ to calculate the dimensions of the sample measured and, as noted in the figure, the agreement between this calculated value and the actual dimensions of the sample is within $\approx 25 \%$, supporting the concept that $J_{c}$ is determined by flux penetration along the boundary for this orientation.

We have also examined the dependence of the critical current on the orientation of the applied magnetic field. In this case, we used a $90^{\circ}$ [100] symmetric-tilt boundary. As shown in the inset of Fig. 42, the applied field of $0.1 \mathrm{~T}$ was rotated in the plane of the boundary. The data of Fig. 42 show a smooth variation of $I_{c}$ with field orientation, with a maximum corresponding to a field that is oriented parallel to the ab planes of the grains $\left(\theta \approx 0^{\circ}, 180^{\circ}\right)$ and a minimum corresponding to a field that is oriented at $\theta \approx 90^{\circ}$. The absence of sharp peaks indicates that the planar boundaries are uniform and do not contain discrete pinning centers. The general shape of the curve is similar to that obtained for single crystals or thin films, in which a maximum in $J_{c}$ occurs when the field is oriented along the $a b$ planes and a minimum in $J_{c}$ occurs when the field is parallel to the $c$ axis. The interpretation of these results is based on strong pinning of Abrikosov vortices due to the layered structure of $\mathrm{Y}-123$ when the field is parallel to ab and relatively weaker pinning when the field is parallel to the c axis. Thus, these results suggest that even though only coreless Josephson vortices are expected along the boundary, pinning can play a role in determining $\mathrm{J}_{\mathrm{c}}$, even for these straight grain boundaries.

All of the data shown are consistent with the conclusion that vortex pinning along a boundary can contribute to the $\mathrm{J}_{\mathrm{c}}$ measured across a boundary. In very-low-angle grain boundaries, where the vortices may exist as Abrikosov vortices, pinning based on microstructural features has been shown previously. For the boundaries with greater angles that are considered here, Josephson vortices must be pinned. One aspect of pinning is that the length scale of the pinning center should be on the order of the length scale of the vortex. For Josephson vortices, the characteristic length is the Josephson penetration length $\lambda_{\vartheta}$, which is inversely proportional to the strength of coupling for the junction; therefore, it is expected to vary as a function of $\theta$. However, $\lambda_{\vartheta}$ is expected to be rather long for the higher-angle boundaries considered here. This length scale is 


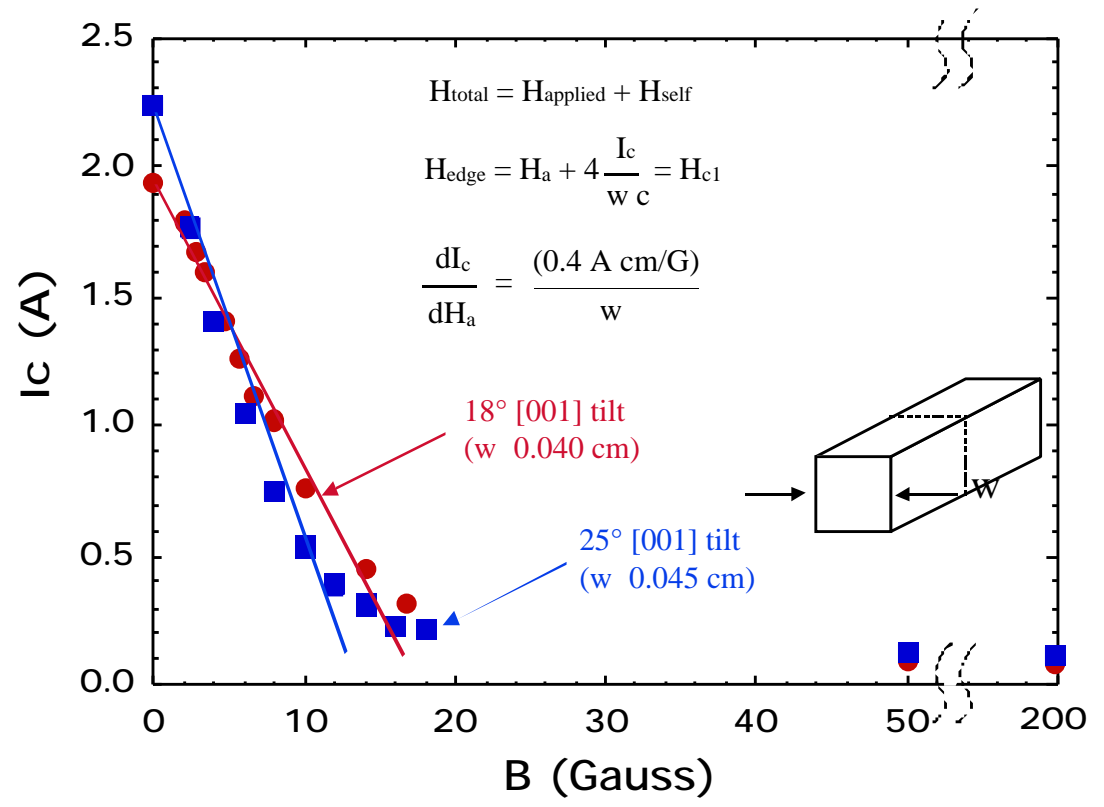

Fig. 41. $\mathrm{I}_{\mathrm{C}}$ at $77 \mathrm{~K}$ as a function of applied magnetic field; applied field was parallel to c-axis of each grain.

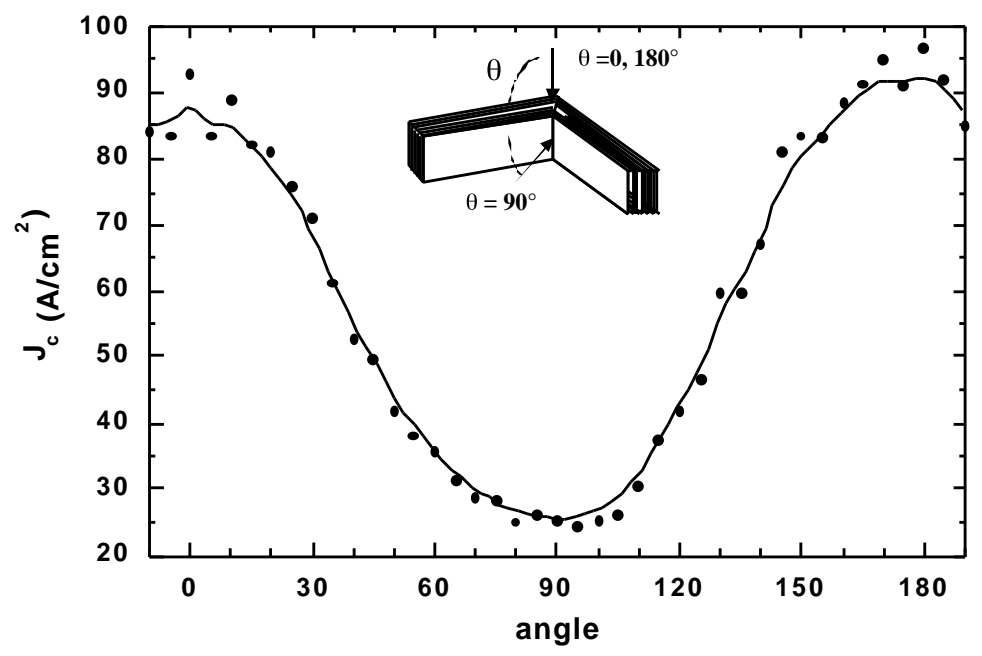

Fig. 42. $\mathrm{J}_{\mathrm{c}}$ at $77 \mathrm{~K}$ as a function of applied magnetic field; applied field of $0.1 \mathrm{~T}$ was oriented in plane of boundary as shown in inset. 
consistent with the characteristic period of meanders in thin film boundaries, which, in that case, would lead to relatively strong pinning. In the bulk grain boundaries, however, no features are found along the boundary on this length scale and consequently pinning is expected to be weak.

However, the data of Fig. 42 suggest that the motion of Josephson vortices can be impeded in certain directions. The maxima in $J_{c}$ correspond to field orientations for which the vortices must move across the ab planes of the sample. The dependence of $J_{c}$ on angle can be explained in terms of the drag force induced by the intrinsic anisotropy of the Y-123 grains, as shown in Fig. 43. The circulating currents that define the Josephson vortex pass through the grains that consist of superconducting and insulating layers. In an idealized system, the currents will be passed as supercurrent and Josephson current in each of the layers, respectively. In this highly overdamped system, viscous drag is expected to impose a resistance to motion in the direction of the layering. Note that motion parallel to the layers is not subject to this friction and thus is expected to be free of pinning, as in the case of the [001] tilt boundaries discussed previously.

Finally, it must be pointed out that we consider the presence of vortices and their motion to be a key aspect of dissipation. In this respect, we consider only boundaries that are sufficiently long to contain Josephson vortices (long-junction limit). In many nondevice applications (practical conductors, for example), most boundaries are expected to satisfy this condition. However, the intrinsic conduction across a short length of the boundary is also an essential element. In this model, we do not consider the precise nature of conduction across a short-length junction or how it varies as a function of $\theta$. Our model assumes some fundamental relationship between the Josephson critical current $J_{c J}$ and $\theta$ and uses that relationship, together with vortex motion, to understand the relationship between the measured critical current $J_{c}$ and $\theta$.

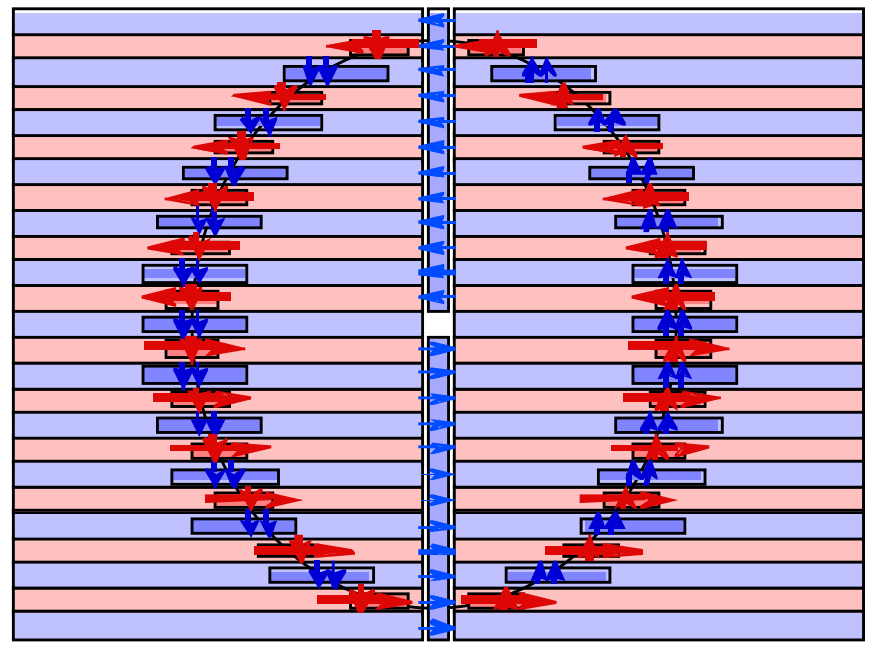

Fig. 43. Representation of J osephson vortex at a grain boundary. Layering in grains represents superconducting and J osephson components of circulating currents. Because of anisotropy, vortex motion along boundary in the direction perpendicular to the page is not resisted, but motion up or down must overcome strong drag resistance. 


\subsubsection{Melt-Processed Y-123}

We have continued our collaboration with Dr. Pavel Diko of the Institute of Experimental Physics in Slovakia to examine processing, microstructural development, and properties of melt-processed Y-123 composites that contain $\mathrm{Y}_{2} \mathrm{BaCuO}_{5}(\mathrm{Y}-211)$. As described above, most of the bulk materials have been processed at ANL by our proprietary seeding method. Some were obtained from Superconductive Components, Inc. (Columbus, $\mathrm{OH})$. Microstructures were studied by optical and electron microscopy. The goals of this work were to identify features that can perhaps be manipulated to produce superior properties in future materials and to characterize and manipulate mechanical properties of these ceramics.

Y-123 Melt Growth. Increased understanding of the growth mechanisms of bulk melt-processed $\mathrm{Y}-123 / \mathrm{Y}-211$ superconductors has led to improved thermal treatments that can enhance transport and levitation properties. Melt-processing of $\mathrm{Y}-123 / \mathrm{Y}-211$ samples requires heating above the peritectic melting point. At this temperature, samples retain their original shape. To sustain growth of $\mathrm{Y}-123$ grains during cooling, dissolution of $\mathrm{Y}-211$ particles in the melt is essential to supply $\mathrm{Y}^{+3}$ ions to the solidification front. When the Y-123 solidification front encounters a Y-211 particle, it will either push it along or engulf it, depending on the Y-211 particle size, the growth-front velocity, and the various interfacial energies.

Y-123 grains grow with $\{100\}$ facets. We have shown that the growth velocity in the a- and c-directions depends on solidification conditions such as undercooling and temperature gradients. Growth in these two specific directions causes the a- and c-grown parts of one Y-123 grain to be divided by surfaces that are close to $\{110\}$-type planes. We report here an observed macroscopic change in sample shape during solidification and relate this change to the growth mechanism of Y-123 from the Y-123/Y-211 mixture. Five-domain Y-123/Y-2 11 bulk samples were produced with cubic, five-domain Nd-123 crystal seeds. Study of such samples has helped to elucidate grown mechanisms of melt-processed Y-123.

The macrostructure of the top surface of the five-domain sample can be seen in Fig. 44. Macrocracks parallel to the $\mathrm{a}-\mathrm{b}$ plane indicate the $\mathrm{c}-\mathrm{axis}$ orientation and the boundaries between the four side domains. The darker bands along each side of the $90^{\circ}$ boundaries are regions of higher Y-211 density. These samples exhibit an interesting shape change. Figure $44 \mathrm{~b}$ clearly shows that an originally circular shape became deformed during solidification. The c-growth parts of the sample bulged and the a-growth parts contracted. This change of shape was caused by macroscopic mass transport associated with the solidification process. Such a change of shape has not been observed in single-domain samples in which flat, single-domain Nd-123 seeds were used.

Y-123 grains in the form of a parallelepiped grew from the Y-2 $11+$ melt mixture, with growth-front surfaces perpendicular to the $\mathrm{c}-$ and a- axes. In Fig. 45, the situation at the edge of a growing Y-123 grain is depicted for the case in which the growth rate is faster along the c-axis than along the a-axis, as is observed in these samples. A layer with an $\mathrm{Y}$-concentration gradient of thickness $\mathrm{d}$ is formed at the growth front because $\mathrm{Y}$ is consumed by the growing grain. In this layer, $\mathrm{Y}-211$ particles dissolve to provide $\mathrm{Y}$ for the formation of solid Y-123. At the edges, ions in the melt should be distributed to a-a and 

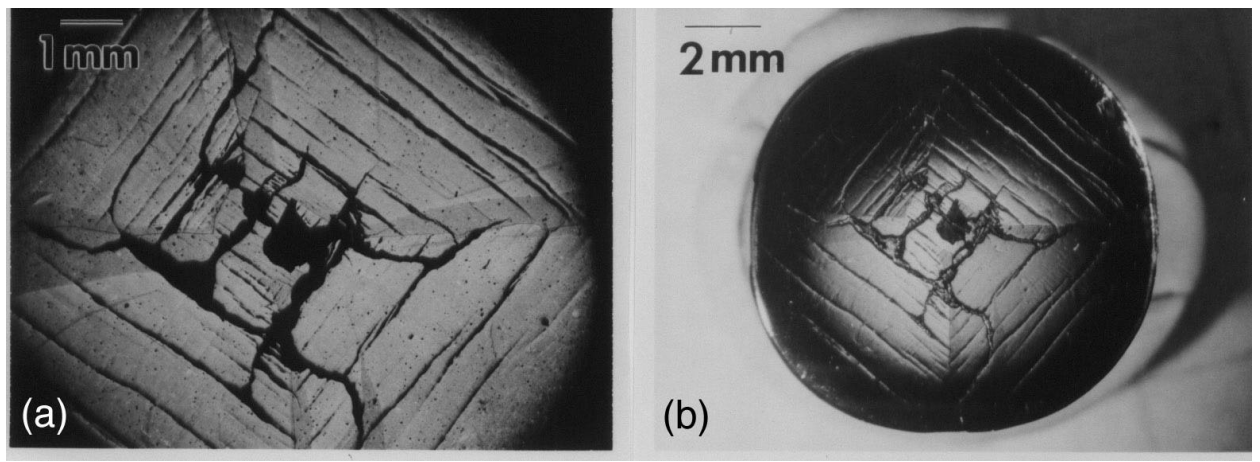

Fig. 44. Top surface of five-domain $Y-123 / Y-211$ pellet: (a) polarized-light photograph showing cracks and domain boundaries and (b) photograph showing distortion from originally round shape.

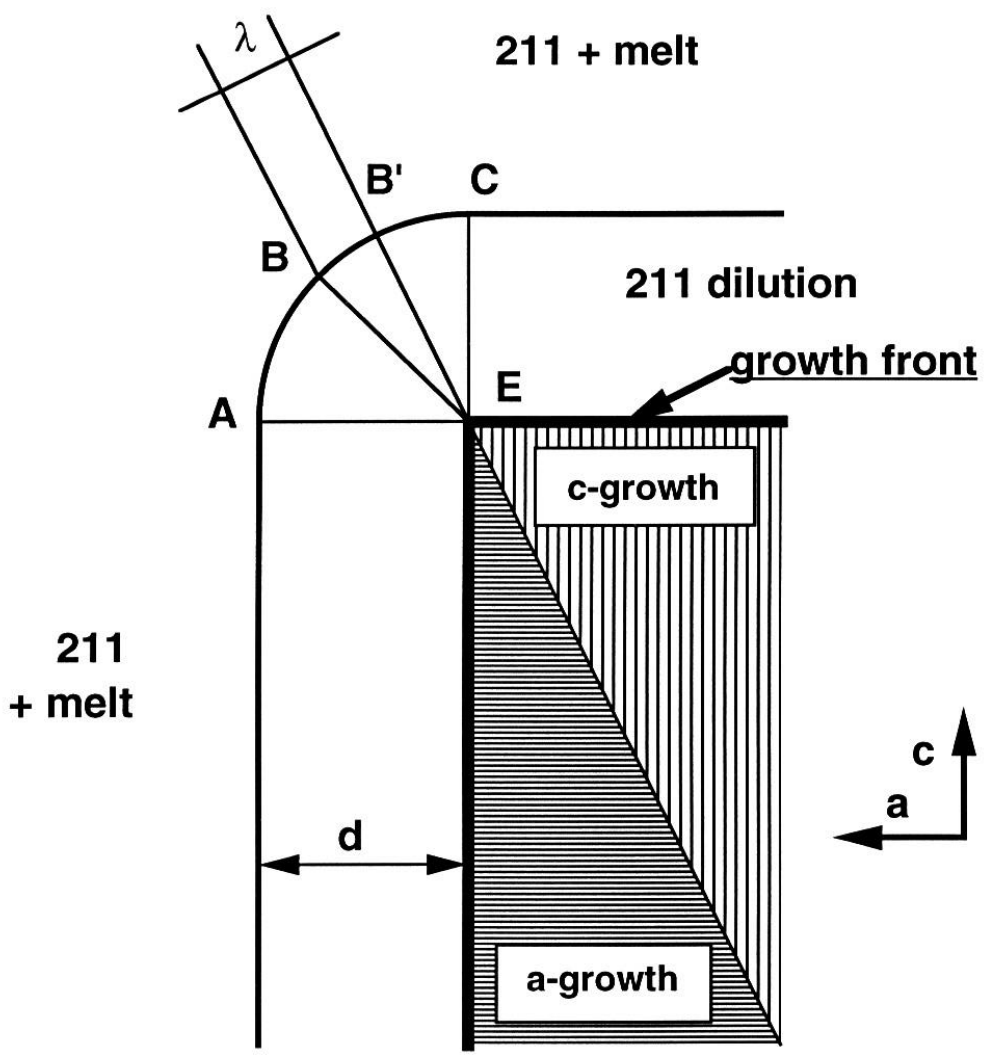

Fig. 45. Schematic diagram of edge between a- and c-growth regions of $Y-123$ grain for case in which c-growth is faster. 
a-c surfaces. The area defined by EBB' can be related to macroscopic mass transport caused by the difference in the rate of a- and c-growth. A melt layer of the thickness $\lambda$ moves from the a-growth to the c-growth region because of the more rapid c-axis growth.

Excess liquid in front of the c-growth part of the crystal could perhaps be removed by flow through the regions of $\mathrm{Y}-211+$ melt that are outside of Layer $\mathrm{d}$ adjacent to the growth front. This flow is apparently minimal, however, because of the very high concentration of Y-211 particles $(\approx 50 \mathrm{wt} . \%)$ in the region outside Layer d. The high Y-211 concentration acts to impede liquid flow outside Layer d. The lower concentration of Y-211 and the attendant lower viscosity within Layer $d$ will, however, allow inflow of the excess liquid from the edge. This flow, caused by anisotropy of growth velocities, induces pressure in Layer $d$ at the c-growth part of the solidifying $\mathrm{Y}-123$ grain. This pressure is relaxed by mechanical deformation of the Y-211 + melt mixture, leading to bulging of the sample in regions formed by the c-axis growth.

This process has been modeled. The model qualitatively predicts the shape change and Y-211 concentration profiles that are observed in bulk, five-domain $\mathrm{Y}-123 / \mathrm{Y}-211$ superconductors. It also sheds light on the role of $Y$ diffusion in microstructural development.

Mechanical Properties of Melt-Processed Y-123. Melt-processed Y-123/Y-211 superconductors are being developed for many applications in which magnetic fields will be high. Resultant mechanical stresses can perhaps be sufficiently large to induce gross fracturing of the superconductor. It may well be that mechanical considerations surpass superconducting consideration in component design.

The microstructures of melt-processed Y-123/Y-211 materials, including the single-domain materials, are complex. Subgrain boundaries, Y-211 gradients, and retained phases from the melt are common. Although much has been written about the basic mechanical properties of Y-123, little emphasis has been placed on mechanical properties with respect to variations in local microstructure or processing. We report here on measurements of mechanical properties of melt-processed Y-123/Y-211 and efforts to improve them through heat treatment.

To produce $\mathrm{Y}-123 / \mathrm{Y}-211$ compacts, a mixture of composition $\approx 74$ wt.\% Y-123, 25 wt.\% Y-211, and 1 wt.\% $\mathrm{PtO}_{2}$ was prepared. Hexagonal pellets were pressed in a 25-mm-wide die and processed in air by conventional means. A Nd-123 single-crystal seed was placed on the center of the top surface of each pellet to promote single-domain growth.

Specimens for mechanical studies were generally cooled in $\mathrm{N}_{2}$. The specimens were annealed by one of two schedules: heated in $\mathrm{O}_{2}$ to $\approx 450^{\circ} \mathrm{C}$ and held for an extended period; or heated in $\mathrm{N}_{2}$ to $\approx 720^{\circ} \mathrm{C}$, and then annealed stepwise in $\mathrm{O}_{2}$. The stepwise annealing was designed to take advantage of the more-rapid oxygenation kinetics and to minimize microcracking in the specimens. We thought that by increasing the oxygen content slowly, and thus changing the lattice parameters of the Y-123 gradually, cracking might be minimized.

Although the melt-processed Y-123/Y-2 11 compacts were nominally single-domain, they exhibited subgrain boundaries caused by growth along two types of fronts: c-axis growth below the single-crystal seed and a-axis growth from the four sides of the seed 
(Figs. 46a and b). More important, the distribution of Y-211 particles in the compacts was not homogeneous.

The most prominent inhomogeneity was an increase in Y-211 density along the c-growth direction (Fig. 46c). Another Y-2 11 inhomogeneity developed at $90^{\circ}$ subgrain boundaries between a- and c-axis growth fronts. Bands of higher Y-211 concentration ran across both sides of the boundaries (Fig. 47).

We attempted to measure elastic modulus, hardness, and flexural strength vs. location within the Y-123/Y-211 compacts. Elastic modulus could not be measured because good ultrasonic signals could not be obtained. Scattering from second phases and cracks was too severe. Hardness depended on whether the indentation was parallel or perpendicular to the c-axis of the compact. Hardness also exhibited a pattern with respect to location, but not with respect to annealing schedule. In brief, the Vickers hardness results were:

1. Hardness was generally $\approx 20 \%$ higher when measured perpendicular to the c-axis rather than parallel to the c-axis.

2. The minimum hardness of 3.3-3.7 GPa was found beneath the seed.

3. The maximum hardness of 5.4-6.1 GPa was found a few millimeters from the rim, near the top of the compact.

4. In the bottom halves of the compacts, hardness was 4.2-5.3 GPa, regardless of position.
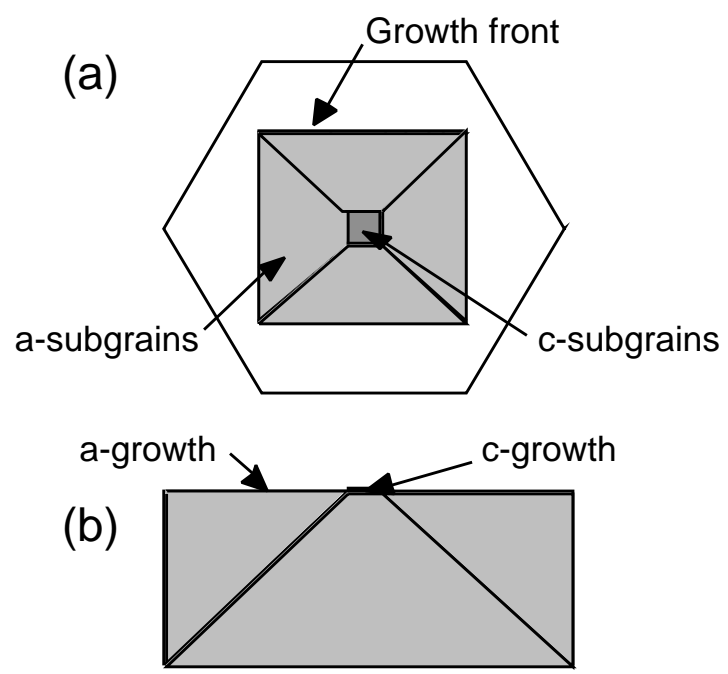

(c)

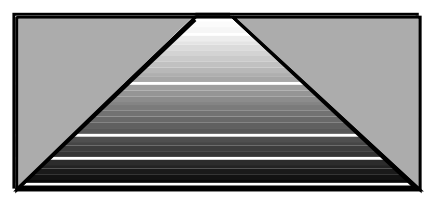

Fig. 46. Schematic diagram of (a) and (b) crystal growth directions, and (c) $Y-211$ distributions in single-domain Y-123/ Y-211 compacts. 


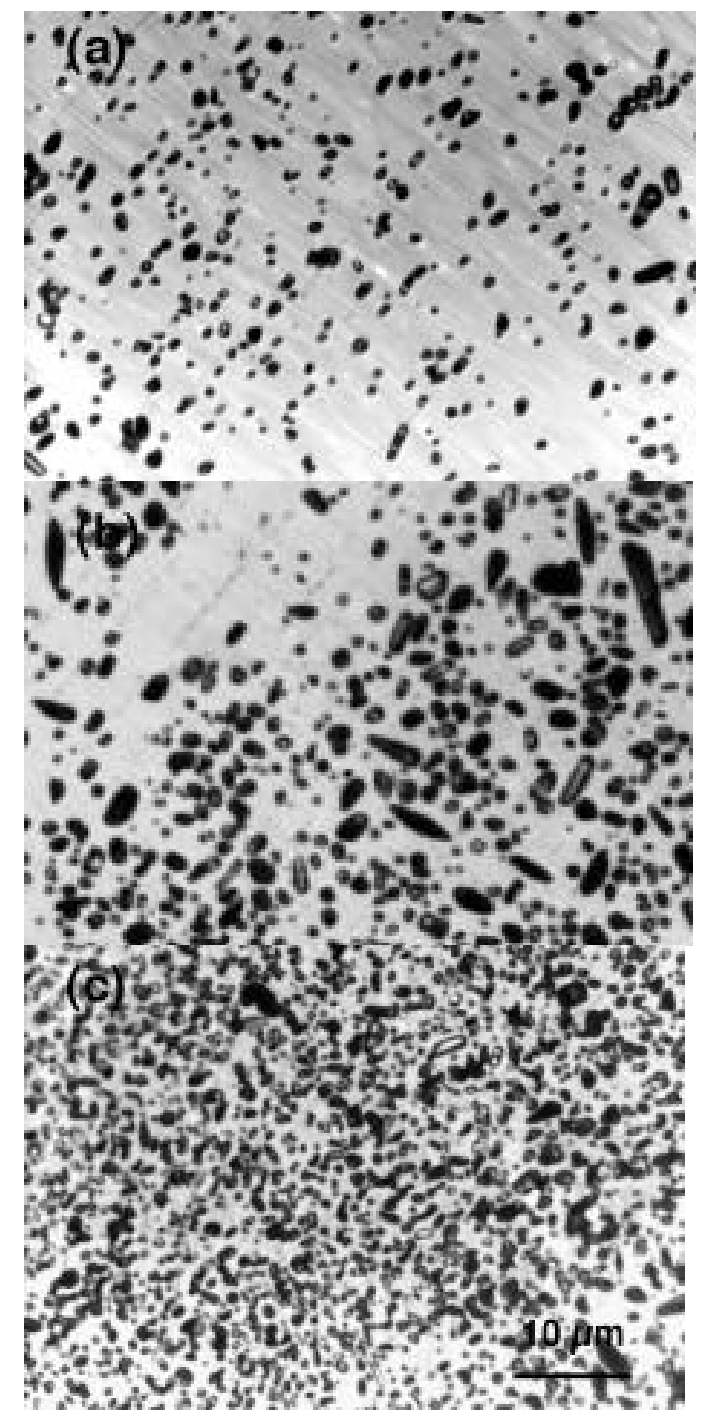

Fig. 47.

Optical photomicrographs showing $\mathrm{Y}-211$ concentrations: (a) region beneath the seed, (b) c-growth region near compact perimeter, and (c) bands of higher $\mathrm{Y}-211$ concentration along inner side of $a-c$ subgrain boundaries.

Low Vickers hardnesses correlated with low concentrations of the Y-211 phase. We attempted to estimate fracture toughness from cracks that emanated from the hardness impressions, but the cracks tended to follow crystallographic planes and were strongly influenced by defects; therefore, no reliable fracture-toughness data could be obtained. Average strength values with position are shown in Fig. 48.

The strength values were similar to those reported for melt-processed Y-123 [23]. In general, highest strengths were in the center, and the tops of compacts were stronger than the bottoms. The fact that strength could not be improved through annealing is perhaps not surprising. It has been shown that cracks tend to form at Y-211 particles during cooling because of differences in thermal expansion between Y-123 and Y-211. Although Y-211 within a Y-123 matrix can lead to improved fracture toughness [13], strength does not follow suit because of the introduction of a new population of significant flaws. Y-211 particles within Y-123 grains are necessary for optimal superconducting properties. However, our results indicate adverse effects of Y-211 on strength. 


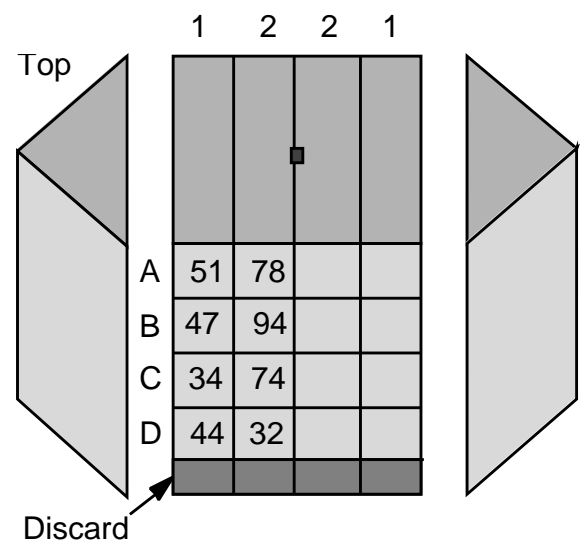

Fig. 48.

Schematic diagram of positions of bending bars, and corresponding flexural strengths in MPa for $\mathrm{Y}-123 / \mathrm{Y}-211$ compacts.

\subsection{Applications and Devices}

Successful design of devices requires understanding of the relations between structure and properties and among properties. Magnetic properties are of particular interest to applications such as flywheels. We report here on investigation of the forces between a permanent magnet and an HTS and on the phenomenon of magnetic diffusion.

\subsubsection{Forces between a Permanent Magnet and a High-Temperature Superconductor}

The interaction between a permanent magnet (PM) and an HTS has been a subject of considerable interest for the last decade. Applications of HTSs to flywheel and magnetic-bearing systems are based on the high pinning forces between HTSs and PMs, which are essential for lateral stability. We have investigated both vertical and lateral force components, including their respective stiffnesses under various cooling conditions, and we have compared our experimental results with analytically calculated forces and respective stiffnesses that are based on a frozen-image method.

The experimental apparatus consisted of a cylindrical NdFeB PM attached to an $\mathrm{x}-$ and $z$-axis strain-gauge force transducer, mounted on hand-controlled micrometer stages that provide vertical $(z)$ and horizontal $(x)$ movement at low velocity. The PM disk was polarized axially with a magnetization of $\mu_{0} \mathrm{M}=0.831 \mathrm{~T}$. A 6.5-mm-high, 12.7-mm-diameter steel keeper immediately above the PM was mechanically connected to the force transducer. Two melt-textured, single-domain, bulk Y-123 HTSs were used; each was $\approx 30 \mathrm{~mm}$ in diameter and $12 \mathrm{~mm}$ thick.

The force and stiffness measurements were performed in zero-field cooling (ZFC) and field cooling (FC). To avoid interactions with the liquid $\mathrm{N}_{2}$, the closest distance between the PM and HTS was $\approx 0.5 \mathrm{~mm}$. Calculations included changes in magnetization of the PM with temperature.

To prevent large temperature fluctuations, we installed a bifilar winding with a Nichrome resistance wire around the PM to keep the PM temperature at $\approx-10^{\circ} \mathrm{C}$. With this winding, the resulting magnetic field was $\approx 0 \mathrm{G}$, which was negligible when compared with the field from the PM. 
To measure rotational losses of a levitated NdFeB PM disk rotor over a bulk HTS, the $\mathrm{PM}$ was in a vacuum of $<10^{-6}$ torr. The PM rotation was accelerated and decelerated by a cold gaseous nitrogen jet impinging approximately tangentially on the PM perimeter from a small-diameter copper tube. Once the PM reached the desired speed, the gas jet was shut off. The rotation of the PM was monitored by a tachometer. The PM was allowed to freely spin down for 3-5 min after acceleration and speeds were recorded every $5 \mathrm{~s}$. When we were interested in details of resonance behavior, we recorded data every second. At intervals throughout the experiment, the height of the levitated PM was measured with a traveling telescope; no change in height from the initial levitation value was observed to within $10 \mu \mathrm{m}$.

Vertical force vs. vertical distance measured for cooling height $(\mathrm{CH})=30 \mu \mathrm{m}$ (i.e., ZFC) is shown in Fig. 49, with a descending vertical traverse to $1 \mathrm{~mm}$, followed by an ascending vertical traverse to $30 \mathrm{~mm}$. Maximum levitation force was $22.4 \mathrm{~N}$, with a corresponding levitation pressure of $177 \mathrm{kPa}$. The small hysteresis indicates that at this $\mathrm{CH}$, the HTS would behave mostly as a diamagnet. Such behavior is typical of good melt-textured Y-123 in ZFC. The HTS was again cooled with $\mathrm{CH}=0.5 \mathrm{~mm}$ (i.e., $\mathrm{FC}$ ), and the vertical force versus vertical distance was measured during an ascending traverse out to $25 \mathrm{~mm}$, followed by a descent to $0.5 \mathrm{~mm}$, as seen in Fig. 50. Compared to that in Fig. 49, the hysteresis loop is much larger in Fig. 50, indicating that flux pinning should play a more dominant role when the $\mathrm{CH}$ is lower. This would be expected, even if $\mathrm{J}_{\mathrm{c}}$ were to be independent of magnetic field and flux pinning was equally strong for all PM heights.

In a second series of measurements, the HTS was cooled with the PM at a given $\mathrm{CH}$, then the PM was moved vertically to a given measurement height $(\mathrm{MH})$, after which the vertical and horizontal forces were measured in several horizontal traverses. The results are shown in Figs. 51 and 52. The measurements at large $\mathrm{CH}$ showed a new behavior. When the magnet moved laterally relative to the center of the superconductor, the vertical force decreased because of the edge effect of the HTS. For small $\mathrm{CH}(\leq 7 \mathrm{~mm})$, when the magnet moved laterally relative to the center of the HTS, the vertical force increased. At large $\mathrm{CH}(>15 \mathrm{~mm})$ when the PM moved away from the center of the HTS, the lateral force increased, indicating a repulsive force. At small $\mathrm{CH}(\leq 7 \mathrm{~mm})$, when the PM moved

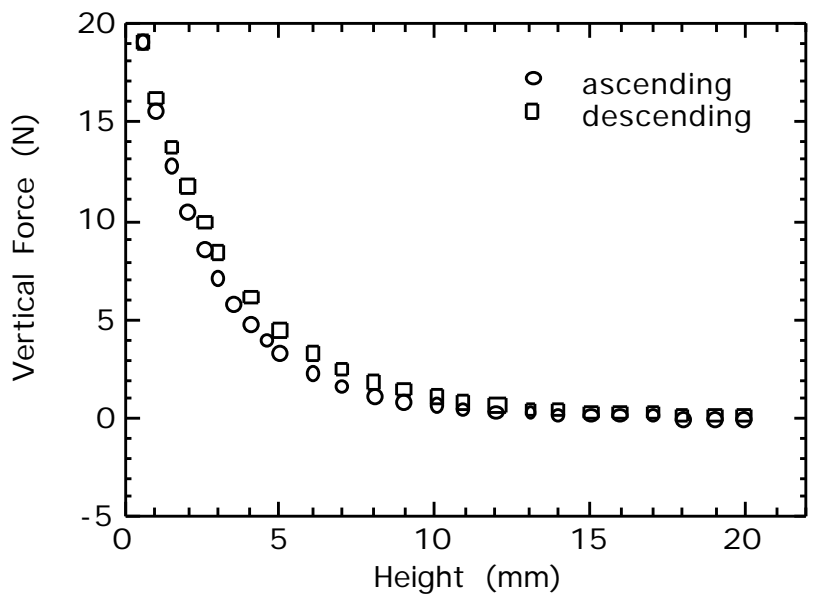

Fig. 49. Vertical force vs. height for HTS with $\mathrm{CH}=30 \mathrm{~mm}$. 


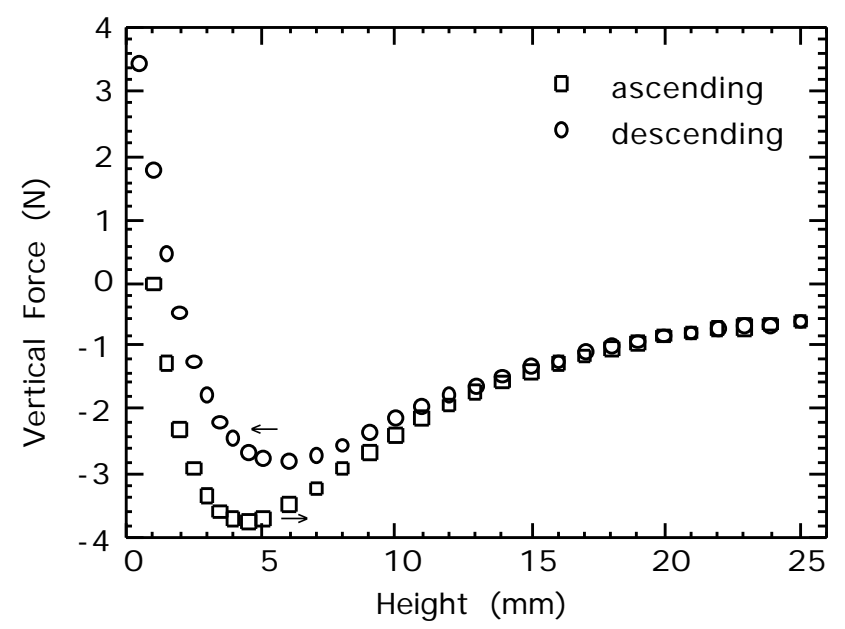

Fig. 50. Vertical force vs. height for HTS with $\mathrm{CH}=0.5 \mathrm{~mm}$.

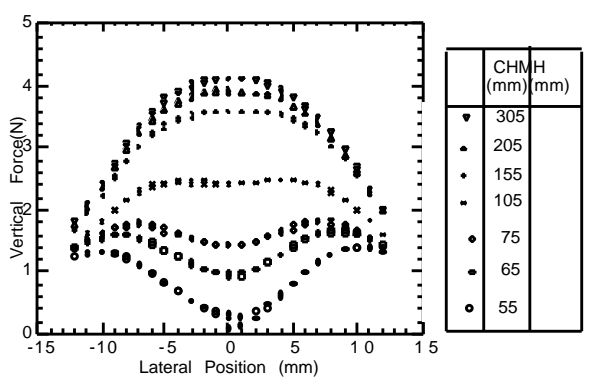

Fig. 51.

Vertical force vs. Iateral position for $\mathrm{HTS}$ at $\mathrm{MH}=5 \mathrm{~mm}$ for various $\mathrm{CHs}$.

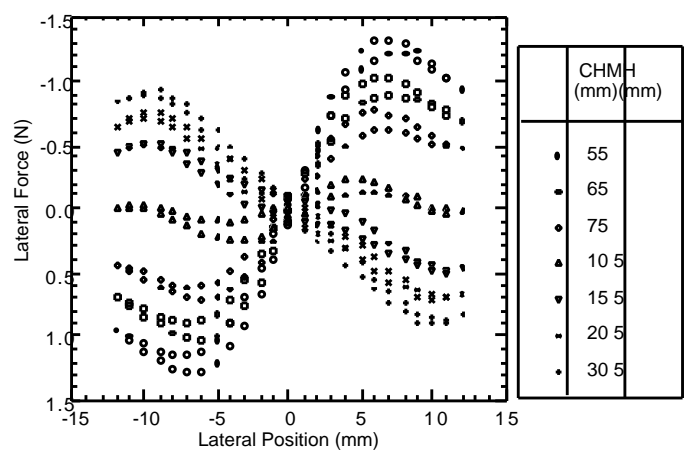

Fig. 52.

Lateral force vs. lateral position for HTS at $\mathrm{MH}=5 \mathrm{~mm}$ for various $\mathrm{CHS}$.

laterally relative to the center of the HTS, the lateral force increased from zero but was then attractive. Such behavior leads to levitational stability. The transition from large $\mathrm{CH}$ to small $\mathrm{CH}$ showed that the cooling history determined the HTS behavior.

Vertical stiffness values were determined by producing minor hysteresis loops at certain heights, first moving in a direction opposite that of the major loop from this height, with a small increment, and then returning to the same height while the force vs. 
position was measured at the same time. To a first approximation, the results show that for a given height in ZFC, the vertical stiffness was independent of the ascent or descent. On closer inspection, the vertical stiffness is always slightly greater in the first descent than in the first ascent and second descent, and the stiffness in the second descent is between that of the first descent and the first ascent in ZFC. We attribute these slight differences to differing amounts of trapped flux in the HTS.

The existence of cross-stiffness $\mathrm{K}_{\mathrm{ZX}}$ leads to a double-valued function for the radial amplitude of a levitated PM near the radial resonance (Fig. 53). The coefficient of friction is clearly double-valued in the frequency range from 5.35 to $5.55 \mathrm{~Hz}$, just below the resonant frequency. The coefficient of friction is significantly higher on the branch that begins above the resonance. The result of higher amplitudes is a larger hysteresis loss in the HTS.

In summary, forces on a PM from an HTS are primarily determined by diamagnetism and flux pinning in the HTS. To model the PM/HTS interaction in ZFC and FC, we applied the frozen-image method. This model includes no free parameters. The forces may be derived from measured properties of the permanent magnet. The frozen-image model is not expected to be useful in situations where hysteresis effects are significant, but should be useful in many practical applications. Details of the model will be published later this year. As shown in Fig. 54, agreement between the data and the model was good.

\subsubsection{Magnetic Diffusion in Superconductors}

In the practical applications of bulk HTSs, devices are often subjected to an alternating current or magnetic field at a 50-60 Hz. A powerful tool for analyzing the macroscopic response of the superconductor is Bean's critical state model. However, this is an equilibrium (steady-state) model and its applicability is limited to low frequencies. For HTSs at $50-60 \mathrm{~Hz}$, there is evidence that steady state is not reached. To explain experimental observations, one must include the effect of magnetic diffusion in the superconductor.

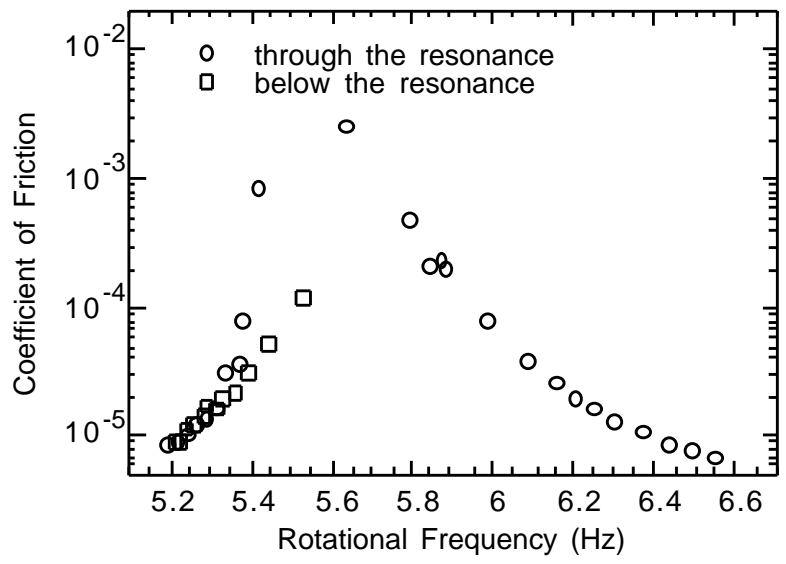

Fig. 53.

Coefficient of friction vs. rotational frequency through and below resonance. 


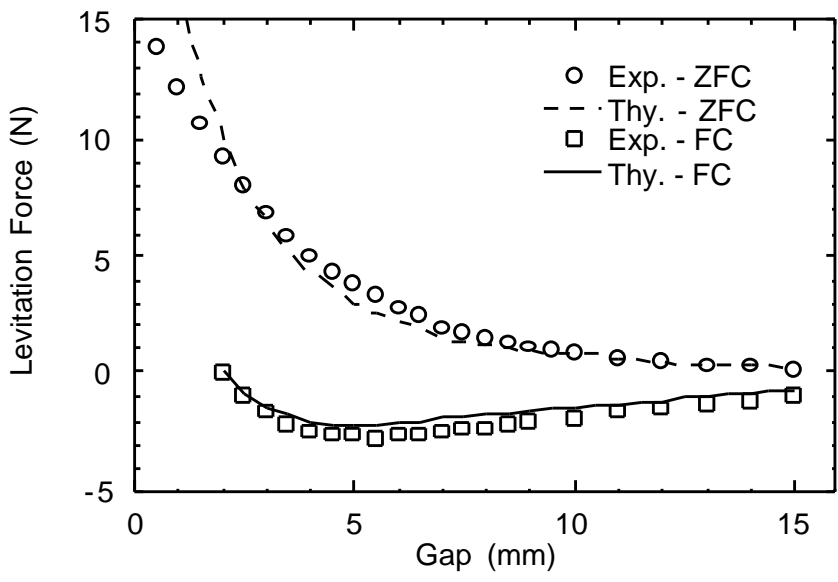

Fig. 54.

Theoretical calculations and experimental values of vertical force vs. vertical distance for ZFC and FC conditions.

Normal conductors obey Ohm's law. Assuming low frequency, the magnetoquasistatic form of maxwell's equations can be used to find a single expression describing the time evolution of the magnetic field inside an ohmic conductor. If the conductivity and the permeability are space independent, a linear magnetic-diffusion equation results.

Instead of Ohm's law, HTSs usually follow a power law,

$$
\mathrm{E} / \mathrm{E}_{\mathrm{c}}=\left(\mathrm{J} / \mathrm{J}_{\mathrm{C}}\right)^{\mathrm{n}}
$$

where $E$ is the electric field strength, $J$ is the current density, $J_{c}$ is the critical current density, $\mathrm{E}_{\mathrm{c}}$ is the electric field strength at $\mathrm{J}_{\mathrm{c}}$, and $\mathrm{n}$ is the exponent that can vary widely for different types of superconductors and depends on the pinning strength of the superconductor. If $\mathrm{n}=1$, Eq. 1 reduces to Ohm's law, the superconductor becomes normal, and there is no pinning. When there is very strong pinning, $n$ becomes very large and $J \cong J_{\text {c }}$. This is the Bean's critical state model. For $1<\mathrm{n}<\infty$, Eq. 1 describes flux creep. For superconductors with cylindrical geometry, it has been shown that the calculated current and flux distribution is close to Bean's model when $n=51$. Most bulk HTSs have values of $n<50$, and Bean's limit is not approached. In general, the power law describes the E-J characteristics of the superconductor from the flux-creep regime to the flux-flow regime. Equation 1 looks simple, but $\mathrm{n}$ is a function of both the local magnetic flux density $\mathrm{B}$ and temperature $\mathrm{T}$. We have shown that the magnetic-diffusion equation also becomes nonlinear as a result of the nonlinear relationship between $\mathrm{E}$ and $\mathrm{J}$ given by Eq. 1 .

The approach taken to obtain an appropriate expression for magnetic diffusion began by employing the magnetoquasistatic form of Maxwell equations,

$$
\begin{aligned}
& \nabla \times \mathbf{B}=\mu_{\mathrm{o}} \mathbf{J} \\
& \nabla \times \mathbf{E}=-\partial \mathbf{B} / \partial \mathrm{t} \\
& \nabla \cdot \mathbf{B}=0,
\end{aligned}
$$

where $\mu_{o}$ is the permeability of free space, $t$ is time, and $\mathbf{B}$ is the magnetic flux density (bold letters indicate vectors). The result was

$$
\nabla \times(\rho \nabla \times \mathbf{B})=-\mu_{\mathrm{o}} \partial \mathbf{B} / \partial \mathrm{t} .
$$


Equation 5 is the nonlinear magnetic-diffusion equation for superconductors that obey the power law.

We have also examined the time evolution of the magnetic flux density in a cylindrical geometry with azimuthal and axial symmetry $(\partial / \partial \theta=0$ and $\partial / \partial z=0)$.

We believe that it is worthwhile to comment on the applicability and validity of Bean's critical-state model because it is such a useful and powerful tool. First, as stated, Bean's model is valid only if the superconductor has very strong pinning (the exponent $\mathrm{n}$ is very large compared to 1). A number of HTSs, particularly for large-scale applications, may not have strong enough pinning to satisfy Bean's model. Second, as discussed in the FY 1998 Annual Report, Bean's model is valid only if the characteristic time for magnetic diffusion is much shorter than the characteristic time of the system. How fast the magnetic flux profile approaches the Bean limit depends on the exponent $\mathrm{n}$ and the rate of change of the applied field. We have shown that resistivity increases with the exponent $n$, which means that the magnetic-diffusion coefficient increases with $\mathrm{n}$ also. The larger the $\mathrm{n}$, the faster the rate of magnetic diffusion. If the rate of magnetic diffusion is high compared to the rate of change of the applied field, then Bean's model should provide a fairly accurate description of the current and magnetic-flux profiles. Otherwise, the time evolution of the flux profile must be calculated from our model.

To test our model, we conducted pulsed-current experiments (Fig. 55) on a melt-cast Bi-2212 tube and a melt-textured YBCO pellet. We found that magnetic diffusion was important for both transient experiments. The speed of magnetic flux diffusion depended strongly on the local resistivity $\rho$ in the superconductor. During a transient, the local gradient of magnetic flux density could be larger than that of Bean's model. This is particularly important because it significantly affects the local current density and resistivity. When local $\rho$ increases, so does dissipation $\left(\rho \mathrm{J}^{2}\right)$. If heat is not removed fast enough from the superconductor, the local temperature will rise. When temperature rises, the resistivity will increase, which further increases the magnetic diffusion coefficient and speeds up the magnetic-diffusion process. Iterative solutions of heat and magnetic diffusion are required and have been obtained.

In summary, high- $\mathrm{T}_{\mathrm{c}}$ superconductors usually obey a so-called power law. It was shown that the power law resulted in a nonlinear magnetic-diffusion equation because resistivity is a function of current density. It was pointed out that under transient conditions, local current density can exceed the critical current density and resistivity is increased rapidly which will (a) increase the rate of magnetic diffusion and (b) increase dissipation and heating in the superconductor. Temperature increases in the superconductor will also speed up magnetic diffusion because resistivity increases with temperature.

In general, to accurately calculate the magnetic flux density and current distributions in a superconductor subjected to a time-dependent applied field, we must solve simultaneously the magnetic-diffusion equation and the thermal-diffusion equation with appropriate initial and boundary conditions. This will require an experimentally determined E-J curve like that shown in Fig. 56, not only at $77 \mathrm{~K}$ but also at other temperatures higher than $77 \mathrm{~K}$ (at least up to the critical temperature). This is probably true for most practical applications, including a superconductor shielded core reactor and flux trapping with a pulsed-current source. If the applied field changes slowly, heating 


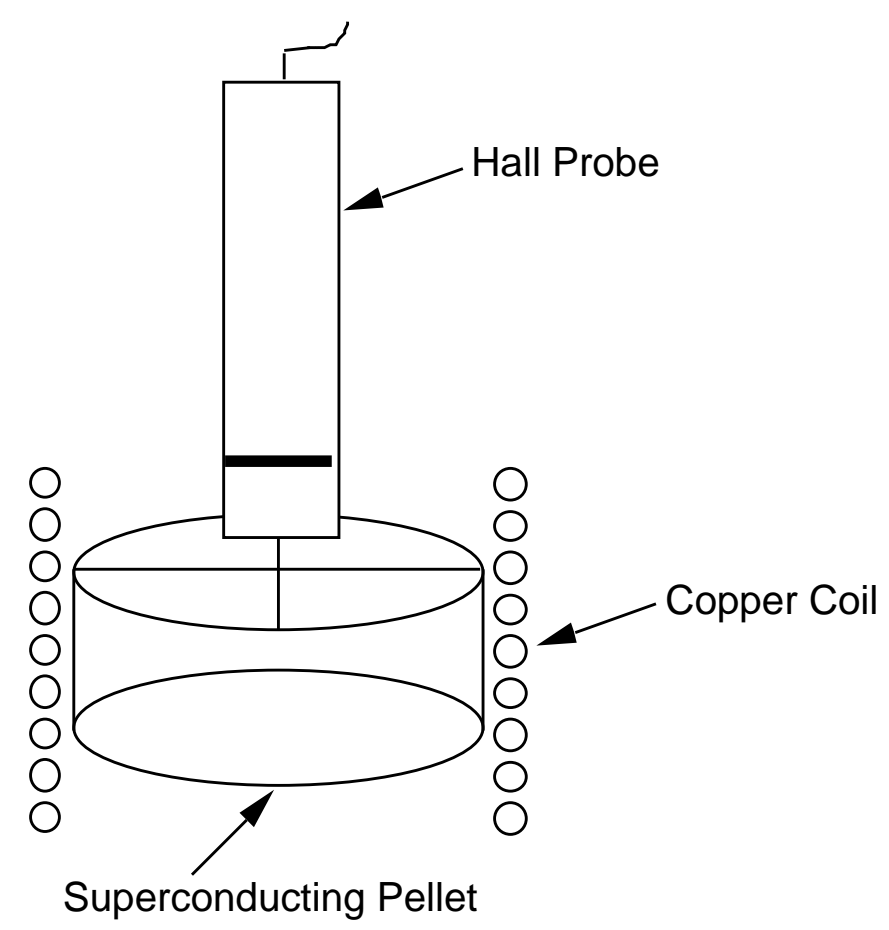

Fig. 55. Schematic diagram of superconductor pellet, copper coil, and Hall probe used in pulsed-current experiment.

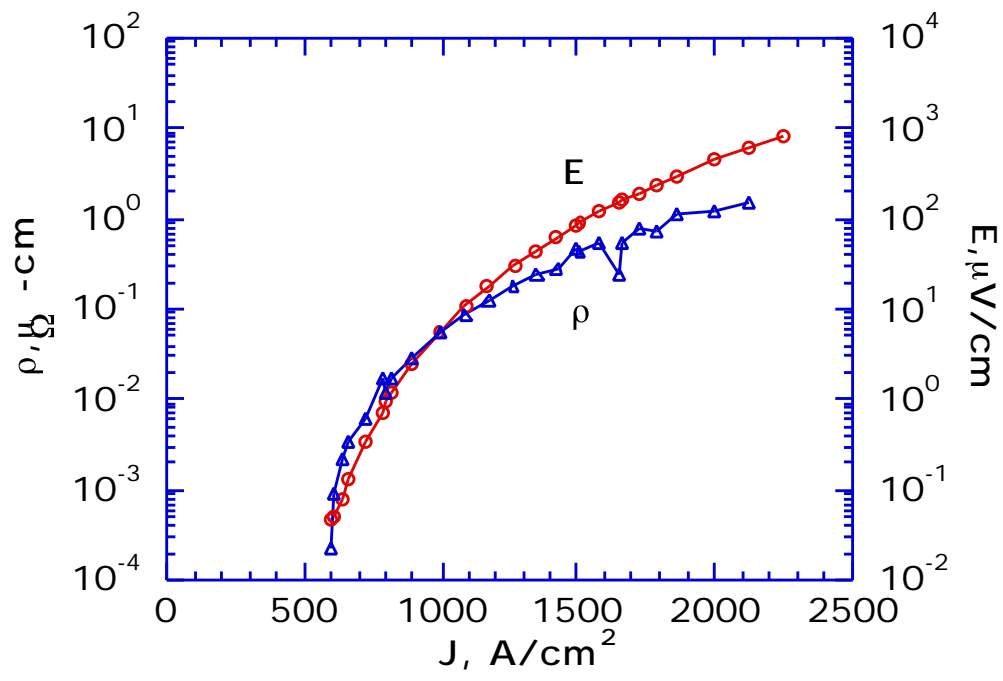

Fig. 56. Variations of $\mathrm{E}$ and $\rho$ with $\mathrm{J}$ of a Bi-2212 cylindrical rod at $77 \mathrm{~K}$ and zero applied field. 
rate is low, and sufficient time is allowed for removing the heat generated in the superconductor, then the magnetic-diffusion equation can be decoupled from the thermal-diffusion equation. If the rate of change of applied field is extremely slow, then Bean's critical-state model is applicable (provided that there is strong pinning in the superconductor) and one need not solve the magnetic-diffusion equation.

\section{References}

1. Y. Kusano, T. Namba, T. Takada, T. Egi, Y. Ikeda, and M. Takano, Physica C 219 (1994) 366.

2. R. J. Moon, K. P. Trumble, and K. J. Bowman, J. Mater. Res. 14 (1999) 252.

3. P. Majewski, Supercond. Sci. Technol. 10 (1997) 453.

4. W. Zhu and P. S. Nicholsen, J. Appl. Phys. 73 (1993) 8423.

5. M. Tetenbaum, M. Hash, B. S. Tani, J. S. Luo, and V. A. Maroni, Physica C 249 (1995) 396.

6. J. L. MacManus-Driscoll, J. C. Bravman, R. J. Savoy, G. Gorman, and R. B. Beyers, J. Am. Ceram. Soc. 77 (1994) 2305.

7. M. Daumling, R. Maad, A. Jeremie, and R. Flükiger, J. Mater Res. 12 (1997) 1445.

8. N. M. Murphy and S. E. Dorris, work in progress.

\section{Patents, 1998-1999}

Engineered flux pinning centers in BSCCO, TBCCO and YBCO superconductors Kenneth C. Goretta, Michael T. Lanagan, Jieguang Hu, Dean J. Miller, Suvankar Sengupta, John C. Parker, Uthamalingam Balachandran, Donglu Shi, and Richard W. Siegel U. S. Patent 5,929,001 (July 27, 1999).

Method and etchant to join Ag-clad BSCCO superconducting tape Uthamalingam Balachandran, A. N. Iyer, and J. Y. Huang

U.S. Patent 5,882,536 (March 16, 1999).

Elongated Bi-based superconductors made by freeze dried conducting powders Uthamalingam Balachandran, Milan Lelovic, and Nicholas G. Eror

U.S. Patent 5,874,384 (February 23, 1999).

Thin film seeds for melt processing textured superconductors for practical applications Boyd W. Veal, Arvydas Paulikas, Uthamalingam Balachandran, and Wei Zhong U.S. Patent 5,869,431 (February 9, 1999).

Superconductor composite

Stephen E. Dorris, Dominick A. Burlone, and Carol W. Morgan

U.S. Patent 5,866,515 (February 2,1999). 
Surface texturing of superconductors by controlled oxygen pressure

Nan Chen, Kenneth C. Goretta, and Stephen E. Dorris

U.S. Patent 5,856,277 (January 5, 1999).

Method for synthesizing and sinter-forging $\mathrm{Bi}-\mathrm{Sr}-\mathrm{Ca}-\mathrm{Cu}-\mathrm{O}$ superconducting bars

Nan Chen, Kenneth C. Goretta, and Michael T. Lanagan

U.S. Patent 5,821,201 (Oct. 13, 1998).

Mixed-mu superconducting bearings

John R. Hull and Thomas M. Mulcahy

U.S. Patent 5,722,303 (March 3, 1998).

\section{Publications, 1998-1999}

U. Balachandran, Recent Advances in Fabrication of High- $\mathrm{T}_{\mathrm{c}}$ Superconductors for Electric Power Applications, Invited paper presented at NATO Advanced Research Intl. Workshop on High-Temperature Superconductors and Novel Inorganic Materials Engr., Moscow, March 24-29, 1998; in High-Temperature Superconductors and Novel Inorganic Materials, pp. 1-9, 1999, eds. G. Van Tendeloo et al.

U. Balachandran, M. Lelovic, T. Deis, N. G. Eror (U. of Pittsburgh); P. Haldar, and V. Selvamanickam (Intermagnetics General Corp.), Recent Advances in Processing of Ag-Clad Bi-2223 Superconductors, Proc. Cryogenic Engr. Conf. and Intl. Cryogenic Matls. Conf. (CEC/ICMC), Portland, OR, July 28-Aug. 1, 1997; in Advances in Cryogenic Engineering Materials, Vol. 44B, pp. 525-531 (1999).

U. Balachandran; M. Lelovic, B. C. Prorok; N. G. Eror (U. of Pittsburgh); V. Selvamanickam and P. Haldar (Intermagnetics General Corp.), Advances in Fabrication of Ag-Clad Bi-2223 Superconductors, IEEE Trans. Appl. Supercond. 9 (1999) 2474-2479.

U. Balachandran and M. Lelovic; N. G. Eror (U. of Pittsburgh); J. Talvacchio, R. Young (Northrop Grumman); V. Selvamanickam and P. Haldar (IGC), Enhancement of Critical Currents in $(\mathrm{Bi}, \mathrm{Pb})_{2} \mathrm{Sr}_{2} \mathrm{Ca}_{2} \mathrm{Cu}_{3} \mathrm{O}_{\mathrm{y}}(\mathrm{Bi}-2223)$ Superconducting Tapes, Proc. 11 th Intl. Symp. on Superconductivity (ISS '98), Advances in Superconductivity XI, eds. N. Koshizuka and S. Tajima, pp. 947-950 (1999).

Y. S. Cha, D. J. Evans, and J. R. Hull, Measurement of Critical Current and Transient Characteristics of a High-Temperature Superconductor Tube with a Pulsed Current Supply, IEEE Trans. Appl. Supercond. 9 (1999) 1320-1323.

M. P. Chudzik, R. A. Erck, M. T. Lanagan; and C. R. Kannewurf (Northwestern U.), Processing Dependence of Biaxial Texture in Yttria-Stabilized Zirconia by Ion-Beam-Assisted Deposition, IEEE Trans. Appl. Supercond. 9 (1999) 1490-1493.

T. A. Deis, M. Lelovic, N. G. Eror (U. of Pittsburgh); and U. Balachandran, Effect of Ag Doping on Structure and Critical Temperature of $\mathrm{Bi}_{2} \mathrm{Sr}_{2} \mathrm{CaCu}_{2} \mathrm{O}_{8+\delta}$ Superconductors, Appl. Supercond. 6, 279-284 (1998). 
M. B. Field, T. A. Byrne, and D. J. Miller, The Use of Cubic Nd-Ba-Cu-O Seeds to Create $\theta[100], 90^{\circ}-\theta$ [100], and $\theta$ [001] Tilt Y-Ba-Cu-O Grain Boundaries, IEEE Trans. Appl. Supercond. 9 (1999) 1628-1621.

D. K. Finnemore (Ames Laboratory); K. E. Gray; M. P. Maley (Los Alamos National Laboratory); D. O. Welch (Brookhaven National Laboratory); D. K. Christen (Oak Ridge Laboratory) and D. M. Kroeger (Oak Ridge National Laboratory), Coated Conductor Development: An Assessment, Physica C 320 (1999) 1-8.

L. M. Fisher, A. V. Kalinov, S. E. Savel'ev, I. F. Voloshin (All-Russian Electrical Engr. Inst.); P. Haldar (IGC); and U. Balachandran, Losses in Bi-2223/Ag Tapes and in the 1-kA AC Transmission Line Model, Supercond. Sci. Technol. 12 (1999) 24-35.

L. M. Fisher, A. V. Kalinov, S. E. Savel'ev, and I. F. Voloshin (All-Russian Electrical Engr. Inst.); P. Haldar (Intermagnetics General Corp.); H. W. Myron and U. Balachandran, AC Losses in Bi-2223 Tapes and in the 1-kA Transmission Line Model, IEEE Trans. Appl. Supercond. 9 (1999) 1265-1268.

K. C. Goretta, M. M. Cuber, L. R. Feng, B. L. Fisher, M. Jiang, M. T. Lanagan, and U. Balachandran; Y. Xu (Mankato State U.); and M. Xu (U. of Chicago), Microstructure and Properties of $\mathrm{Bi}-\mathrm{Sr}-\mathrm{Ca}-\mathrm{Cu}-\mathrm{O}$ with Additions of Nanometer-Scale Alumina, IEEE Trans. Appl. Supercond. 9 (1999) 1896-1899.

K. C. Goretta, P. Diko, M. Jiang, and M. M. Cuber; M. Xu (U. of Chicago); J. E. Ostenson (Ames Lab.); and S. Sengupta (Superconductive Components, Inc.), Annealing and Mechanical Properties of Bulk Y-Ba-Cu-O, IEEE Trans. Appl. Supercond. 9 (1999) 2081-2084.

K. C. Goretta, D. S. Kupperman, S. Majumdar, M. W. Such; and N. Murayama (Natl. Industrial Res. Inst. of Nagoya, Japan), Elastic Constants of Dense, Textured $(\mathrm{Bi}, \mathrm{Pb})_{2} \mathrm{Sr}_{2} \mathrm{Ca}_{2} \mathrm{Cu}_{3} \mathrm{O}_{\mathrm{y}}$, Supercond. Sci. Technol. 11, 1409-1411 (1998).

K. E. Gray, M. B. Field, and D. J. Miller, Explanation of Low Critical Currents in Flat, Bulk versus Meandering, Thin-Film [001] Tilt Bicrystal Grain Boundaries in $\mathrm{YBa}_{2} \mathrm{Cu}_{3} \mathrm{O}_{7}$, Phys. Rev. B 58 (1998) 9543-9548.

K. E. Gray, D. G. Steel, J. D. Hettinger; and M. M. Eddy (Conductus), Modeling Flux-Flow Dissipation from Randomly Placed Strong-Pinning Sites and Comparison with Ion-Irradiated Cuprate Superconductors, Phys. Rev. B 57 (1998) 13894-13898.

J. R. Hull, Magnetic Levitation, in Encyclopedia of Electrical and Electronics Engineering, Vol. 11, pp. 740-747, ed. J. G. Webster, John Wiley and Sons, New York (1999).

J. R. Hull and T. M. Mulcahy, Gravimeter Using High-Temperature Superconducting Bearing, IEEE Trans. Appl. Supercond. 9 (1999) 390-393.

J. R. Hull; S. Sengupta and J. R. Gaines (Superconductive Components, Inc.), Trapped-Flux Internal-Dipole Superconducting Motor/Generator, IEEE Trans. Appl. Supercond. 9 (1999) 1229-1232. 
N. Iyer, S. Salib, C. Vipulanandan, K. Salama (Texas Center for Superconductivity); and U. Balachandran, Phase Development and Critical Current Density of Bi-2223 Tapes Fabricated by Groove Rolling, Supercond. Sci. Technol. 12 (1999) 436-441.

D. H. Kim, K. E. Gray, N. Jukam, D. J. Miller; Y. H. Kim, J. M. Lee, J. H. Park, and T. S. Hahn (Korea Inst. Sci. \& Technol.), Vortex Motion Noise in $\mathrm{YBa}_{2} \mathrm{Cu}_{3} \mathrm{O}_{\mathrm{x}}$ Films Before and After Heavy-Ion Irradiation, Phys. Rev. B 60 (1999) 3551-3558.

D. H. Kim, J. H. Park, Y. H. Kim, J. M. Lee, T. S. Hahn (Korea Inst. Sci. \& Technol.); J. D. Hettinger and K. E. Gray, Angular Dependence of Hall Conductivity in $\mathrm{YBa}_{2} \mathrm{Cu}_{3} \mathrm{O}_{\mathrm{x}}$ Films with Columnar Defects, Phys. Rev. B 58 (1998) 14215-14218.

D. H. Kim (Yeungnam U., Korea); J. H. Park, Y. H. Kim, J. M. Lee, T. S. Hahn (Korea Inst. of Sci. \& Technol.); J. D. Hettinger, D. J. Miller, and K. E. Gray, Angular Dependence of the Hall Conductivity in $\mathrm{YBa}_{2} \mathrm{Ba}_{2} \mathrm{Cu}_{3} \mathrm{O}_{\mathrm{x}}$ Films with Columnar Defects, Phys. Rev. B 58 (1998) 14215-14218.

J. T. Kim (Korea Res. Inst. of Standards and Science); Y. K. Park, J. C. Park (Korea Res. Inst. of Standards and Science); H. R. Lim, S. Y. Shim, D. H. Kim, W. N. Kang, J. H. Park, T. S. Hahn, S. S. Choi, W. C. Lee (Korea Inst. of Sci. \& Technol.); J. D. Hettinger and K. E. Gray, Pinning Effect on Fluctuation Conductivity in a Superconducting Untwinned $\mathrm{YBa}_{2} \mathrm{Cu}_{3} \mathrm{O}_{\mathrm{x}}$ Single Crystal with Columnar Defects, Physica C 301 (1998) 99-103.

J.-T. Kim, Y. K. Park, J.-C. Park (Korea Res. Inst. of Standards and Science); W. N. Kang, C. W. Chu (Texas Ctr. for Superconductivity at U. of Houston); H. R. Lim (Yeungnam, U., Korea); D. H. Kim (Yeungnam, U., Korea); J. U. Lee, and K. E. Gray, Pinning Effect on Critical Dynamics in $\mathrm{Tl}_{2} \mathrm{Ba}_{2} \mathrm{CaCu}_{2} \mathrm{O}_{8}$ Films Before and After Introducing Columnar Defects, IEEE Trans. Appl. Supercond. 9 (1999) 2296-2299.

M. T. Lanagan, K. C. Goretta, D. K. Walter, and R. B. Poeppel; R. Troendly, M. J. McNallan, and S. Danyluk (Superconducting Products Co.), Near-Net-Shape Fabrication of Continuous Ag-Clad Bi-Based Superconductors, IEEE Trans. Appl. Supercond. 9 (1999) 1900-1903.

D. C. Larbalestier, W. A. Anderson, S. E. Babcock, X. Y. Cai (Appl. Superconductivity Ctr.); S. E. Dorris; M. Feldman, J. Jiang (Appl. Superconductivity Ctr.); Q. Li (Am. Superconductor Corp.); J. A. Parrell (Oxford Superconducting Tech.); R. Parrella (Amer. Superconductor Corp.); M. Polak (Slovak Academy of Sciences); A. Polyanskii (Appl. Superconductivity Ctr.); G. N. Riley Jr., M. Rupich (Am. Superconductor Corp.); and Y. Wu (Appl. Superconductivity Ctr.), New Experiments Elucidating the Current Limiting Mechanisms of Ag-Sheathed $(\mathrm{Bi}, \mathrm{Pb})_{2} \mathrm{SrCa}_{2} \mathrm{Cu}_{3} \mathrm{O}$ Tapes, Proc. 11 th Intl. Symp. on Superconductivity (ISS '98), Fukuoka, Japan, Nov. 16-19, 1998; in Advances in Superconductivity XI, eds. N. Koshizuka and S. Tajima, pp. 805-810.

M. Lelovic, N. G. Eror (U. of Pittsburgh); U. Balachandran, B. C. Prorok; V. Selvamanickam, P. Haldar (IGC); J. Talvacchio and R. Young (Northrup Grumman), Shielded High-T $(\mathrm{Bi}, \mathrm{Pb})_{2} \mathrm{Sr}_{2} \mathrm{Ca}_{2} \mathrm{Cu} 3 \mathrm{O}_{\mathrm{y}}(\mathrm{Bi}-2223)$ Superconducting Tapes, Supercond. Sci. Technol. 11, 1255-1260 (1998). 
M. Lelovic, S. Mench, T. Deis, N. G. Eror (U. of Pittsburgh); U. Balachandran; V. Selvaminickam and P. Haldar (IGC), AC Magnetic Field Losses in BSCCO-2223 Superconducting Tapes, Proc. Cryogenic Engineering Conf. and Intl. Cryogenic Matls. Conf. (CEC/ICMC) Portland, OR, July 28-Aug. 1, 1997; in Advances in Cryogenic Engineering Materials, Vol. 44B, pp. 715-722 (1999).

V. A. Maroni, A. K. Fischer, and K. T. Wu, Using Imagining Raman Spectroscopy to Explore Phase Evolution in Composite Ceramic Superconductors, in "Spectroscopy of Superconducting Materials," ACS Symp. Series 730, ed. E. Faulques (Am. Chem. Soc., Washington, DC, 1999) pp. 156-167.

V. A. Maroni; M. Teplitsky and M. W. Rupich (American Superconductor Corp.), An environmental scanning electron microscope study of the Ag/Bi-2223 composite conductor from 25 to $840^{\circ} \mathrm{C}$, Physica C 313 (1999) 169-174.

N. N. Merchant, J. S. Luo, A. K. Fischer, V. A. Maroni; E. R. Podtburg, W. L. Carter, Q. Li, A. Otto, M. W. Rupich, and G. N. Riley Jr. (American Superconductor Corp.), Phase Formation and Microstructure Development in Silver-Clad Bi-2223 Multifilament Composite Conductors, Supercond. Sci. Technol. 12 (1999) 327-336.

N. N. Merchant, D. J. Miller, V. A. Maroni; R. D. Parrella, Q. Li, M. W. Rupich, W. L. Carter, and G. N. Riley Jr. (American Suyperconductor Corp.), Phase Stability and Grain Growth in an Ag/Bi-2223 Composite Conductor Prepared Using Fine-Grained Bi-2223 as a Precursor, IEEE Trans. Appl. Supercond. 9 (1999) 2545-2548.

D. J. Miller, K. E. Gray, M. B. Field; and D. H. Kim (Yeungnam U., Korea), The Influence of Vortex Pinning and Grain Boundary Structure on Critical Currents Across Grain Boundaries in $\mathrm{YBa}_{2} \mathrm{Cu}_{3} \mathrm{O}_{\mathrm{x}}$, IEEE Trans. Appl. Supercond. 9 (1999) 2030-2033.

D. J. Miller, V. R. Todt, M. St. Louis-Weber, X. F. Zhang, D. G. Steel, M. B. Field, and K. E. Gray, Microstructure and Transport Behavior of Grain Boundaries in $\mathrm{YBa}_{2} \mathrm{Cu}_{3} \mathrm{O}_{\mathrm{y}}$, A Comparison Between Thin Films and Bulk Bicrystals, Mater. Sci. Eng. B53 (1998) 125-131.

J.-H. Park, K. C. Goretta; and N. Murayama (Natl. Industrial Res. Inst. of Nagoya, Japan), Chemical Diffusion of Oxygen in Dense, Textured $(\mathrm{Bi}, \mathrm{Pb})_{2} \mathrm{Sr}_{2} \mathrm{Ca}_{2} \mathrm{Cu}_{3} \mathrm{O}_{\mathrm{x}}$, Physica C 312 (1999) 269-273.

S. Salib, A. N. Iyer, C. Vipulanandan, K. Salama (Texas Center for Superconductivity at U. of Texas, Houston); and U. Balachandran, Electromechanical Characterization of Silver-Clad BSCCO Tapes, Appl. Supercond. 6 (1998) 1-9.

S. Vikram, P. A. Molian, M. D. Shirk (Iowa State U.); R. E. Koritala and U. Balachandran, Influence of Pulse Width and Target Density on Pulsed Laser Deposition of Thin YBaCuO Films, Proc. 17th Intl. Congress on Applications of Lasers and Electro-Optics, Orlando, Nov. 16-19, 1998; in Laser Materials Processing Vol. 85, pp. 96-105. 
Y. Xu (Mankato State U.); K. C. Goretta, M. M. Cuber, M. L. Burdt, L. R. Feng, N. Chen, U. Balachandran; and M. Xu (U. of Chicago), Processing Considerations for Adding Nanometer-Scale Oxides to Enhance Flux Pinning in High-Temperature Superconductors, Proc. Cryogenic Engineering Conference/Intl. Cryogenic Materials Conf. (CEC/ICMC), Portland, OR, July 28-Aug. 1, 1997; in Advances in Cryogenic Engineering Materials, Vol. 44B, pp. 493-500 (1999).

Z. J. Yang, Interaction between a Magnetic Dipole and a Superconducting Sphere, Solid State Commun. 107 (1998) 745-749.

Z. J. Yang, J. R. Hull; J. A. Lockwood and T. D. Rossing (Northern Illinois U.), Forces and Torques between Two Square Current Loops, Intl. J. Appl. Electromagn. Mech. 10 (1999) 63-76.

A. H. Zheng, M. Jiang, Yi Huang, B. W. Veal, and H. Claus, Growth and Physical Properties of Melt Textured (Gd,Y)Ba ${ }_{2} \mathrm{Cu}_{3} \mathrm{O}_{\mathrm{x}}$, Physica C 307 (1998) 284-290. 
Distribution for ANL-99/20

Internal:

T. Askew

K. E. Gray

Q. $\mathrm{Hu}$

U. Balachandran

J. R. Hull

T. Mulcahy

Y. S. Cha

J. Jorgensen

J. J. Picciolo

G. Crabtree

M. Kirk

R. B. Poeppel

S. E. Dorris

S. Lake

A. C. Raptis

H. Drucker

M. Lelovic

W. J. Shack

J. E. Emerson

B. Ma

W. W. Schertz

B. L. Fisher

C. A. Malefyt

F. Y. Fradin

V. A. Maroni

J. P. Singh

J. M. Gibson

D. J. Miller

K. L. Uherka

A. M. Wolsky

K. C. Goretta

TIS Files

External:

DOE-OSTI (2)

ANL-E Library

ANL-W Library

\section{U.S. Department of Energy, Washington:}

Office of Power Technologies, Energy Efficiency and Renewable Energy:

B. Brewer

J. Daley

R. K. Dixon

R. George

Basic Energy Sciences-Materials Science:

T. J. Fitzsimmons

R. Gottschall

B. Strauss

Energy Technology Division Review Committee:

H. K. Birnbaum, University of Illinois at Urbana-Champaign

I.-Wei Chen, University of Pennsylvania

E. M. Logothetis, Ford Motor Co., Dearborn, MI

H. S. Rosenbaum, Fremont, CA

S. L. Sass, Cornell University

R. K. Shah, General Motors Corp., Lockport, NY

S. Smialowska, Ohio State University, Columbus

Other - Industry - University

R. G. Abboud, Commonwealth Edison, Zion, IL

S. Ahmed, Southern California Edison, Rosemead, CA

J. Badin, Energetics, Inc., Columbia, MD

A. L. Bement, Jr., Midwest Superconductivity Consortium, Purdue University

R. Blaugher, National Renewable Energy Laboratory 
R. W. Boom, University of Wisconsin - Madison

D. Burlone, BASF Corp., Enka, NC

J. Cave, Hydro Quebec, Montreal, Canada

F. Chu, Ontario HydroTechnology, Toronto, Canada

P. Chu, University of Houston

J. Clem, Ames Laboratory

B. Currie, Pacific Northwest National Laboratory

A. Day, Boeing Corp., Seattle, WA

D. Driscoll, Rockwell Automation, Cleveland, $\mathrm{OH}$

M. Eddy, Superconductor Technologies, Inc., Santa Barbara, CA

J. Ekin, National Institute of Standards and Technology, Boulder, CO

N. Eror, University of Pittsburgh

D. K. Finnemore, Ames Laboratory

M. Fluss, Lawrence Livermore National Laboratory

S. Foner, Francis Bitter National Magnet Lab., Massachusetts Institute of Technology

A. Funkenbusch, 3M, St. Paul, MN

J. R. Gaines, Jr., Superconductive Components, Inc., Columbus, OH

P. Grant, EPRI, Palo Alto, CA

T. Grudkowski, United Technologies Research Center, East Hartford, CT

D. Gubser, Naval Research Laboratory

P. Haldar, Intermagnetics General Corp., Latham, NY

R. A. Hawsey, Oak Ridge National Laboratory

Z. Haq, Energetics, Inc., Washington, DC

R. L. Hughey, Southwire Co., Carrollton, GA

J. B. Ketterson, Northwestern University

M. Klein, University of Illinois at Urbana-Champaign

S. F. Kral, Babcock \& Wilcox, Lynchburg, VA

D. K. Kroeger, Oak Ridge National Laboratory

A. Lamanna, Energetics Inc., Washington, DC

D. C. Larbalestier, University of Wisconsin - Madison

A. Lauder, DuPont, Wilmington, DE

E. Leung, General Atomics, San Diego, CA

M. Levy, University of Wisconsin - Milwaukee

J. Lewis, University of Illinois at Urbana-Champaign

R. Loehman, Sandia National Laboratories

A. Malozemoff, American Superconductor Corp., Westborough, MA

F. Marciano, Pirelli Cables, Columbia, SC

K. Marken, Oxford Instruments, Inc., Carteret, NJ

B. McCallum, Ames Laboratory, Ames, IA

P. J. McGinn, University of Notre Dame

S. Mehta, Waukesha Electric Systems, Waukesha, WI

D. Moon, Westinghouse, Pittsburgh, PA

F. C. Moon, Cornell University

M. W. Morgan, Ability Engineering Technology, Inc., South Holland, IL

L. Motowidlo, IGC Advanced Superconductors, Inc., Waterbury, CT

J. Muehlhauser, University of Tennessee

C. Oberly, Wright Air Force Laboratory, Dayton, $\mathrm{OH}$

D. Peterson, Los Alamos National Laboratory

N. Phillips, E. O. Lawrence Berkeley National Laboratory 
C. Rey, DuPont, Wilmington, DE

R. A. Roehl, Commonwealth Edison Co., Downers Grove, IL

C. H. Rosner, Intermagnetics General Corp., Latham, NY

R. Schiferl, Reliance Electric, Cleveland, $\mathrm{OH}$

J. Schwartz, Florida State University

U. Sinha, Southwire Co., Carrollton, GA

G. Smith, PSC, Inc., Woodinville, WA

M. Strasik, Boeing Corp., Seattle

M. Suenaga, Brookhaven National Laboratory

P. Tsivitse, Hunting Valley, $\mathrm{OH}$

J. Voight, Sandia National Laboratories

B. Weinberger, United Technologies Research Center, East Hartford, CT

D. O. Welch, Brookhaven National Laboratory

J. Worth, Oxford Instruments, Inc., Carteret, NJ

J. Wu, Westinghouse Electric Corp., Science and Technology Center, Pittsburgh

G. O. Zimmerman, ZerRes Corp., Boston 